TOPICAL REVIEW

\title{
Solving the three-body Coulomb breakup problem using exterior complex scaling
}

\author{
C W. McCurdy ${ }^{1,2}$ M. Baertschy ${ }^{3}$ and T. N Rescigno ${ }^{1}$ \\ ${ }^{1}$ Lawrence Berkeley National Laboratory, Chemical Sciences, Berkeley, CA \\ 94720 \\ ${ }^{2}$ Department of Applied Science, University of California, Davis, CA 95616 \\ ${ }^{3}$ Colorado University, Denver, CO 80217 \\ E-mail: cwmccurdy@lbl.gov, mark@physics.cudenver.edu tnrescigno@lbl.gov
}

\begin{abstract}
Electron impact ionization of the hydrogen atom is the prototypical three-body Coulomb breakup problem in quantum mechanics. The combination of subtle correlation effects and the difficult boundary conditions required to describe two electrons in the continuum have made this one of the outstanding challenges of atomic physics. A complete solution of this problem in the form of a "reduction to computation" of all aspects of the physics is given by the application of exterior complex scaling, a modern variant of the mathematical tool of analytic continuation of the electronic coordinates into the complex plane that was used historically to establish the formal analytic properties of the scattering matrix.

This review first discusses the essential difficulties of the three-body Coulomb breakup problem in quantum mechanics. It then describes the formal basis of exterior complex scaling of electronic coordinates as well as the details of its numerical implementation using a variety of methods including finite difference, finite elements, discrete variable representations, and B-splines. Given these numerical implementations of exterior complex scaling, the scattering wave function can be generated with arbitrary accuracy on any finite volume in the space of electronic coordinates, but there remains the fundamental problem of extracting the breakup amplitudes from it. Methods are described for evaluating these amplitudes. The question of the volume-dependent overall phase that appears in the formal theory of ionization is resolved. A summary is presented of accurate results that have been obtained for the case of electron-impact ionization of hydrogen as well as a discussion of applications to the double photoionization of helium.
\end{abstract}

\section{Contents}

1 Introduction 2

1.1 Formal theory of ionization . . . . . . . . . . . . . 3

1.2 Practical approaches to ionization $\ldots \ldots \ldots \ldots \ldots$

1.3 Approximate two-body boundary conditions . . . . . . . . . . 5

2 Complex exterior scaling $\quad 7$

2.1 The origins of complex coordinate scaling . . . . . . . . 7

2.2 Formulation of complex scaling for arbitrary complex coordinate contours 9

2.3 "Sharp" Exterior scaling and the question of the discontinuity of the Jacobian . . . . . . . . . . . . . . . . . . . . 11

2.4 Making complex scaling work for long range potentials . . . . . . . 11 
3 Numerical implementations of exterior complex scaling $\quad 14$

3.1 Finite differences . . . . . . . . . . . . . . . . . 15

3.2 Finite Elements and the Discrete Variable Representation . . . . . . 16

3.3 B-splines . . . . . . . . . . . . . . . . . . . . . . . . . . . 21

3.4 Direct numerical integration . . . . . . . . . . . . . . . 24

4 Calculating amplitudes and cross sections $\quad 25$

4.1 Amplitudes for breakup by electron impact . . . . . . . . . . . 26

4.2 Amplitudes for inelastic scattering . . . . . . . . . . . . . . . 28

4.3 Amplitudes for double photoionization . . . . . . . . . . . . . 29

5 Partial wave analysis of breakup amplitudes $\quad \mathbf{3 0}$

5.1 Electron-impact ionization . . . . . . . . . . . . . . . . 30

5.2 Double photoionization $\ldots \ldots \ldots \ldots \ldots \ldots \ldots \ldots$

6 The overall phase of the breakup amplitude in the formal theory of ionization

7 Accurate amplitudes for electron impact ionization of the hydrogen $\begin{array}{ll}\text { atom } & \mathbf{3 7}\end{array}$

7.1 Numerical methods . . . . . . . . . . . . . . . . . . . . 37

7.2 Single and double differential cross sections . . . . . . . . . . . 38

7.3 Triple differential cross sections . . . . . . . . . . . . . . . . 40

8 Double photoionization of the helium atom 45

8.1 Numerical solution of the first-order wave equation . . . . . . . . . 45

8.2 Single and triple differential cross sections . . . . . . . . . . . 46

9 Conclusion and outlook

\section{Introduction}

Electron-impact ionization of an atom is one of the most basic, and important, problems in atomic collision physics. From a theoretical viewpoint, it is also one of the most difficult. Indeed, it is only within the past few years that the simplest Coulomb three-body breakup problem - electron-hydrogen atom ionization - has been reduced to practical computation in the sense of having a formalism and the associated numerical algorithms that allow the calculation of the relevant physical quantities to any desired accuracy. The central difficulty that impeded progress on the problem of three-body breakup in Coulomb systems (both collisional breakup or "e,2e" and double photoionization or " $\gamma, 2 \mathrm{e}$ ") is the cumbersome asymptotic form of the wave function that emerges from a formal treatment of the problem. How that formal difficulty was circumvented is the subject of this review. Before we describe that development, however, it is useful to begin with a short summary of the formal theory, along with a brief description of other theoretical approaches to the problem, all of which helped to motivate the methods that form the main subject of this review. 


\subsection{Formal theory of ionization}

While perturbative treatments of the problem have been used since the early days of quantum mechanics, a formal theory was not developed until the 1960s, when Peterkop [1] and Rudge and Seaton [2,3] independently deduced the appropriate boundary conditions for electron-hydrogen atom ionization to be:

$$
\Psi^{+}\left(\mathbf{r}_{1}, \mathbf{r}_{2}\right)=\Psi_{0}\left(\mathbf{r}_{1}, \mathbf{r}_{2}\right)+\Psi^{(s c)+}\left(\mathbf{r}_{1}, \mathbf{r}_{2}\right)
$$

with

$\Psi^{(s c)+}\left(\mathbf{r}_{1}, \mathbf{r}_{2}\right) \underset{\rho \rightarrow \infty}{\longrightarrow}-f\left(\hat{r}_{1}, \hat{r}_{2}, \alpha\right) \sqrt{\frac{i \kappa^{3}}{\rho^{5}}} \exp i\left(\kappa \rho+\frac{\zeta\left(\hat{r}_{1} \cdot \hat{r}_{2}, \alpha\right)}{\kappa} \ln (2 \kappa \rho)\right)$

where $\Psi_{0}\left(\mathbf{r}_{1}, \mathbf{r}_{2}\right)$ is the unperturbed wave function describing the initial state, $f$ is the ionization amplitude and the hyperspherical coordinates are defined by $\rho=\left(r_{1}^{2}+r_{2}^{2}\right)^{1 / 2}$ with $\alpha=\tan ^{-1}\left(r_{1} / r_{2}\right)$, and $\kappa$ is related to the total energy by $E=\kappa^{2} / 2$.

Knowledge of this asymptotic form, however, has yet to provide a practical path for numerical computation. Firstly, Eq.(2) is only valid in the far asymptotic region and only when all three charged particles are well separated. Moreover, the coefficient $\zeta\left(\hat{r}_{1} \cdot \hat{r}_{2}, \alpha\right)$ of the logarithmic phase depends on the distances as well as the angle between the two ejected electrons. The most severe problem, however, is the fact that Eq.(2) is not separable in spherical coordinates, and is therefore much more cumbersome to apply to numerical calculations which are perforce done in that coordinate system. As a consequence, no one has yet applied Eq.(2) to the numerical solution of the Schrödinger equation for the ionization problem.

The formal theory of Peterkop and of Rudge and Seaton poses another challenge to computation. The ordinary expression for evaluating the amplitude, starting from the scattering wave function that solves the Schrödinger equation, does not apply, because defined in the usual way it would have an infinite phase associated with integrating an expression with logarithmic phases over an infinite volume. Instead the ionization amplitude for producing electrons with momenta $\mathbf{k}_{1}$ and $\mathbf{k}_{2}$ is given by $[1,2,3]$

$f\left(\mathbf{k}_{1}, \mathbf{k}_{2}\right)=-(2 \pi)^{5 / 2} e^{i \Delta\left(\mathbf{k}_{1}, \mathbf{k}_{2}\right)} \iint \Psi^{+}(H-E) \phi\left(-\mathbf{k}_{1}, z_{1}\right) \phi\left(-\mathbf{k}_{2}, z_{2}\right) d \mathbf{r}_{1} d \mathbf{r}_{2}$,

with effective charges in the one-body Coulomb functions, $\phi(-\mathbf{k}, z)$ depending on both the energy and direction of ejection of each electron, chosen to satisfy the so-called Peterkop condition,

$$
\frac{z_{1}}{k_{1}}+\frac{z_{2}}{k_{2}}=\frac{1}{k_{1}}+\frac{1}{k_{2}}-\frac{1}{\left|\mathbf{k}_{1}-\mathbf{k}_{2}\right|}
$$

with

$$
\Delta\left(\mathbf{k}_{1}, \mathbf{k}_{2}\right)=2\left[\left(z_{1} / k_{1}\right) \ln \left(k_{1} / \kappa\right)+\left(z_{2} / k_{2}\right) \ln \left(k_{2} / \kappa\right)\right] .
$$

A formal theory of ionization can also be based on the so-called plane-wave form of the wave function, $\Psi_{k_{1}, k_{2}}^{-}$, in which the initial channel is one where both electrons are in the continuum. The leading term in the asymptotic expansion of $\Psi_{k_{1}, k_{2}}^{-}$, again for the case where all particles are well separated, was first obtained by Redmond [4]:

$$
\begin{aligned}
\Psi_{k_{1}, k_{2}}^{-}\left(\mathbf{r}_{1}, \mathbf{r}_{2}\right) \sim & e^{i\left(\mathbf{k}_{1} \cdot \mathbf{r}_{1}+\mathbf{k}_{2} \cdot \mathbf{r}_{2}\right)} \\
& \times e^{i / k_{1} \ln \left(k_{1} r_{1}+\mathbf{k}_{1} \cdot \mathbf{r}_{1}\right)} e^{i / k_{2} \ln \left(k_{2} r_{1}+\mathbf{k}_{2} \cdot \mathbf{r}_{2}\right)} \\
& \times e^{\left.-i /\left|\mathbf{k}_{1}-\mathbf{k}_{2}\right| \ln \left(\left|\mathbf{k}_{1}-\mathbf{k}_{2}\right|\left|\mathbf{r}_{1}-\mathbf{r}_{2}\right| / 2\right)+\left(\mathbf{k}_{1}-\mathbf{k}_{2}\right) \cdot\left(\mathbf{r}_{1}-\mathbf{r}_{2}\right) / 2\right)} .
\end{aligned}
$$


Brauner, Briggs and Klar [5] subsequently showed that the Redmond wave function is the asymptotic form of what is now generally referred to as the $3 C$ wave function:

$\Psi_{k_{1}, k_{2}}^{-}\left(\mathbf{r}_{1}, \mathbf{r}_{2}\right) \sim e^{i\left(\mathbf{k}_{1} \cdot \mathbf{r}_{1}+\mathbf{k}_{2} \cdot \mathbf{r}_{2}\right)}$

$$
\times C\left(-1 / k_{1}, \mathbf{k}_{1}, \mathbf{r}_{1}\right) C\left(-1 / k_{2}, \mathbf{k}_{2}, \mathbf{r}_{2}\right) C\left(1 / k_{12}, \mathbf{k}_{12} / 2, \mathbf{r}_{12}\right)
$$

where

$$
C(\alpha, \mathbf{k}, \mathbf{r})=\Gamma(1-i \alpha) e^{-\pi \alpha / 2}{ }_{1} F_{1}(i \alpha ; 1 ;-i k r-i \mathbf{k} \cdot \mathbf{r})
$$

The $3 C$ wave function has spawned a series of sophisticated distorted wave calculations, which have been used to study ionization cross sections at intermediate and higher energies, with varying degrees of success. Much of this work has been reviewed by Lucey, Rasch and Whelan [6] and by Jones and Madison [7].

The formal theory of three-body Coulomb breakup continues to be an active area of research. Kim and Zubarev [8], building on the work of Alt and Mukhamedzhanov [9], have shown that the $3 C$ wave function is asymptotically correct in all asymptotic domains, i.e. it is the leading term in the asymptotic expansion of $\Psi_{k_{1}, k_{2}}^{-}$where any two particles are far apart. More recently, Kadyrov et al. [10] have established an integral relationship that connects $\Psi^{+}$to $\Psi_{k_{1}, k_{2}}^{-}$. Since the asymtotically correct behavior of $\Psi_{k_{1}, k_{2}}^{-}$is known in all domains of coordinate space $[9,11]$, they were able to use this relationship to derive the asymptotic behavior of $\Psi^{+}$in regions where the Peterkop wave function Eq.(2) is invalid.

While many of the gaps in the formal theory have been filled, it is clear that we are still far from being able to implement the formal theory in a practical calculation, i.e. from using the asymptotic form of the exact wave function as a boundary condition for solving the time-independent Schrödinger equation. At the very least, a partial-wave decomposition of the full three-body wave function would be required and this has yet to be accomplished.

\subsection{Practical approaches to ionization}

Practical approaches to an ab initio treatment of ionization have either employed approximate ionization boundary conditions or have been designed to circumvent them entirely. Exterior complex scaling, which is the central topic of this review, falls into this latter category. Several other methods can be cited in the same context.

In the hyperspherical $R$-matrix method with semiclassical outgoing waves [12], the time-independent Schrödinger equation is solved without detailed specification of three-body Coulomb bounday conditions by merging two different approaches: an Rmatrix treatment of the two-electron system in the vicinity of the nucleus along with a semiclassical description of the evolution of the system in the asymptotic region. This method has been successfully applied to the $(\gamma, 2 \mathrm{e})$ problem.

There have also been successful computational approaches to the three-body Coulomb problem based on solving the time-dependent Schrödinger equation, such as the "time-dependent close-coupling" approach of Pindzola, Schultz, Robicheaux and coworkers $[13,14]$. In that approach, which has been applied to both $(\mathrm{e}, 2 \mathrm{e})$ and $(\gamma, 2 \mathrm{e})$ problems, a wave packet is fired at the target atom and the time-dependent Schrödinger equation describing its dynamics is solved in a close-coupling formulation. There is thus no ambiguity about the boundary conditions, since the time-dependent Schrödinger equation is solved as an initial value problem. 


\subsection{Approximate two-body boundary conditions}

A broad class of applications have been carried out by employing approximate twobody boundary conditions in solving the time-independent Schrödinger equation above the ionization threshold. Without attempting anything like an exhaustive review, we will briefly describe work on extending the close-coupling (CC) formalism, which for over 40 years has formed the basis for most ab initio work on calculating atomic excitation cross sections in low-energy electron scattering, to treat ionization.

In the close-coupling method, the internal target states are used as a basis for expanding the full wave function. The target-state basis must obviously be truncated in actual computations and early applications were limited to the use of a few spectroscopic states. In the late 1960s, it was learned that convergence of excitation cross sections could be accelerated by including positive-energy "pseudostates" in the expansion [15], typically obtained by diagonalizing the target Hamiltonian in a basis of square-integrable functions. For the close-coupling expansion to "converge", the target states must approach completeness in the interaction region. That this could be achieved in a practical calculation was first demonstrated for the $e-\mathrm{H}$ problem by Bray and Stelbovics [16] who showed that, by systematically increasing the pseudostates in a "convergent close-coupling" (CCC) scheme, the unphysical structures that typically appear in the cross sections near pseudostate thresholds eventually disappear. They also demonstrated that the sum of the excitation cross sections into positive-energy pseudostates gives an accurate representation of the total ionization cross section. Similar results have since been achieved with other close-coupling methods, such as the $R$-matrix method [17].

The success of these methods in computing total ionization cross sections is ultimately tied to the completeness of the underlying basis and the unitarity of the close-coupling formalism. Convergence of the total ionization cross section is guaranteed by convergence of the discrete excitation cross sections, since the former can be obtained by subtracting the latter from the total cross section, which in turn can be obtained using the optical theorem. The computation of differential ionization cross sections, however, is not guaranteed by the underlying formalism and additional assumptions have to be made. In the CCC method, for example, one assumes that the amplitudes for exciting individual positive-energy pseudostates can be equated with a true ionization amplitude by simply renormalizing the pseudostate to the true target continuum function $[18,19]$. When this method was implemented, however, it was found to yield an asymmetric single differential cross sections (SDCS) that oscillated as a function of energy and that did not converge with increasing number of target pseudostates $[20,21]$.

The SDCS, or energy-sharing cross section $(d \sigma / d \epsilon)$, yields the total ionization cross section when integrated over the energy of an ejected electron:

$$
\sigma^{i o n}(E)=\int_{0}^{E} \frac{d \sigma}{d \epsilon}
$$

where $E$ is the energy relative to the ionization threshold. Since the electrons are indistinguishable, the SDCS should be symmetric about $E / 2$. This property does not follow automatically from the close-coupling formalism, even though the underlying wave function is antisymmetric, since the two electrons are treated inequivalently, one through discretization, the other with two-body scattering boundary conditions. It was observed that the SDCS cross sections computed using pseudostate excitation amplitudes tend rapidly to zero when the pseudostate energy is greater than $E / 2$. 
This led Bray [21] to hypothesize that a close-coupling expansion that used an infinite number of pseudostates would produce an SDCS equal to the correct value for $\epsilon<E / 2$ and zero for $\epsilon>E / 2$. If the true SDCS were nonzero at $E / 2$, then the resulting step discontinuity at $E / 2$ would produce the observed oscillatory behavior in finite basis set close-coupling calculations.

Two mathematical studies have attempted to explain the peculiar behavior observed in the SDCS computed in close-coupling calculations. Stelbovics [22] has analyzed the Temkin-Poet (TP) model, which simplifies the full e- $\mathrm{H}$ problem by treating only zero angular momentum states. The Schrödinger equation in this model,

$$
\left(\frac{d^{2}}{d x^{2}}+\frac{d^{2}}{d y^{2}}+\frac{2}{\min (x, y)}+2 E\right) \Psi(x, y)=0,
$$

is separable in the regions $x>y$ and $x<y$, so the exact solution can be constructed from products of $l=0$ free states and hydrogenic states. By comparing the asymptotic behavior of the exact solution with the form used in a close-coupling calculation, he was able to show that the CCC result should converge, but only at the point $E / 2$, to give half the correct amplitude, or one quarter the true cross section, as had been empirically observed [21]. At other energies, the CCC wave function will generally have an asymptotic form that is incompatable with that of the exact wave function. Thus Stelbovics was able to explain Bray's step discontinuity hypothesis for the TemkinPoet case. Stelbovics speculated that the full e-H problem might show the same behavior, but the arguments he employed were specific to the Temkin-Poet model.

Rescigno et al. [23], using stationary phase arguments, were able to extend the demonstration of the step discontinuity behavior to the full three-body breakup problem, but unfortunately only for cases involving short-range potentials. They also showed how a wave function that satisfied the proper asymptotic behavior for breakup would nonetheless yield an anomolous step discontinuity behavior when it was projected onto a set of discrete, box-normalized states and when the breakup cross sections was computed via the CCC prescription. They speculated that, since this behavior was obtained from an asymptotically correct wave function, the anomolous behavior might be the result of the way the ionization cross section was extracted from the calculation rather than a problem with the wave function itself. This idea has since been tested in an $R$-matrix calculation for the Temkin-Poet model by Pindzola, Mitnik and Robicheaux [24]. The step behavior in the SDCS was successfully eliminated and a symmetric cross section was obtained, but the oscillations in the SDCS persisted, indicating that the reformulated procedure for evaluating the SDCS is only a partial remedy for errors introduced by using approximate two-body boundary conditions to generate the wave function.

The empirical evidence that something like the step discontinuity hypothesis pertains to the full problem is strong, despite the absence of a rigorous demonstration of its veracity. The magnitude of the oscillations in the SDCS that are observed for e-H evidently diminish with increasing energy [25] and do not pose a problem above $\sim 55 \mathrm{eV}$. At lower energies, where the oscillations become more pronounced, the true SDCS is relatively flat, so that a knowledge of its value at one point, along with the total cross section, makes it relatively easy to estimate its value at all energy sharings. Alternatively, Scott et al [26], employing the intermediate $R$-matrix method with very large expansion basis sets and very large box sizes, found they could obtain the most accurate values for the $L=0$ component of the full SDCS by energy-averaging the results of a number of $R$-matrix calculations, excluding points near $E / 2$. 
In light of these practical difficulties with CC methods that employ approximate two-body boundary conditions, it is clearly be desirable to find a way around the need for explicit enforcement of asymptotic boundary conditions from the outset. The method of exterior complex scaling does precisely that.

\section{Complex exterior scaling}

\subsection{The origins of complex coordinate scaling}

Although the modern uses of complex coordinates are in numerical computation, the idea was invented for a very different purpose in formal scattering theory. In fact the origins of the idea of complex coordinate scaling in the Schrödinger equation are in the early efforts which established the analytic properties of the $S$-matrix in the complex momentum and energy planes, most notably in the work Regge [27, 28]. The notion of scaling the coordinates of the particles by a complex phase factor,

$$
r \rightarrow r e^{i \eta}
$$

appears, for example, in paper in 1962 by Regge and coworkers [29]. Later the idea was very much generalized in a formal context and extended to systems of particles interacting via Coulomb potentials by Aguilar and Combes [30], Simon [31] and by Balslev and Combes [32]

A central result of that work is the understanding that the spectrum of the Hamiltonian is distorted in a particular way, shown in Fig. 1, that ultimately proves useful in numerical calculations. The continuous spectrum associated with each threshold is rotated downwards in the energy plane by $2 \eta$, discrete resonance eigenvalues are revealed, and the bound state energies are unchanged. The disclosure of the resonance eigenvalues arises because under complex scaling, their pure outgoing form, $\psi_{\text {res }}(r) \underset{r \rightarrow \infty}{\longrightarrow} \exp \left(i k_{r e s} r\right)$, becomes exponentially decaying, $\exp \left(i\left|k_{r e s}\right| e^{-i \alpha} r e^{i \eta}\right) \underset{r \rightarrow \infty}{\longrightarrow} 0$, if the scaling angle, $\eta$ is large enough that $\eta-\alpha>0$, where $\alpha$ is the argument of $k_{\text {res }}$. Therefore, under complex scaling the resonance wave function can be rendered square integrable. Following the pioneering work in 1974 of Doolen, Nuttal and Stagat [33], this fact led to the widespread use of complex scaling in direct calculations of resonance energies of various kinds, in a literature that has been reviewed elsewhere [34], and continues, for example, with the highly precise calculations of Ho [35, 36].

However it was the earlier work Nuttall and Cohen [37] in 1969 that extended these ideas to the direct computation of scattering amplitudes, and did so first in the context of three-body problems above the breakup threshold. Unfortunately, complex scaling could only be applied by those original methods to the calculation of scattering amplitudes for short-range potentials (finite range or decaying at least exponentially as the coordinates increase). That remained the state of the art for decades after, until modern applications of the idea of exterior complex scaling that we will discuss in this topical review.

In the context of the direct calculation of resonance energies, another problem arose in the first applications to molecular systems. The problem was that, in the Born-Oppenheimer approximation, the nuclear attraction potentials were not analytic under the original complex scaling transformation in Eq.(11). To avoid that problem Simon [38] suggested making an exterior complex scaling (ECS) transformation on 
(a)

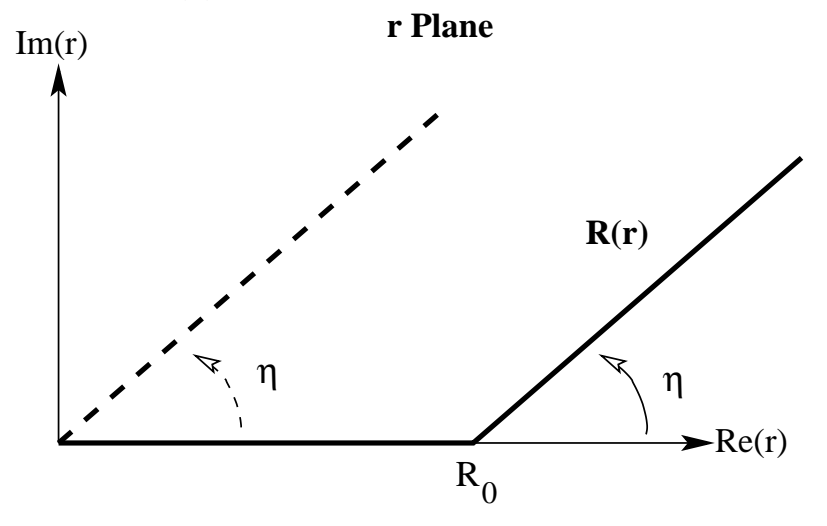

(b)

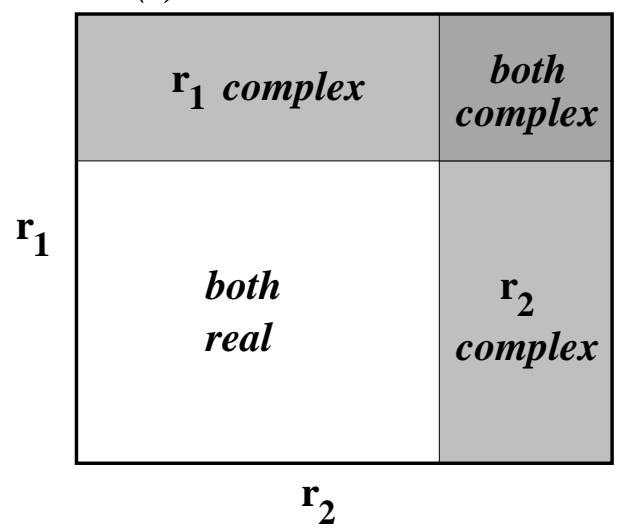

(c)

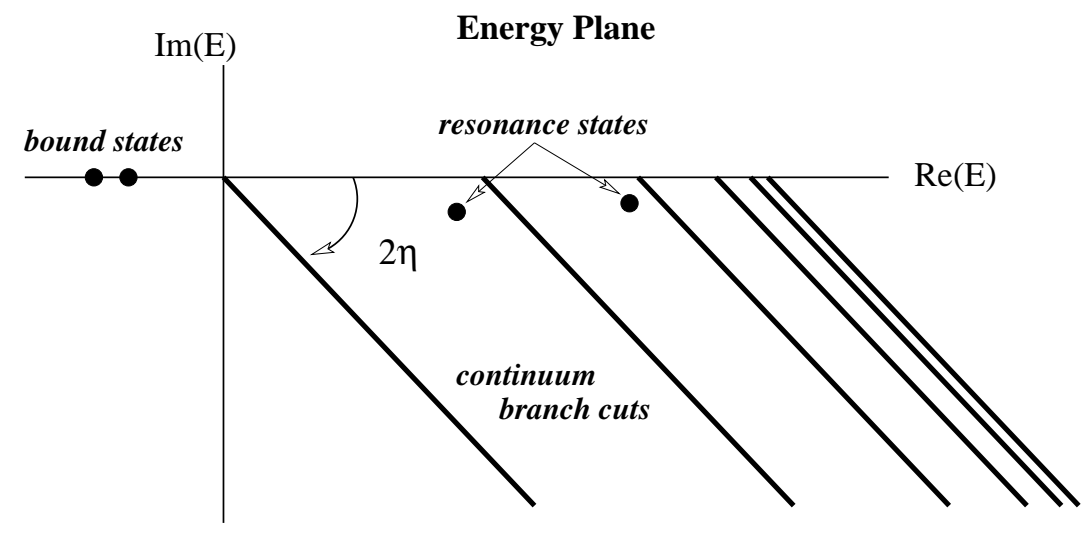

Figure 1. Exterior complex scaling: (a) Simple complex scaling (rotated coordinates) and exterior complex scaling contours in the complex $\mathrm{r}$ plane, (b) Application of exterior complex scaling to a two-electron system, (c) The spectrum of the Hamiltonian of a multi-electron system, and therefore the analytic structure of its Green's function, in the complex energy plane under complex scaling of either type.

the coordinates of the Hamiltonian,

$$
r \rightarrow \begin{cases}r & \text { for } r \leq R_{0} \\ R_{0}+\left(r-R_{0}\right) e^{i \eta} & \text { for } r>R_{0}\end{cases}
$$

where $R_{0}$ defines the radius within which the wave function will be the usual function of real-valued coordinates, and $\eta$ is a scaling angle. This basic idea in fact appeared in a less formal paper by Nicolaides and Beck in the previous year [39], but Simon showed that the spectrum of the Hamiltonian changes under this transformation in exactly the same way as under the original simple complex scaling transformation in Eq.(11). Thus it was shown that ECS could be used for direct calculations of resonance energies for potentials that are nonanalytic for values of the coordinates less than $R_{0}$.

However, at this point it was not at all apparent that the idea of exterior complex scaling could be used for the calculation of scattering amplitudes for any long-range potential, particularly for the calculation of breakup amplitudes for a 
Coulomb system. To see how ECS has been developed over the past decade into a powerful method for general scattering calculations we must address that problem directly. One component of its solution is the fact that, unlike the simple scaling idea in Eq.(11), ECS transformation in Eq.(12) provides a method for simultaneously imposing outgoing wave boundary conditions while yielding the wave function as a function of real coordinates in the region where the amplitudes, or outgoing flux, can be calculated. Note that in an exact or converged calculation the solutions of the Schrödinger equation for $r<R_{0}$ do not depend on $\eta$, because in that case exterior complex scaling simply produces the exact solution with the coordinates taken on a particular complex contour [38].

As we will discuss below, setting $\eta \neq 0$ while imposing the boundary condition that on the ECS contour the wave function vanish as $r \rightarrow \infty$ effectively imposes outgoing scattering boundary conditions on the exact solution, and that is why the ECS approach provides a path to compute collision amplitudes. It is this property that makes ECS particularly useful in cases where the asymptotic boundary conditions make traditional methods difficult to apply.

To understand the ECS method as applied to atomic systems, we first should recall the basic formulation of ECS from earlier treatments of the subject [40, 41]. In particular we must understand the role of the discontinuity at $r=R_{0}$ of the Jacobian of the ECS transformation in Eq.(12). The easiest way to see the origin of that discontinuity is to first examine the case of arbitrary "smooth" exterior complex scaling where that discontinuity does not appear, which we do briefly in Section 2.2 below, and then specialize to the present case of "sharp" exterior scaling as we do in Section 2.3. This discontinuity is the central question for any basis or grid representation to represent the Schrödinger equation under the ECS transformation. Addressing it correctly is the key to constructing a numerically robust implementation. Once we have dealt with that issue, we will be ready to discuss the issue of the application of ECS to long range potentials in Section 2.4.

\subsection{Formulation of complex scaling for arbitrary complex coordinate contours}

To begin, consider a one-dimensional radial problem for which we make a single-valued and continuous, but otherwise arbitrary, complex transformation of the coordinate $r$ according to $r \rightarrow R(r)$. The operator, $U$, that performs that transformation on the wave function $\psi(r)$ is defined according to

$$
U \psi(r)=J(r) \psi(R(r))
$$

where the Jacobian of the transformation is

$$
J(r)=\left(\frac{d R(r)}{d r}\right)^{1 / 2}
$$

If the original Schrödinger equation is

$$
H \psi(r)=E \psi(r)
$$

with

$$
H=-\frac{1}{2 \mu} \frac{d^{2}}{d r^{2}}+\frac{l(l+1)}{2 \mu r^{2}}+V(r)
$$

then the scaled Schrödinger equation results from a unitary transformation,

$$
U H U^{-1} U \psi=E U \psi
$$


in which the inverse of the scaling operator is given by

$$
U^{-1} \psi=\frac{1}{J\left(R^{-1}(r)\right)} \psi\left(R^{-1}(r)\right)
$$

and $R^{-1}(r)$ is the inverse of the function defining the complex contour.

We can represent the complex contour, $R(r)$, in the general form suggested by Kurasov et al. [41],

$$
R(r)=\int_{0}^{r} q\left(r^{\prime}\right) d r^{\prime}
$$

so that $d R(r)=q(r) d r$ and the exterior scaling transformation is specified by requiring $q(r)$ to satisfy the conditions

$$
q(r) \rightarrow \begin{cases}1 & \text { for } r \rightarrow 0 \\ e^{i \eta} & \text { for } r \rightarrow \infty\end{cases}
$$

In this way we can describe both "smooth" exterior scaling, in which $R(r)$ is continuously differentiable, as well as the "sharp" exterior scaling transformation in Eq.(12). The transformed Schrödinger equation that results is

$$
\hat{H} \phi(r)=E \phi(r)
$$

where $\phi(r)=\psi(R(r))$ and the transformed Hamiltonian has the form

$$
\hat{H}(r)=-\frac{1}{2 \mu}\left[\frac{1}{q(r)^{2}} \frac{d^{2}}{d r^{2}}-\frac{q^{\prime}(r)}{q(r)^{3}} \frac{d}{d r}\right]+\frac{l(l+1)}{2 \mu R(r)^{2}}+V(R(r))
$$

In a basis set expansion the idea is to expand the transformed wave function, $\phi(r)$, and not $U \psi(r)$ appearing in Eq.(13) which contains the Jacobian factor $q(r)^{1 / 2}$, in a set of square-integrable functions $\chi_{n}(r)$. The derivative portion of the kinetic energy matrix elements is therefore defined according to

$$
\begin{aligned}
K_{m n} & =-\frac{1}{2 \mu} \int_{0}^{\infty} \chi_{m}(r)\left[\frac{1}{q(r)^{2}} \frac{d^{2}}{d r^{2}}-\frac{q(r)^{\prime}}{q(r)^{3}} \frac{d}{d r}\right] \chi_{n}(r) q(r) d r \\
K_{m n} & =\frac{1}{2 \mu} \int_{0}^{\infty} \chi_{m}^{\prime}(r) \frac{1}{q(r)} \chi_{n}^{\prime}(r) d r
\end{aligned}
$$

where the second equality, which exhibits the fact that the kinetic energy matrix is complex symmetric, comes from integrating by parts and using the fact that the $\chi_{n}$ vanish at the extrema of the integration range (or grid). The other matrix elements, where the operator is just a function of $R(r)$, are defined in the entirely obvious way, with the potential matrix elements, for example, being given by the integral

$$
V_{m n}=\int_{0}^{\infty} \chi_{m}(r) V(R(r)) \chi_{n}(r) q(r) d r
$$

with the volume element $q(r) d r$. Thus the matrix representation of the Hamiltonian is explicitly complex symmetric for any contour defined by $q(r)$.

Now we are ready to turn to the conditions on $\phi(r)$ and its derivatives at $r=R_{0}$ for the case of "sharp" exterior scaling in Eq.(12) 


\section{3. "Sharp" Exterior scaling and the question of the discontinuity of the Jacobian}

To use the exterior scaling contour originally defined by Eq.(12), we simply set the function $q(r)$ equal to

$$
q(r)= \begin{cases}1 & \text { for } r \leq R_{0} \\ e^{i \eta} & \text { for } r>R_{0}\end{cases}
$$

With this definition the Jacobian

$$
J(r)=q(r)^{1 / 2}
$$

is obviously discontinuous because $q(r)$ is discontinuous. The transformed wave function $\phi(r)=\psi(R(r))$ is always continuous, although its first derivative with respect to $r$ is not continuous because

$$
\frac{d}{d r} \phi(r)=\frac{d R(r)}{d r} \psi^{\prime}(R(r))=q(r) \psi^{\prime}(R(r))
$$

Note that the underlying wave function $\psi(z)$ is an analytic function of its argument, $z$, and its derivative with respect to that argument, $\psi^{\prime}(z)$, is continuous in any direction in the complex plane as demanded by the Cauchy conditions on the derivatives of analytic functions of a complex variable. The only discontinuity in Eq.(27) is that of $q(r)$. With sharp exterior scaling, we always use Eq. $(23 b)$ to define the kinetic energy.

In every implementation of ECS using a basis set expansion it is exactly this point that is critical, because the numerical convergence properties depend on the correct representation of the wave function at $r=R_{0}$. Fortunately, as the list of successful applications of ECS attests, it is generally not difficult to treat the the discontinuity at the point $R_{0}$ exactly. For example, in the implementation in terms of the discrete variable representation and finite elements [42], we will point out below that putting a DVR finite element boundary point at $R_{0}$ and requiring only continuity of the value of the function at that point gives the DVR basis the ability to represent $\phi(r)$ with continuous value at $R_{0}$, but with discontinuous derivative. The result of such a calculation is illustrated in Fig. 2. Other impementations describe the details differently, but accomplish the same end [43].

In general, basis functions of compact support can treat the condition at $R_{0}$ exactly, while complex analytic basis set expansions [40, 44], like expansions in Slater or Gaussian functions cannot. The successful use of complex analytic basis set expansions, for example Slater or Gaussian basis functions, with ECS generally requires "smooth" exterior scaling in which $q(r)$ is continuous and continuously differentiable [45, 46]. Methods based on grids or on the use of basis functions of compact support, however, can treat the "sharp" exterior scaling of Eq.(12) exactly.

\subsection{Making complex scaling work for long range potentials}

To understand the question of how the range of the potential affects the application of complex scaling of the coordinates in the Schrödinger equation, we can continue with the single particle example of the previous sections. First we express the scattering wave function at the energy, $E=k^{2} / 2 \mu$, in a form that we will use in every application of complex scaling to the breakup problem we will discuss in this review,

$$
\psi^{+}=\psi^{s c}+\psi_{0}
$$

where $\psi_{0}$ defines the incoming boundary conditions of the scattering problem. In a one particle example we can take $\psi_{0}(r)=j_{l}(k r)$, where $j_{l}(k r)$ is the Ricatti-Bessel function 
[47]. Then we get the driven Schrödinger equation that is the typical starting point for exterior complex scaling calculations, which we can specialize to a one-particle example,

$$
\begin{aligned}
& (E-H) \psi^{s c}=(H-E) \psi_{0} \\
& (E-H) \psi^{s c}(r)=V(r) j_{l}(k r)
\end{aligned}
$$

In Eq.(29b) the Hamiltonian is that in Eq.(16). We now seek the solution for $\psi^{s c}$ satisfying outgoing boundary conditions. If the the potential, $V(r)$, diminishes at large $r$ faster than $1 / r^{2}$,

$$
\psi^{s c}(r) \underset{r \rightarrow \infty}{\longrightarrow} A h_{l}^{+}(k r)
$$

where $A$ is the scattering amplitude and $h_{l}^{+}(z)$ is the outgoing Ricatti-Hankel function [47].

Now, if we try to solve Eq. $(29 b)$ on the original rotated coordinates contour in Eq.(11), the right hand side becomes $V\left(r e^{i \eta}\right) j_{l}\left(k r e^{i \eta}\right)$. Because the Bessel function has the asymptotic form $\sin \left(k r e^{i \eta}-l \pi / 2\right)$, it diverges exponentially as a function of $r$ on the contour. The right hand side thus diverges, unless the potential is of finite range or is exponentially bounded with an exponent sufficiently large to overcome the exponentially diverging Bessel function on the contour. Although the problem is expressed differently by Nuttall and Cohen [37], who instead focus on the formal expression for the T-matrix,

$$
T=\lim _{\epsilon \rightarrow 0}\left\langle\psi_{0}\left|V+V(E-H+i \epsilon)^{-1} V\right| \psi_{0}\right\rangle
$$

the argument is exactly the same, and it limits the utility of ordinary complex scaling in this context.
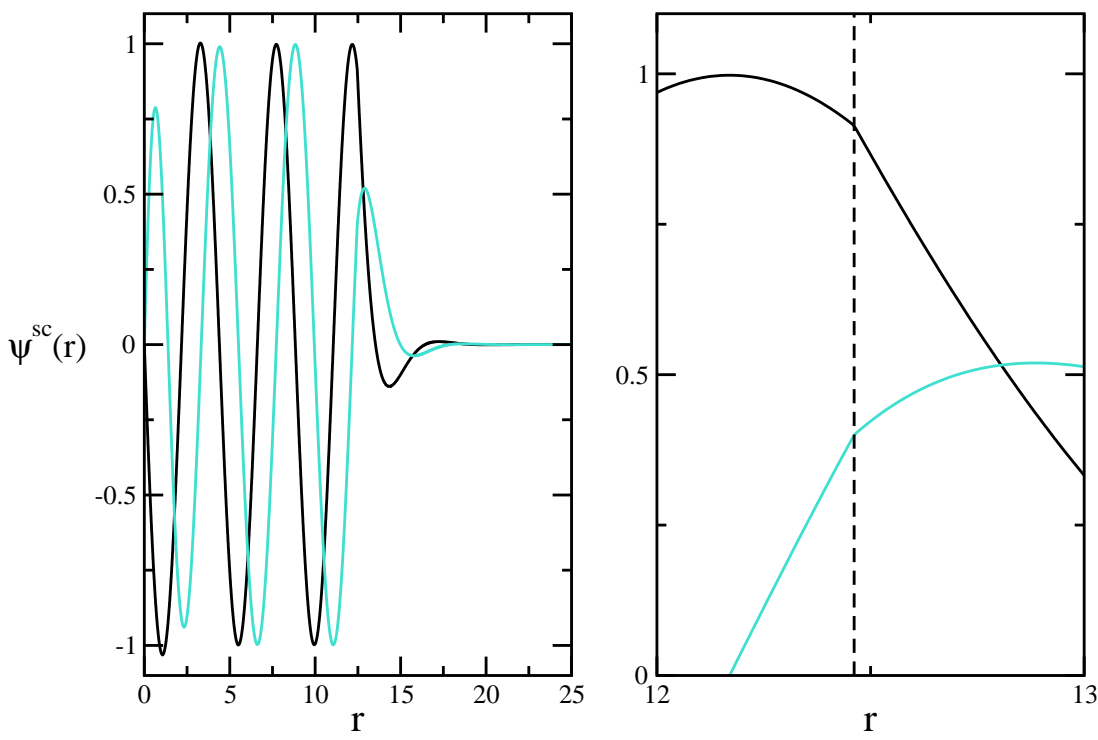

Figure 2. Scattered wave function for the potential $V=-3 e^{-r}$ under exterior complex scaling with $\eta=40^{0}$ showing $\operatorname{Re}\left[\Psi^{s c}\right]$ (solid black) and $\operatorname{Im}\left[\Psi^{s c}\right]$ (solid grey). Right panel shows derivative discontinuity at $r=R_{0}$ (dashed line). 
However, Rescigno et al. [40] showed how exterior complex scaling can overcome this difficulty. Consider Eq.(29b) on the exterior complex scaling contour in Eq.(12). Once again the right hand side evaluated on the contour, $V(R(r)) j_{l}(k R(r))$ diverges for large $\mathrm{r}$, but it begins to do so only for $r>R_{0}$. That is a key point, because it allows the following procedure. If we replace the original potential (on the right hand side) by the finite range potential, $V_{R_{0}}(r)$, defined by truncating $V(R)$ at $R_{0}$,

$$
V_{R_{0}}(r)= \begin{cases}V(r) & \text { for } r \leq R_{0} \\ 0 & \text { for } r>R_{0}\end{cases}
$$

we can use Eq.(29b) with exterior scaling to solve for $\psi_{R_{0}}^{s c}(r)$ from

$$
(E-H) \psi_{R_{0}}^{s c}(r)=V_{R_{0}}(r) \psi_{0}(r)
$$

because right hand side no longer diverges. $\psi_{R_{0}}^{s c}(r)$ still satisfies pure outgoing wave boundary conditions, and exterior complex scaling converts the pure outgoing wave boundary condition to

$$
\psi_{R_{0}}^{s c}(R(r)) \underset{r \rightarrow \infty}{\longrightarrow} 0 .
$$

This equation is true because the Ricatti-Hankel function, like any outgoing wave solution of the one- or many-particle Schrödinger equation, goes to zero in the upper half $r$-plane if it is associated with a real-valued momentum.

Thus we can solve for $\psi_{R_{0}}^{s c}(r)$ by representing Eq.(33) on a finite basis or grid, applying the boundary conditions that the solution vanish at $r=0$ and $r \rightarrow \infty$ (or at $r=r_{\max }$, the maximum value of $r$ on the grid), and solving the resulting linear equations. In Section 3 we will discuss in detail how to implement such a calculation.

The key point here is that such a calculation yields $\psi_{R_{0}}^{s c}(r)$ as a function of real $r$ for $r<R_{0}$. In a practical calculation we will make use of that wave function for $r<R_{0}$ to extract the scattering amplitudes from it. Thus we have the important property of the solution of the ECS version of Eq.(33) that

$$
\psi_{R_{0}}^{s c}(R(r)) \underset{R_{0} \rightarrow \infty}{\longrightarrow} \psi^{s c}(R(r))
$$

for $r<R_{0}$ and thus ultimately for all $r$.

In a more formal way, we can express this result as follows. Exterior complex scaling of the driven Schrödinger equation, Eq.(29b) and its many-particle generalization, produces the correct physical solution for $\psi^{s c}$, if we first take the $r \rightarrow \infty$ limit, use ECS to apply the outgoing wave boundary conditions, and then take the $R_{0} \rightarrow \infty$ limit. The order of the limits matters, because it allows the avoidance of the problem that originally limited the application of ordinary complex scaling to the calculation of scattering amplitudes for long-range potentials.

In a practical calculation, the value of $R_{0}$ can be increased until the calculation of scattering amplitudes and cross sections converges to arbitrary accuracy. That value of course depends on the energy, with lower values of the energy requiring large values of $R_{0}$, just as calculations by any method near threshold require the representation of the Schrödinger equation on a larger spatial region. In any case, the scattering amplitude can easily be extracted in the region $r<R_{0}$ from its definition in Eq.(30). The methods for doing that for a multi-electron problem will be discussed below, but for our simple example the scattering amplitude is clearly just,

$$
A=\frac{2 \mu}{k}\left\langle j_{l}(k r)|V(r)| \Psi^{+}(r)\right\rangle
$$




$$
\begin{aligned}
& =\frac{2 \mu}{k}\left\langle j_{l}(k r)|T-E| \psi^{s c}(r)\right\rangle_{R_{0}} \\
& =-\left.\frac{1}{k} W\left(j_{l}(k r), \psi^{s c}(r)\right)\right|_{r=R_{0}-\epsilon}
\end{aligned}
$$

where in Eq.(36b) the subscript $R_{0}$ denotes integration over the "volume", $r \leq R_{0}$, and in Eq. (36c) $W(a, b)=a^{\prime} b-b^{\prime} a$ denotes the Wronskian with respect to $r$. One should also note that there is a "two-potential" version of this formalism [48] that would, for example, allow the use of a Coulomb potential as a reference potential in electron-ion scattering.

We now turn in Section 3 to the practical issues of representing Eq.(29a) in one or more dimensions on the exterior complex scaling contour $R(r)$. Note that it is to "sharp" exterior complex scaling, Eq.(12), that the arguments above about the order of the limits most directly apply, and, as we will see, "sharp" ECS has entirely benign numerical properties as well.

\section{Numerical implementations of exterior complex scaling}

Although it might seem at first glance that "smooth" exterior complex scaling should be numerically better behaved than "sharp" ECS, in fact the opposite is true. The issue revolves around the question of the representation of the discontinuity in the derivative of the wave function for sharp ECS, illustrated in Fig. 2, versus the representation of the smoothed version of that discontinuity in smooth ECS.

It has been our experience [40] that smooth scaling can give rise to errors that can depend sensitively on the way in which the contours are smoothed. The point of "smooth" scaling is to make it possible to implement the method with analytic basis sets $[44,45,46]$ such as Gaussian or Laguerre basis functions, although "smooth" ECS inevitably complicates the evaluation of the matrix elements. More importantly such implementations rely on the analytic basis functions to represent the rapidly changing derivative where the ECS contour "turns the corner" into the complex plane. For that reason additional numerical error is introduced by this approach that we have found difficult to eliminate.

On the other hand, if we choose to implement ECS by using either a numerical grid method or with basis functions that have compact support, then the derivative discontinuity at $R_{0}$ that occurs with "sharp" exterior scaling can be handled exactly, provided one correctly chooses $R_{0}$ to coincide with a grid point. Numerical errors are generally much better controlled with such approaches [40]. It is for this reason that we have chosen in all our implementations of ECS to use grid-based methods and "sharp" exterior scaling.

The implementations we will discuss in this section are ECS approaches that have been used for the Coulomb breakup problem: 1) finite differences, 2) the discrete variable representation combined with finite elements, 3) B-splines, and 4) direct

numerical integration. For multiphoton ionization in the context of Floquet theory, Chu and coworkers [43] have very successfully made use of "sharp" exterior scaling in yet another implementation.

The task of the numerical implementations of ECS is to make a finite representation of Eq. $(29 a)$, and generally that representation will lead to linear equations for the the coefficients that define $\psi^{s c}$. In methods involving a basis set expansion (with compact support in these examples) we require matrix elements of the kinetic energy, of the potential energy (or any local function of the coordinates), 
and of the overlap if the basis is nonorthogonal. These matrix elements are given by the appropriate versions of Eq.(23b) and Eq.(24) with $q(r)$ defined as in Eq.(25). Most often the matrix representation of the Hamiltonian will be complex symmetric as it is in the discrete variable representation and B-spline methods described below, although as we will see in the case of finite differences, that need not be the case.

\subsection{Finite differences}

The most obvious way to implement exterior complex scaling is with finite difference approximations. To do so we choose a grid of points in $r$ with one of the grid points $r_{i}$ chosen to be equal to $R_{0}$. The complete complex finite difference grid is then the set of points, $R\left(r_{i}\right)$, and thus lies on the contour. On the entire ECS contour the potential can be simply represented by its values, $V\left(R\left(r_{i}\right)\right)$. The kinetic energy requires considerably more care, however.

Normally we speak of a "stencil" with $2 p+1$ points in $r\left(r_{-p}, r_{-p+1}, \cdots, r_{p}\right)$ for the finite difference approximation to the second derivative. The familiar three-point formula for the second derivative would correspond to a stencil with $p=1$. At most points on the real and complex portions of the grid, ordinary finite difference formulas for the derivative will apply. However, consider the points where the stencil straddles the point $R_{0}$ where the complex part of the ECS contour begins. In that case, if we are taking points equidistant along the contour, points to the right of $R_{0}$ are given by $R_{0}+j \Delta e^{i \eta}$ while those to the left of $R_{0}$ are given by $R_{0}-j \Delta$, where $j$ is an integer. For these cases we must make use of formulas for unequally spaced points, because the cancellations that yield the usual finite difference formulas for derivatives require that the steps be taken with the same magnitude and phase.

To derive general formulas for those few cases where the stencil overlaps the point $R_{0}$, we can proceed by first writing the standard approximation to the function in terms of Lagrange interpolating polynomials [49].

$$
\psi(r)=\sum_{i=-p}^{p} l_{i}(r) \psi\left(r_{i}\right)+R_{2 p}(r)
$$

where the Lagrange interpolating polynomial is defined by

$$
\begin{aligned}
l_{i}(r) & =\frac{\pi_{2 p}(r)}{\left(r-r_{i}\right) \pi_{2 p}^{\prime}\left(r_{i}\right)} \\
& =\frac{\left(r-r_{-p}\right) \cdots\left(r-r_{i-1}\right)\left(r-r_{i+1}\right) \cdots\left(r-r_{p}\right)}{\left(r_{i}-r_{-p}\right) \cdots\left(r_{i}-r_{i-1}\right)\left(r_{i}-r_{i+1}\right) \cdots\left(r_{i}-r_{p}\right)}
\end{aligned}
$$

with

$$
\pi_{2 p}(r)=\prod_{i=-p}^{p}\left(r-r_{i}\right)
$$

and where the remainder term, $R_{2 p}(r)$ is given by

$$
R_{2 p}(r)=\pi_{2 p}(r) \frac{\psi^{(p+1)}(\zeta)}{(2 p+1) !} \quad r_{-p}<\zeta<r_{p}
$$

Now to derive any finite difference approximation to the second derivative that we might require, we merely differentiate the formula in Eq.(37) and evaluate the result 
at the central point, $r_{0}$ (for higher orders, a task most easily and safely accomplished with one of the standard computer algebra packages).

$$
\psi_{0}^{\prime \prime}=\psi^{\prime \prime}\left(r_{0}\right)=\left.\frac{d^{2}}{d r^{2}} \psi(r)\right|_{r=r_{0}}
$$

The derivative of the remainder term gives the error estimate.

Applying this procedure to the simplest case, $p=1$ we get the three-point finite difference formulas that apply to this case,

$$
\begin{aligned}
\psi_{j}^{\prime \prime} & =\frac{1}{\Delta^{2}}\left(\psi_{j-1}-2 \psi_{j}+\psi_{j+1}\right)+\mathrm{O}\left(\Delta^{2}\right) \\
\psi_{n}^{\prime \prime} & =\frac{1}{\Delta^{2}}\left(\frac{2}{\left(1+e^{i \eta}\right)} \psi_{n-1}-2 e^{-i \eta} \psi_{n}+\frac{2 e^{-i \eta}}{\left(1+e^{i \eta}\right)} \psi_{n+1}\right)+\mathrm{O}(\Delta) \\
\psi_{m}^{\prime \prime} & =\frac{e^{-2 i \eta}}{\Delta^{2}}\left(\psi_{m-1}-2 \psi_{m}+\psi_{m+1}\right)+\mathrm{O}\left(\Delta^{2}\right)
\end{aligned}
$$

where Eq.(42a) applies on the real part, Eq.(42b) (incidentally, not symmetric with respect to the coefficients of $\psi_{n-1}$ and $\psi_{n+1}$ ) applies at the point $r_{n}=R_{0}$, and Eq. $(42 c)$ applies on the complex part of the ECS contour. One immediately sees a critical drawback of the finite difference approach. While the error is second order at all other points, the error in the second derivative at the critical point $r_{n}=R_{0}$ is first order in the stepsize, $\Delta$. In general, the error in an $n$-point formula will be one order lower for those points where the stencil overlaps the point $R_{0}$ than elsewhere on the grid.

For that reason higher orders are essential in practical calculations, and a sevenpoint stencil was used when the first large-scale implementation of the finite-difference approach for ECS was applied to electron-impact ionization of the hydrogen atom [50, 51], and Baertschy et al. [52] give explicit formulas, and error estimates, for the necessary derivative formulas. It should be noted that even when using three-point finite difference for the second derivative, the discontinuity in the first derivative in Eq.(27) is represented exactly in a numerical calculation by the corresponding twopoint formula for the first derivative.

The finite difference method has an advantage over most basis set, or "spectral", methods in that it involves a trivially simple diagonal representation of the potential. However since higher order finite difference is necessary for accurate calculations, spectral methods which lead to more compact representations of the wave function and therefore smaller sets of equations, are generally preferable for implementing ECS. A spectral method that preserves the advantage of a diagonal representation of the potential, while producing a much more accurate representation of the kinetic energy is the finite-element discrete variable representation (FEM-DVR) approach to which we turn next.

\subsection{Finite Elements and the Discrete Variable Representation}

The discrete variable representation (DVR) [53] combines a high order treatment of the kinetic energy operator in a polynomial basis with the advantage of a diagonal representation of the potential operator, i.e., one that does not require the computation of matrix elements of the potential. The DVR was combined with the finite elements method (FEM) to allow the treatment of exterior complex scaling by Rescigno and McCurdy [42], and this approach arguably provides the most efficient implementation of ECS for multielectron systems to date. In a multielectron problem the electronic 
repulsion potential, $1 /\left|\mathbf{r}_{i}-\mathbf{r}_{j}\right|$, presents a computational challenge to any numerical method, but as we will show below, the finite-element method with discrete variable representation (FEM-DVR) approach provides a particularly efficient representation that is effectively diagonal. The FEM-DVR approach is therefore far superior to the earlier efforts to make use of the finite-element method for ECS based on nonorthogonal polynomial basis functions $[54,55]$.

The DVR that we employ takes the analysis of Manolopoulos and Wyatt [56] as its point of departure and uses Langrange interpolating polynomials with mesh points derived from a Gauss-Lobatto quadrature. Gauss-Lobatto quadrature is similar to the more familiar Gauss-Legendre quadrature, both of which approximate integrals as,

$$
\int_{a}^{b} F(x) d x \approx \sum_{i=1}^{n} F\left(x_{i}\right) w_{i}
$$

In Gauss-Lobatto quadrature, two of the points are constrained to coincide with the end points, and that means that Eq.(43) can be made exact when $F(x)$ is a polynomial of degree $\leq 2 n-1$. On the interval $[a, b]$ one can define normalized DVR basis functions as

$$
f_{i}(x)=w_{i}^{-1 / 2} \prod_{j \neq i} \frac{x-x_{j}}{x_{i}-x_{j}}
$$

These functions have the property, when evaluated at the quadrature points, that

$$
f_{i}\left(x_{j}\right)=\delta_{i, j} / \sqrt{w_{i}}
$$

and they are thus orthonormal under Gauss-Lobatto integration,

$$
\int_{a}^{b} f_{i}(x) f_{j}(x) d x \approx \sum_{k=1}^{n} f_{i}\left(x_{k}\right) f_{j}\left(x_{k}\right) w_{k}=\delta_{i, j}
$$

Since Gauss-Lobatto quadrature explictly includes the end points as quadrature points, it is possible to combine this particular varitey of DVR with the finiteelement method. In the FEM-DVR approach we divide the ECS contour for the radial coordinate of each electron into one-dimensional finite elements with one of the boundaries coinciding with the point $R_{0}$ where the real and complex parts of the contour join. In each element we use the same order, $n$, of Gauss-Lobatto quadrature. For each independent variable, $r$, (radial coordinate of an electron) we choose a grid of $N$ nodes, $r^{(j)}$, that consists of the union of all the Gauss-Lobatto mesh points of the separate finite elements, and we arrange them in ascending order, $0 \leq r^{(1)}<r^{(2)}<\cdots<r^{(N)}$.

We then define a set of DVR functions in each interval, in a notation where $f_{i}^{m}(r)$ refers to the $i$ th DVR function in the $m$ th element. We can impose continuity conditions across elements by combining the end-point functions on adjacent intervals into single "bridging" functions, $\chi_{i}(r)$,

$$
\chi_{i}(r)=\left[f_{n}^{i}(r)+f_{1}^{i+1}(r)\right] / \sqrt{w_{n}^{i}+w_{1}^{i+1}}
$$

Thus we can impose the boundary conditions that the wave function vanish at the grid boundaries by excluding the basis functions centered on the first and last nodes.

On the real part of the ECS countour, Eq.(44) and Eq.(47) apply as written. On the complex part of the contour these equations are modified to reflect integration 
along the contour, so that beyond the point $r_{0}$ we have

$$
\begin{aligned}
& f_{j}^{i}\left(r_{k}^{i}\right)=\delta_{j, k} / \sqrt{w_{j} e^{i \eta}} \\
& \chi_{i}(r)=\left[f_{n}^{i}(r)+f_{1}^{i+1}(r)\right] / \sqrt{\left(w_{n}^{i}+w_{1}^{i+1}\right) e^{i \eta}}
\end{aligned}
$$

One basis function remains to be defined, the bridging function associated with the finite element boundary at $r_{n}^{m}=r_{1}^{m+1}=R_{0}$, and that function is

$$
\chi_{m}(r)=\left[f_{n}^{m}(r)+f_{1}^{m+1}(r)\right] / \sqrt{w_{n}^{m}+w_{1}^{m+1} e^{i \eta}}
$$

We can now arrange all the FEM-DVR functions, including the bridging functions, into a single basis $\left\{\phi_{1}(r), \cdots, \phi_{N}(r)\right\}$, where each function is associated with a mesh point, $r^{(i)}$. The FEM-DVR basis is illustrated in Fig. 3, where it is plotted over two finite elements for a low order DVR. In this basis, the matrix elements of any one-body potential are diagonal, regardless of which grid points, real or complex, are involved.

$$
\int_{0}^{\infty} \phi_{i}(r) V(r) \phi_{j}(r) d r=\delta_{i, j} V\left(r^{(i)}\right)
$$

The kinetic energy is not diagonal, however, and in the basis of the functions $\left\{\phi_{i}(r)\right\}$ has a blocked structure that can be deduced from the definitions of the FEM-DVR basis functions [42]

Multi-electron problems present another difficulty because of the singularity of the electronic repulsion potential, $1 /\left|\mathbf{r}_{i}-\mathbf{r}_{j}\right|$. For atomic problems, or in any case where we make use of a single-center expansion basis, we define the three-dimensional basis functions as products of our FEM-DVR radial functions and spherical harmonics,

$$
\Phi_{i \ell m}(\mathbf{r})=\phi_{i}(r) Y_{\ell m}(\theta, \varphi)
$$

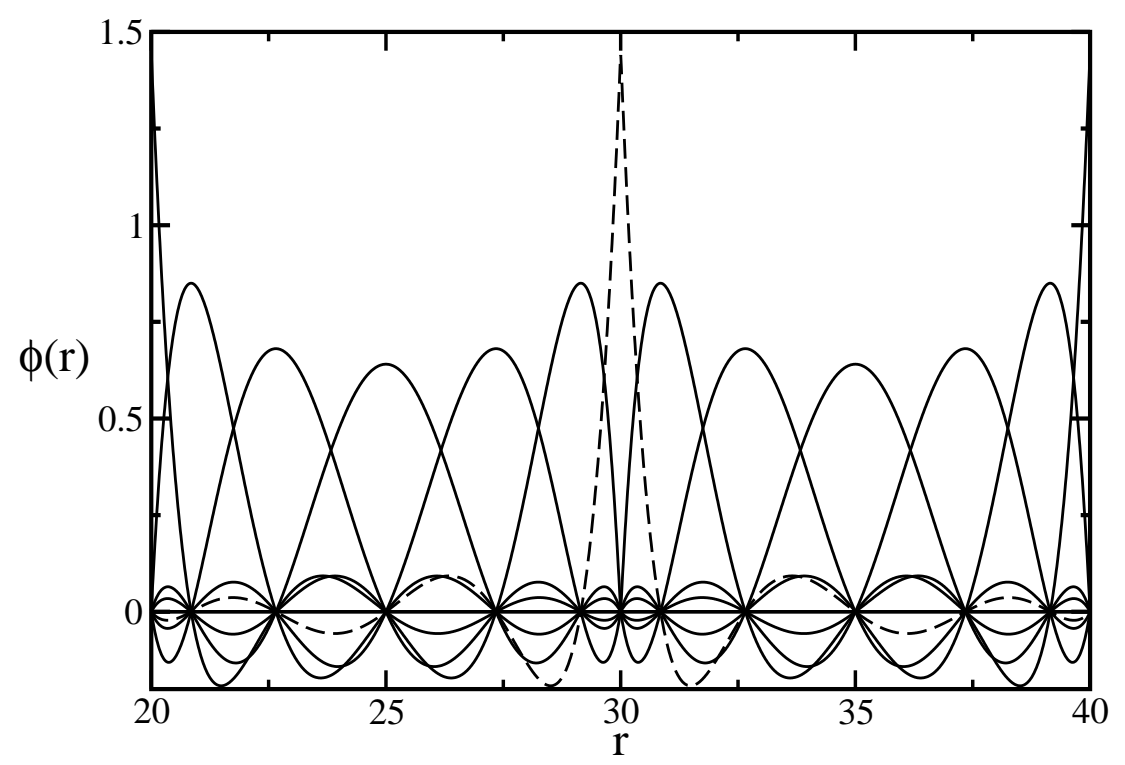

Figure 3. FEM-DVR basis plotted over two finite elements for 7 th order GaussLobatto quadrature (solid curves). The central bridging function is plotted as a dashed curve. 
The two-electron integrals we must compute are thus of the form

$$
\left\langle\Phi_{i \ell_{1} m_{1}}\left(\mathbf{r}_{1}\right) \Phi_{j \ell_{2} m_{2}}\left(\mathbf{r}_{2}\right)\left|\frac{1}{\left|\mathbf{r}_{1}-\mathbf{r}_{2}\right|}\right| \Phi_{k \ell_{3} m_{3}}\left(\mathbf{r}_{1}\right) \Phi_{l \ell_{4} m_{4}}\left(\mathbf{r}_{2}\right)\right\rangle
$$

where the integrations are over both electronic coordinates. and are evaluated by making a multipole expansion of the interelectron repulsion

$$
\frac{1}{\left|\mathbf{r}_{1}-\mathbf{r}_{2}\right|}=\sum_{\ell} \frac{r_{<}^{\ell}}{r_{>}^{\ell+1}} P_{\ell}\left(\cos \theta_{12}\right)
$$

The angular parts of each integral can be done analytically, reducing the problem to the evaluation of radial integrals. The generic radial integral that results in each case has the form

$$
\left\langle\rho_{B}\left|\frac{r_{<}^{\ell}}{r_{>}^{\ell+1}}\right| \rho_{A}\right\rangle=\int_{0}^{r_{\max }} d r \int_{0}^{r_{\max }} d r^{\prime} \rho_{B}(r) \frac{r_{<}^{\ell}}{r_{>}^{\ell+1}} \rho_{A}\left(r^{\prime}\right)
$$

where the densities $\rho_{A}(r)$ and $\rho_{B}(r)$ are products of our radial FEM-DVR basis functions

$$
\begin{aligned}
& \rho_{A}(r)=\phi_{i}(r) \phi_{k}(r) \\
& \rho_{B}(r)=\phi_{j}(r) \phi_{l}(r)
\end{aligned}
$$

and $r_{\max }$ denotes the end of the ECS contour.

Gauss-Lobatto quadrature is not valid for the integrations in Eq.(55), because because it effectively expands the derivative discontinuity in the potential $r_{<}^{\ell} / r_{>}^{\ell+1}$ in a basis of polynomials. Therefore replacing this potential with its values on at the mesh points is a poor approximation to the integral. However we can restore the validity of the underlying Guass quadrature if instead we apply the DVR to solving Poisson's equation for the potential due to the charge distribution corresponding to a product of two of the FEM-DVR basis functions.

First we define the function $y(r)$

$$
\begin{aligned}
& y(r)=r \int_{0}^{r_{\max }} \rho_{A}\left(r^{\prime}\right) \frac{r_{<}^{\ell}}{r_{>}^{\ell+1}} d r^{\prime} \\
& =\int_{0}^{r} \rho_{A}(t)\left(\frac{t}{r}\right)^{\ell} d t+\int_{r}^{r_{\max }} \rho_{A}(t)\left(\frac{r}{t}\right)^{\ell+1} d t
\end{aligned}
$$

so that the original integral can be written as a simple quadrature

$$
\left\langle\rho_{B}\left|\frac{r_{<}^{\ell}}{r_{>}^{\ell+1}}\right| \rho_{A}\right\rangle=\int_{0}^{r_{\max }} \rho_{B}(r) \frac{1}{r} y(r) d r
$$

Differentiation of $y(r)$ with respect to $r$ shows that it satisfies the radial form of Poisson's equation

$$
\left(\frac{d^{2}}{d r^{2}}-\frac{l(l+1)}{r^{2}}\right) y(r)=-\frac{2 l+1}{r} \rho_{A}(r)
$$

with the boundary conditions

$$
\begin{aligned}
& y(0)=0 \\
& y\left(r_{\max }\right)=\frac{1}{r_{\max }^{\ell}} \int_{0}^{r_{\max }} \rho_{A}(t) t^{\ell} d t=\frac{1}{r_{\max }^{\ell}} \delta_{i, k} r_{i}^{\ell}
\end{aligned}
$$

where in the last equality we performed the integral with Gauss-Lobatto quadrature and used the properties of the DVR basis functions. 
The central idea now is to solve this equation by expanding $y(r)$ in the same FEM-DVR basis with which we are solving the Schrödinger equation. However, our FEM-DVR basis satisfies only the first of these boundary conditions. It is useful to recall [57] that the radial Green's function for Poisson's equation satisfies

$$
\left(\frac{d^{2}}{d r^{2}}-\frac{\ell(\ell+1)}{r^{2}}\right) r g\left(r, r^{\prime}\right)=-\frac{2 \ell+1}{r} \delta\left(r-r^{\prime}\right)
$$

and that on the interval $(0, b)$ that Green's function (for two-point boundary conditions) is

$$
g\left(r, r^{\prime}\right)=\frac{r_{<}^{\ell}}{r_{>}^{\ell+1}}-\frac{r^{\ell} r^{\ell}}{b^{2 \ell+1}}
$$

The second term in this Green's function suggests the method we will use to solve Eq.(59).

The first step is to seek a solution for $y(r)$ that satisfies the boundary condition $y(0)=0$, but that in place of the correct boundary condition at $r_{\max }$ satisfies $y\left(r_{\max }\right)=0$. We expand that solution in the basis of FEM-DVR functions, which satisfy the boundary conditions that they vanish at 0 and $r_{\text {max }}$,

$$
y^{(0)}(r)=\sum_{m=1}^{N} C_{m} \phi_{m}(r)
$$

Substituting this expansion in Eq.(59), multiplying by one of the FEM-DVR basis functions from the left and integrating over $r$ gives a matrix equation for the coefficients $C_{m}$.

$$
\sum_{m=0}^{N} T_{n, m}^{(\ell)} C_{m}=(2 \ell+1) \int_{0}^{r_{\max }} \phi_{n}(r) \frac{1}{r} \rho_{A}(r) d r=(2 \ell+1) \frac{\delta_{n, i} \delta_{i, k}}{r_{n} \sqrt{w_{n}}}(
$$

where the matrix, $T_{n, m}^{(\ell)}$ is defined by

$$
T_{n, m}^{(\ell)}=-\int_{0}^{r_{\max }} \phi_{n}(r)\left(\frac{d^{2}}{d r^{2}}-\frac{\ell(\ell+1)}{r^{2}}\right) \phi_{m}(r) d r
$$

Equation(65) has the solution

$$
C_{i}=(2 \ell+1) \frac{\left[T_{m, i}^{(\ell)}\right]^{-1} \delta_{i, k}}{r_{i} \sqrt{w_{i}}}
$$

with $\left[T_{m, i}^{(\ell)}\right]^{-1}$ denoting the $m, i$ element of the matrix inverse, and so gives us the function $y^{(0)}(r)$.

To obtain a solution of Poisson's equation that has the proper boundary conditions we add a term which is a solution of the homogeneous equation corresponding to Eq. (59). The term we add is an exact homogeneous solution, and is analogous to the second term in the Green's function in Eqs.(62) and (63).

$$
\begin{aligned}
& y(r)=y^{(0)}(r)+\frac{r^{\ell+1}}{r_{m a x}^{2 \ell+1}} \int_{0}^{r_{\max }} \rho_{A}\left(r^{\prime}\right) r^{\prime \ell} d r^{\prime} \\
& =(2 \ell+1) \sum_{i=1}^{N} \phi_{m}(r)\left[T_{m, i}^{(\ell)}\right]^{-1} \frac{\delta_{i, k}}{r_{i} \sqrt{w_{i}}}+\frac{r^{\ell+1}}{r_{m a x}^{2 \ell+1}} \delta_{i, k} r_{i}^{\ell}
\end{aligned}
$$


Now that we have a solution for $y(r)$ satisfying the correct boundary conditions in Eq.(61), we can substitute it back into the original expression for the two-electron integral, Eq.(58), to obtain

$$
\begin{aligned}
\left\langle\rho_{B}\left|\frac{r_{<}^{\ell}}{r_{>}^{\ell+1}}\right| \rho_{A}\right\rangle & =\left\langle\phi_{j} \phi_{l}\left|\frac{r_{<}^{\ell}}{r_{>}^{\ell+1}}\right| \phi_{i} \phi_{k}\right\rangle \\
& =\delta_{j, l} \delta_{i, k}\left(\frac{(2 \ell+1)}{r_{j} \sqrt{w_{j}} r_{i} \sqrt{w_{i}}}\left[T_{j, i}^{(\ell)}\right]^{-1}+\frac{r_{j}^{\ell} r_{i}^{\ell}}{r_{m a x}^{2 \ell+1}}\right)
\end{aligned}
$$

The expression in Eq.(70) is the final result for the two-electron integrals in the FEM-DVR basis. It has the remarkable property of being diagonal in the indices corresponding to the FEM-DVR mesh points on the exterior complex scaling contour.

It involves only the inverses, $\left[T_{j, i}^{(\ell)}\right]^{-1}$, of the kinetic energy matrices (times 2 ) in one dimension corresponding to each $\ell$ value, which need only be calculated once, no matter how many two-electron integrals need to be computed. Moreover it maintains the accuracy of the original Gauss-Lobatto quadrature upon which the FEM-DVR approach is based.

\subsection{B-splines}

B-splines have been applied to the ECS transformation by McCurdy and Martín [48] and used in double photoionization calculations by McCurdy et al. [58]. To begin we need the definition of the B-splines themselves as given for example by de Boor [59]. We specify a series of knots $t_{i} \leq t_{i+1}$ in the coordinate, $r$, and the splines of order $k$ are defined by a recursion relation,

$$
B_{i}^{k}(r)=\frac{r-t_{i}}{t_{i+k-1}-t_{i}} B_{i}^{k-1}(r)+\frac{t_{i+k}-r}{t_{i+k}-t_{i+1}} B_{i+1}^{k-1}(r)
$$

together with the definition of B-splines of order $k=1$

$$
B_{i}^{1}(r)= \begin{cases}1 & \text { for } t_{i} \leq r<t_{i+1} \\ 0 & \text { otherwise }\end{cases}
$$

Once the recursion is taken to third order $(k=3)$ one has a set of the familiar (smooth) quadratic splines. Higher orders provide more spline functions in the basis covering successively larger numbers of knots as the order is increased. They also give more continuous derivatives as the order is increased.

The basis of B-splines is entirely defined by the knots $\left\{t_{i}\right\}$. These are built from a grid in the $r$ coordinate (the breakpoint sequence $\xi_{i}$ ) by assigning a multiplicity to each grid point (breakpoint). Thus the positions of the breakpoints coincide with those of the knotpoints, but several knotpoints may coincide with a single breakpoint when multiplicity is larger than one. In most applications, multiple breakpoints are only used near the borders (e.g. at the origin to provide more flexibility in this region). Thus the number of knotpoints is only slightly larger than the number of breakpoints. Details of this technology have been reviewed at length elsewhere [60] and will not be further described here.

What concerns us here is the question of how B-splines can be used to represent the discontinuity of derivative at $r=R_{0}$, that is required for ECS. B-splines of order $k$ have continuous derivatives up to order $k-2$. To represent a function that has a discontinuous derivative of the form of Eq.(27) we define the splines as $B_{i}^{k}(R(r))$. That 
is, put the breakpoints, $\xi_{i}$ and therefore the knots, $t_{j}$ on the complex exterior scaling contour, and place one of the breakpoints and its corresponding knot at $t_{j}=R_{0}$. Then $B_{i}^{k}(R(r))$ has a discontinuous first derivative with respect to $r$ at $r=R_{0}$, because the derivative of $R(r)$ is discontinuous at that point. Furthermore the discontinuity in the first derivative of all the B-splines that span the point $R_{0}$ can be shown to be exactly that of Eq.(27).

Fig. 4 shows the resulting B-splines, at order $k=8$, and demonstrates clearly that the derivatives of the B-splines are discontinuous. In the figure one sees that either the real or the imaginary part of each B-spline shows a discontinuous slope. Another interesting property of ECS B-splines is that the B-splines that do not straddle the point $R_{0}$ are real, whether they are on the complex part of the contour or not.

With the breakpoints defined on the exterior scaling contour, and with one of them conciding with $R_{0}$, the matrix elements of one-body operators are simply sums of the corresponding integrals between breakpoints. For the overlap matrix elements or matrix elements of the potential $V(r)$ we have simply

$$
\begin{aligned}
S_{m n} & =\sum_{l} \int_{\xi_{l}}^{\xi_{l+1}} B_{n}^{k}(r) B_{m}^{k}(r) d r \\
V_{m n} & =\sum_{l} \int_{\xi_{l}}^{\xi_{l+1}} B_{n}^{k}(r) V(r) B_{m}^{k}(r) d r
\end{aligned}
$$

The kinetic energy (times 2) is given by

$$
T_{m, n}=-\int_{0}^{r_{\max }} B_{m}^{k}(r)\left(\frac{d^{2}}{d r^{2}}-\frac{\ell(\ell+1)}{r^{2}}\right) B_{n}^{k}(r) d r
$$

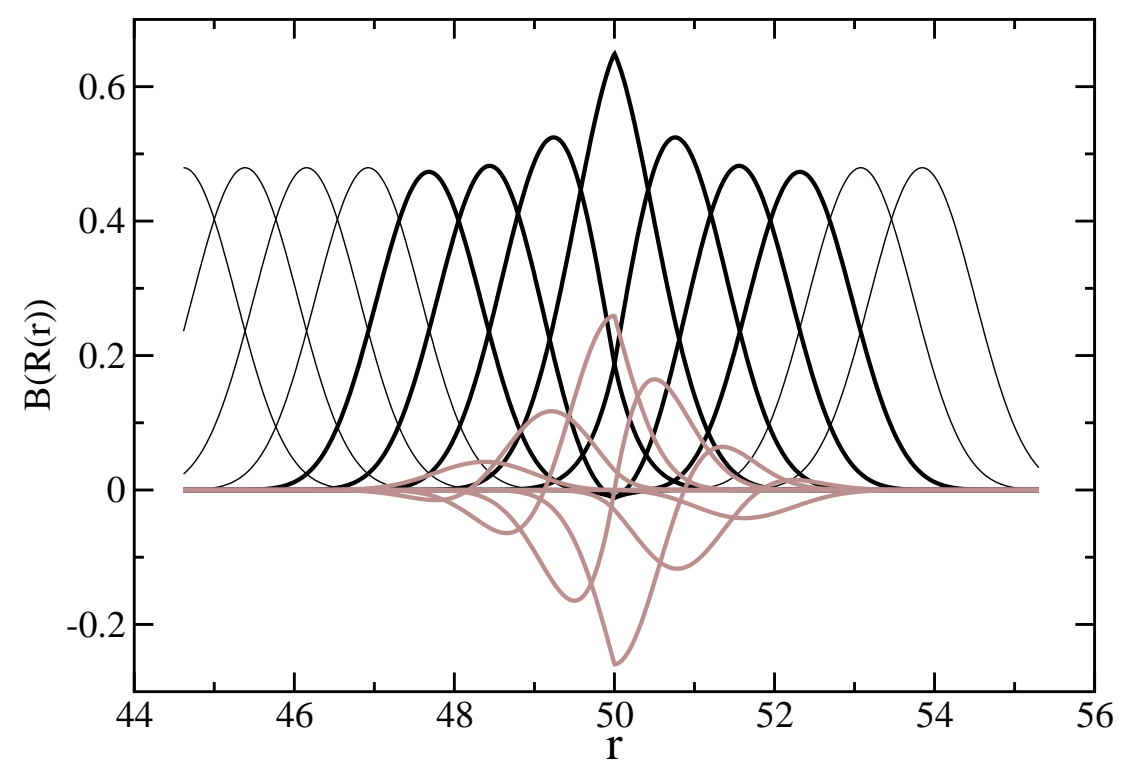

Figure 4. 8th order B-splines on the complex exterior scaling contour with $R_{0}=50$ and $\eta=40^{\circ}$. Heavy black lines are the real parts of the only complex splines, and thin black lines are the real B-splines. Grey lines are the imaginary parts. 


$$
=\quad \sum_{l} \int_{\xi_{l}}^{\xi_{l+1}}\left(\frac{d B_{m}^{k}(r)}{d r} \frac{d B_{n}^{k}(r)}{d r}+B_{m}^{k}(r) \frac{\ell(\ell+1)}{r^{2}} B_{n}^{k}(r)\right) d r(756
$$

where $\xi_{1}=0$ and $\xi_{N}=R\left(r_{\max }\right)$ is the end of the complex exterior scaling contour. In each interval in these sums, the integrals may be performed, for example, with simple Gauss-Legendre quadrature, but only the integrals over intervals on which both Bsplines are nonzero are required. Each of the integrals appearing here is on a straight line, the ends of which may be real or complex . The Jacobian factor, $q(r)$, of Eq.(25) is automatically included because the limits of the integrals are explicitly complex on the complex part of the contour.

With these definitions of the matrix elements, we can construct the B-spline representation of any one-electron Hamiltonian. It should be noted that the Hamiltonian in the B-spline representation is a banded matrix, whose bandwidth depends on the order of the B-splines.

To calculate two-electron integrals, analogous to Eq.(53), over B-splines we can use the same strategy as in the case of the FEM-DVR. We can solve the corresponding Poisson equation, following exactly the same logic as in the section 3.2, this time expanding the solution in B-splines instead of DVR basis functions as described by McCurdy and Martín [48]. The final result is similar to that of Eq.(70),

$$
\left\langle\rho_{B}\left|\frac{r_{<}^{\ell}}{r_{>}^{\ell+1}}\right| \rho_{A}\right\rangle=(2 \ell+1) \sum_{m, n=1}^{M} U_{m}^{B} T_{m, n}^{-1} U_{n}^{A}+\frac{1}{r_{m a x}^{2+1}} Q_{\ell}^{A} Q_{\ell}^{B}
$$

where

$$
\begin{aligned}
U_{m}^{A} & =\int_{0}^{r_{\max }} B_{m}(r) \frac{1}{r} \rho_{A}(r) d r \\
U_{n}^{B} & =\int_{0}^{r_{\max }} B_{n}(r) \frac{1}{r} \rho_{B}(r) d r \\
Q_{\ell}^{A} & =\int_{0}^{r_{\max }} \rho_{A}(t) t^{\ell} d t \\
Q_{\ell}^{B} & =\int_{0}^{r_{\max }} \rho_{B}(t) t^{\ell} d t
\end{aligned}
$$

and we have supressed the superscript denoting the order of the B-splines. The densities can be simple products of B-spline basis functions

$$
\begin{aligned}
& \rho_{A}(r)=B_{i}(r) B_{k}(r) \\
& \rho_{B}(r)=B_{j}(r) B_{l}(r)
\end{aligned}
$$

In a calculation on an atomic or molecular system in B-splines we can also have the densities $\rho(r)$ as products of sums of B-splines, but in any case all the quantities in Eq. $(76 a)$ are calculated from quadratures involving B-splines.

The working formula for two-electron integrals calculated as matrix elements Bsplines, Eq.(76a), and that for two-electron integrals calculated as matrix elements of the FEM-DVR basis functions, Eq.(70) have exactly the same form, but with one important difference. The B-spline version involves one-dimensional integrals and is not diagonal in the indices $i, k$ and $j, l$. Instead it is banded in those indices. The DVR version does not involve integrals and is diagonal in the corresponding indices. The B-spline approach provides as many continuous derivatives as the order of the splines allows, and does so globally, in contrast to the FEM-DVR approach which does not 
enforce continuity of any derivatives at the finite element boundaries. In principal that property of the B-spline basis allows a more accurate and possibly more compact representation of the wave function. But that advantage comes at a cost, requiring considerably more effort in computing the two electron integrals.

\subsection{Direct numerical integration}

It is relatively straightforward to see how to solve a one-dimensional Schrödinger equation on the "sharp" ECS contour using direct numerical integration, and that was in fact done early in the history of exterior complex scaling by Turner and McCurdy [61]. However for a two-electron problem no method existed until recently for direct numerical integration in two-dimensions, which would be necessary for solving the ECS Schrödinger equation under the rectangular two-dimensional scaling shown in Fig. 1b that corresponds to the scaling domain used in the FEM-DVR and B-spline methods discussed above.

It is for that reason that the recent work of Bartlett and Stelbovics [62, 63] marks a remarkable step forward in the application of the "sharp" ECS approach discussed in previous sections of this review. These authors and their coworkers have devised a two-dimensional marching algorithm for direct numerical integration of the exterior scaled Schrödinger equation, and applied it to electron impact ionization of one-electron target atoms.

For example, in the simplest two-dimensional example they treated, the TemkinPoet (spherical average) model whose Hamiltonian was given in Eq.(10), we can write the driven Schrödinger in Eq. $(29 a)$ as

$$
\left(E+\frac{1}{2} \frac{\partial^{2}}{\partial r_{1}^{2}}+\frac{1}{2} \frac{\partial^{2}}{\partial r_{2}^{2}}+\frac{1}{\min \left(r_{1}, r_{2}\right)}\right) \psi^{s c}\left(r_{1}, r_{2}\right)=\chi\left(r_{1}, r_{2}\right)
$$

where the right hand side, $\chi\left(r_{1}, r_{2}\right)$ is $(H-E) \psi_{0}$ with the appropriate choice of initial state, $\psi_{0}=\sqrt{2 \pi} / k\left(\phi_{1 s}\left(r_{1}\right) \sin \left(k r_{2}\right) \pm \sin \left(k r_{1}\right) \phi_{1 s}\left(r_{2}\right)\right)$. Stelbovics and coworkers apply the two-dimensional exterior complex scaling in Fig. 1b to this equation, and then make a two-dimensional 3-point Numerov finite difference approximation to the kinetic energy operators, so that the "stencil" involves nine points in all, using the eight nearest-neighbor grid points [62,64]. This Numerov formula must be generalized for the unequal (complex) step sizes around $R_{0}$ in much the same spirit as the generalization of finite differences in Eqs.(42a,42b, and $42 c)$. Thus in direct numerical integration using finite difference, we see yet again that the derivative discontinuity at $R_{0}$ can be easily represented exactly by grid-based methods.

Based on the approach originally used by Poet [65] and exploited by Jones and Stelbovics [66], Eq.(78) can be represented in matrix form as

$$
\mathbf{A}^{(i)} \cdot \vec{\psi}^{(i-1)}+\mathbf{B}^{(i)} \cdot \vec{\psi}^{(i)}+\mathbf{C}^{(i)} \cdot \vec{\psi}^{(i+1)}=\vec{\chi}^{(i)}
$$

where the notation $\vec{\psi}^{(i)}$ denotes the $i$ th column of the wave function represented on the two-dimensional grid. The working propagation equation is derived from the rearrangement of Eq.(79) and is of the form

$$
\overrightarrow{\boldsymbol{\psi}}^{(i)}=\mathbf{D}^{(i)} \cdot \overrightarrow{\boldsymbol{\psi}}^{(i+1)}+\mathbf{E}^{(i)}
$$

The matrices in Eq.(79) and Eq.(80) are defined in references [62,66] and [65]. The propagation matrices $\mathbf{D}^{(i)}$ and $\mathbf{E}^{(i)}$ are given by an outward marching algorthim, and the wave function is then produced by a "backward pass" using Eq.(80). The 
boundary conditions on the wave function are that it vanish on the boundaries of the grid, as in any ECS calculation, and those boundary conditions are involved in both "passes", forward and backward, across the rectangular grid. The practical algorithm also involves making use of the symmetry (even or odd) with respect to interchanging $r_{1}$ and $r_{2}$ in the wave function.

Stelbovics and coworkers have applied this algorithm to the calculation of electron impact ionization of hydrogen and other one-electron atoms [64, 63], augmenting it with an efficient iterative algorithm for solving the coupled equations (in the angular momenta of the electrons) of the breakup problem in its full dimensionality. A particularly remarkable aspect of this approach is that, since it is a marching algorithm, it can be carried out to large values of $R_{0}$ thereby allowing calculations at energies very near the ionization threshold. Values of $R_{0}$ ranging from 400 to 1000 bohr radii have been used in calculations that definitively explore the threshold region of the Temkin-Poet problem with this method [62].

\section{Calculating amplitudes and cross sections}

Once the scattering wave function has been calculated, we come to the second major step of the ECS method, namely, deciding how to extract the detailed dynamical information it describes. One would not normally view this as a major issue, since in most standard methods, the asymptotic boundary conditions that define the dynamical quantities of interest are used in the generation of the wave function. But in the ECS method, where detailed specification of scattering boundary conditions is avoided by design, what is obtained is a numerical representation of a wave function that contains information about all processes that are allowed at a specific total energy. Consider, for example, Fig. 5, which shows one radial component of the full wave function for the e- $\mathrm{H}$ system at a total energy of $4 \mathrm{eV}$ above the ionization threshold. Although it is possible to visually identify the spherical wavefronts that describe ionization and the structures along the two axes that correspond to elastic scattering and Rydberg state excitation, the numerical representation on a finite volume gives no immediate hints about how to evaluate the corresponding cross sections.

In the first implementations of ECS to e-H ionization [51], this problem was solved by simply computing the quantum mechanical flux,

$$
\mathbf{F}_{\rho}\left(\hat{r}_{1}, \hat{r}_{2}, \Omega\right)=(1 / 2 i)\left(\Psi_{s c}^{*} \nabla \Psi_{s c}-\Psi_{s c} \nabla \Psi_{s c}^{*}\right),
$$

through a surface that lies inside the region where the coordinates are real, and by extrapolating the result to infinite grid size, where the flux can be related to the differential cross sections for ionization. Aside from the problem of requiring fairly large grids, where one can be assured that $\mathbf{F}_{\rho}=\mathbf{F}_{\infty}+O(1 / \rho)$ [52], there are other intrinsic problems with this approach. The method requires that the grids be large enough to allow the physical region inhabited only by the ionization portion of the scattered wave to be distinguishable from the parts that describe discrete twobody channels. The requirement that the ionization wave be "uncovered" before the asymptotic flux is calculated can require grids that extend well beyond the range where the interaction potentials are appreciable. Moreover, the flux-extrapolation procedure is inherently limited in its ability to describe ionization when a single electron carries most of the available energy. This is due to its inability to distinguish beween excitation channels and ionization where energy is shared asymmetrically. In 


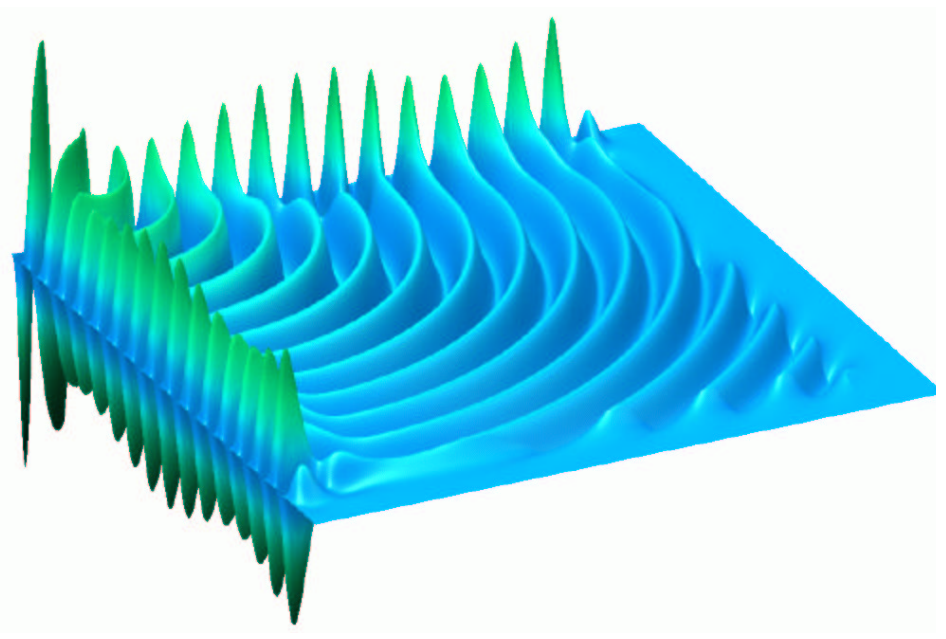

Figure 5. Real part of a representative radial function for electron-hydrogen scattering at $17.6 \mathrm{eV}$ incident energy. Vertical axis is $\operatorname{Re}(\Psi)$ and the two horizontal axes are $r_{1}$ and $r_{2}$ with origins at the rear left corner. $\Psi_{l_{1}, l_{2}}^{L}\left(r_{1}, r_{2}\right)$ is shown for singlet spin, $L=2$ and $l_{1}=l_{2}=1$

principle, these limitations could be made arbitrarily small by increasing the size of the calculation, but in practice the method falls short of being truly complete.

A far more practical approach to calculating breakup cross sections is to formulate the problem in terms of integral expressions for the underlying scattering amplitudes. Integral expressions for breakup amplitudes pose particular formal difficulties in the case of long-range forces. They can also present practical difficulties when evaluated on finite volumes, as they must be when using the finite grid ECS methods that are the subject of this review. An important aspect of the success of ECS approach is the fact that those difficulties can all be easily overcome. In the following sections we turn to the derivation of practical and compact integral expressions for breakup amplitudes involving two electrons in the continuum.

\subsection{Amplitudes for breakup by electron impact}

Integral expressions have frequently been employed for electron impact ionization studies in connection with various perturbative schemes such as the distorted wave Born approximation and other treatments that use ansatz three-body wave functions. These schemes usually begin with the 'prior' form of the amplitude and then develop approximations for $\Psi_{k_{1}, k_{2}}^{-}[6]$. We choose instead to begin with the 'post' form of the amplitude since ECS gives us a numerical representation of the outgoing portion of $\Psi^{+}$. In following this latter path, we have to address certain difficulties posed by the formal theory $[1,2,3]$ with regard to the proper choice of the unperturbed final state. These formal difficulties arise when the Coulomb forces are taken to infinity.

Since we are always working on a finite volume, however, we can safely begin the standard theory of rearrangement scattering [47], which is valid when only short-range interactions are present, by assuming the Coulomb potentials are truncated at a large but finite distance. We will return to the issue of reconciling our treatment with the formal theory in section 6 . 
We thus start with a formal expression for the breakup amplitude for the case of short-range interactions,

$$
\begin{aligned}
f\left(\mathbf{k}_{1}, \mathbf{k}_{2}\right) & =\left\langle\mathbf{k}_{1}, \mathbf{k}_{2}\left|V+V(E-H+i \epsilon)^{-1} V_{1}\right| \Psi_{0}\right\rangle_{\rho_{0}} \\
& \equiv\left\langle\mathbf{k}_{1}, \mathbf{k}_{2}\left|V+V G^{+}(E) V_{1}\right| \Psi_{0}\right\rangle_{\rho_{0}},
\end{aligned}
$$

where the final state is simply a product of plane waves $(\langle\mathbf{r} \mid \mathbf{k}\rangle=\exp (i \mathbf{k} \cdot \mathbf{r}))$ and $\Psi_{0}$ is the unperturbed initial state, i.e. the product of a plane wave for the incident electron and the initial target state. It is to be understood that the matrix element is carried out over a large, but finite volume defined by some hyperradius, $\rho_{0}$. All of the integrals in this section are performed on that finite volume, so we henceforth omit the $\rho_{0}$ subscript. The potential $V$ is the full interaction potiential, ie. $H=T+V$, while $V_{1}$ is the interaction potential appropriate to the initial channel:

$$
V_{1}\left|\Psi_{0}\right\rangle=(H-E)\left|\Psi_{0}\right\rangle
$$

so that

$$
\begin{aligned}
f\left(\mathbf{k}_{1}, \mathbf{k}_{2}\right) & =\left\langle\mathbf{k}_{1}, \mathbf{k}_{2}\left|V\left[1+G^{+}(E)(H-E)\right]\right| \Psi_{0}\right\rangle \\
& =\left\langle\mathbf{k}_{1}, \mathbf{k}_{2}|V| \Psi^{+}\right\rangle \\
& =\left\langle\mathbf{k}_{1}, \mathbf{k}_{2}|E-T| \Psi^{+}\right\rangle
\end{aligned}
$$

where we have used the fact that $(H-E) \Psi^{+}=0$. If we further partition $\Psi^{+}$into an incident and a scattered wave term, as in Eq.(28),

$$
\Psi^{+}=\Psi_{0}+\Psi^{s c}
$$

then it is easy to see that the contribution from the incident term makes no contribution to Eq.(84), since it is proportional to $\delta$ functions between the incident and final momenta, which cannot be the same. So we have,

$$
\begin{aligned}
f\left(\mathbf{k}_{1}, \mathbf{k}_{2}\right) & =\left\langle\mathbf{k}_{1}, \mathbf{k}_{2}|E-T| \Psi^{s c}\right\rangle \\
& =\left\langle\mathbf{k}_{1}, \mathbf{k}_{2}|E-T| G^{+}(H-E) \Psi_{0}\right\rangle .
\end{aligned}
$$

Eq.(86), while formally correct, gives spurious results when evaluated on a finite volume, as we have previously shown [67]. These arise from the parts of $\Psi^{s c}$ that describe discrete, two-body channels. Their contributions, for an infinite volume, become proportional to $\delta$ functions between initial and final momenta and must therefore vanish. On a finite volume, they produce spurious contributions that render Eq.(86) computationally unstable.

We can eliminate this problem by using distorted waves to replace the plane wave final states. If the distorted waves are chosen to be eigenfunctions of the target Hamiltonian, then there will be no spurious two-body contributions to the ionization amplitude because the distorted waves and the discrete target states are orthogonal. The derivation of the working equations uses the standard theory of rearrangement scattering. We begin by writing the full interaction as the sum of some one-body distorted-wave potential, $V_{d}$, and a remainder:

$$
\begin{aligned}
& V_{d}=v_{d}\left(\mathbf{r}_{1}\right)+v_{d}\left(\mathbf{r}_{2}\right) \\
& V=V_{d}+\Delta V,
\end{aligned}
$$

and use the two-potential formula [47] to express the full Green's function in terms of, $g_{d}^{+}$, the Green's function for $V_{d}$ :

$$
G^{+}(E)=g_{d}^{+}(E)+g_{d}^{+}(E) \Delta V G^{+}(E)
$$


Using the above relationship along with the identity

$$
(E-T) G^{+}(E)=1+V G^{+}(E),
$$

we can rearrange Eq.(86) to read:

$$
f\left(\mathbf{k}_{1}, \mathbf{k}_{2}\right)=\left\langle\mathbf{k}_{1}, \mathbf{k}_{2} \mid\left[1+V_{d} g_{d}^{+}(E)\right]\left[1+\Delta V G^{+}(E)\right](H-E) \Psi_{0}\right\rangle .
$$

But $\left[1+V_{d} g_{d}^{+}(E)\right]$ is the wave operator for the potential $V_{d}$ which converts the product $\left|\mathbf{k}_{1}, \mathbf{k}_{2}\right\rangle$ into a product of distorted waves $\left|\Phi_{\mathbf{k}_{1}}^{d+}, \Phi_{\mathbf{k}_{2}}^{d+}\right\rangle$. We thus arrive at the "twopotential" formula for the amplitude:

$$
f\left(\mathbf{k}_{1}, \mathbf{k}_{2}\right)=\left\langle\Phi_{\mathbf{k}_{1}}^{d-}, \Phi_{\mathbf{k}_{2}}^{d-}|(H-E)| \Psi_{0}\right\rangle+\left\langle\Phi_{\mathbf{k}_{1}}^{d-}, \Phi_{\mathbf{k}_{2}}^{d-}|\Delta V| \Psi^{s c}\right\rangle .
$$

This can be further simplified by using the the original driven Schrödinger equation that defines $\Psi^{s c}$,

$$
(E-H) \Psi^{s c}=(H-E) \Psi_{0},
$$

to obtain

$$
\begin{aligned}
f\left(\mathbf{k}_{1}, \mathbf{k}_{2}\right) & =\left\langle\Phi_{\mathbf{k}_{1}}^{d-}, \Phi_{\mathbf{k}_{2}}^{d-}|(E-H)| \Psi^{s c}\right\rangle+\left\langle\Phi_{\mathbf{k}_{1}}^{d-}, \Phi_{\mathbf{k}_{2}}^{d-}|\Delta V| \Psi^{s c}\right\rangle \\
& =\left\langle\Phi_{\mathbf{k}_{1}}^{d-}, \Phi_{\mathbf{k}_{2}}^{d-}\left|\left(E-T-V_{d}\right)\right| \Psi^{s c}\right\rangle .
\end{aligned}
$$

Finally, we can use Green's theorem to express Eq.(93) in terms of a matrix element with the kinetic energy operating to the left. But since the final state is an eigenfunction of the $\left(T+V_{d}\right)$ with energy $E$, only the surface term survives and we get,

$$
f\left(\mathbf{k}_{1}, \mathbf{k}_{2}\right)=\int_{S}\left(\Phi_{\mathbf{k}_{1}}^{d-*} \Phi_{\mathbf{k}_{2}}^{d-*} \nabla \Psi^{s c}-\Psi^{s c} \nabla \Phi_{\mathbf{k}_{1}}^{d-*} \Phi_{\mathbf{k}_{2}}^{d-*}\right) \cdot d \hat{\mathbf{S}}
$$

Thus the amplitude is determined solely by the asymptotic behavior of $\Psi^{s c}$ at $\rho=\rho_{0}$.

The derivation above is formally valid for any choice of one-body distorted-wave potential. The choice that removes the spurious behavior alluded to above and that gives stable results for the e- $\mathrm{H}$ problem $[68,69,70]$ is $V_{d i s}=V_{1}=-1 / r_{1}-1 / r_{2}$. With this choice, the distorted waves are simply Coulomb functions with charge $Z=-1$.

\subsection{Amplitudes for inelastic scattering}

The amplitudes for excitation of discrete final states can also be extracted from the scattered wave function via surface integrals analogous to those derived for breakup, as originally outined by McCurdy, Horner and Rescigno [71]. We begin with the usual expression for the excitation amplitude:

$$
\begin{aligned}
f_{0 \rightarrow n} & =\left\langle\mathbf{k}_{n}, \Phi_{n}\left|V_{1}\right| \Psi^{+}\right\rangle \\
& =\left\langle\mathbf{k}_{n}, \Phi_{n}\left|H_{1}-E\right| \Psi^{+}\right\rangle,
\end{aligned}
$$

where $H_{1}=T+V_{1}, \mathbf{k}_{n}$ is a free (plane-wave) state and $\Phi_{n}$ is a discrete target state. We can again use Green's theorem, along with the fact that $\left(H_{1}-E\right)\left|\mathbf{k}_{n}, \Phi_{n}\right\rangle=0$, to express Eq.(95) as a surface integral:

$$
\begin{aligned}
f_{0 \rightarrow n} & =\int_{S}\left(\mathbf{k}_{n} \Phi_{n} \nabla \Psi^{+}-\Psi^{+} \nabla \mathbf{k}_{n} \Phi_{n}\right) \cdot d \hat{\mathbf{S}} \\
& =\int_{S}\left(\mathbf{k}_{n} \Phi_{n} \nabla \Psi^{s c}-\Psi^{s c} \nabla \mathbf{k}_{n} \Phi_{n}\right) \cdot d \hat{\mathbf{S}} .
\end{aligned}
$$

Note that the replacement of $\Psi^{+}$by $\Psi^{s c}$ in the surface integral follows from the fact that the discrete target states vanish at large $\rho$.

Bartlett et al. [64, 62], evidently unaware of our earlier exposition [71], rederived Eq.(96) and used it to compute excitation cross sections in their recent implementation of ECS. 


\subsection{Amplitudes for double photoionization}

Atomic double photoionization is a "half-collision" process in which an atom absorbs a single photon and ejects two electrons into the continuum. Like electron-impact ionization, the problem is one in which the final state involves three unbound charged particles, so much of the formalism needed to describe collisional ionization pertains to double photoionization as well.

In the weak field limit, the amplitude that describes this process is a matrix element of the dipole operator between the initial target wave function and a final state involving a doubly charged ion and two free electrons, which in the "velocity form" is,

$$
\mathfrak{F}\left(\mathbf{k}_{1}, \mathbf{k}_{2}\right)=\left\langle\Psi_{k_{1}, k_{2}}^{-}\left|\epsilon \cdot\left(\nabla_{1}+\nabla_{2}\right)\right| \Gamma_{0}\right\rangle
$$

where $\epsilon$ is the polarization unit vector, and $\left|\Gamma_{0}\right\rangle$ is the initial (bound) state of the atom. In Eq.(97) the required final state wave function, $\Psi_{k_{1}, k_{2}}^{-}$, whose initial channel is one where both electrons are in the continuum, is the same wave function we referred to in section 1.1, and in section 4.1 when we discussed the "prior" form of the collisional breakup amplitude. Following this route would require us to solve a driven Schrödinger equation for each energy sharing of final state photo-electrons we were interested in.

A more efficient path is to begin with the first-order equation that describes singlephoton absorption by a 2 -electron atom, which we can write, again in the velocity form as:

$$
\left(E_{0}+\omega-H\right)\left|\Psi_{1}^{+}\right\rangle=\epsilon \cdot\left(\nabla_{1}+\nabla_{2}\right)\left|\Gamma_{0}\right\rangle=\left(\frac{\partial}{\partial z_{1}}+\frac{\partial}{\partial z_{2}}\right)\left|\Gamma_{0}\right\rangle
$$

where we have chosen the $z$ axis along the polarization vector.

This driven Schrödinger equation has the same structure as the scattered wave equation we considered in the (e,2e) case, with the dipole operator times the initial state replacing $(H-E) \Psi_{0}$ as the driving term. The asymptotic form of the solution of this equation can therefore be analyzed following the same line of reasoning employed by Rudge [3] in his formal analysis of the electron-impact ionization problem:

$$
\Psi_{1}^{+} \rightarrow(2 \pi i)^{1 / 2} \chi\left(\mathbf{k}_{1}, \mathbf{k}_{2}, \rho\right)\left(\frac{K^{3}}{\rho^{5}}\right)^{1 / 2} \mathcal{F}\left(\hat{r}_{1}, \hat{r}_{2}, \alpha\right) e^{i K \rho+i \frac{\zeta}{K} \ln (2 K \rho)}
$$

where $\mathcal{F}$ is proportional to the double photoionization amplitude and the quantities $\rho$, $\alpha, K$ and $\zeta$ are defined as in Eq.(2). In Eq.(99) $\chi\left(\mathbf{k}_{1}, \mathbf{k}_{2}, \rho\right)$ is an (irrelevant) overall phase factor, which can be analyzed $[58,72]$ as in the discussion in section 6 below, and which contains the "Peterkop phase" that depends logarithmically on $\rho$.

We can develop an integral expression for the double photoionization amplitude in the same way we did for the breakup amplitude in section 4.1. The resulting expression for the amplitude we derive is [58]:

$$
\begin{aligned}
\mathcal{F}\left(\hat{r}_{1}, \hat{r}_{2}, \alpha\right) & =\frac{2}{\pi}\left\langle\Phi_{\mathbf{k}_{1}}^{-}, \Phi_{\mathbf{k}_{2}}^{-}\left|\left(E-T-V_{1}\right)\right| \Psi_{1}^{+}\right\rangle \\
& =\frac{1}{\pi} \int_{S}\left(\Phi_{\mathbf{k}_{1}}^{-*} \Phi_{\mathbf{k}_{2}}^{-*} \nabla \Psi_{1}^{+}-\Psi_{1}^{+} \nabla \Phi_{\mathbf{k}_{1}}^{-*} \Phi_{\mathbf{k}_{2}}^{-*}\right) \cdot d \hat{\mathbf{S}}
\end{aligned}
$$

where $\Phi_{\mathbf{k}}^{-}$is a Coulomb function with charge $Z$, normalized as in Eq.(106), and $V_{1}$ is the one-body part of the interaction potential,

$$
V_{1}=-Z / r_{1}-Z / r_{2}
$$

with $Z$ the nuclear charge of the atom. 


\section{Partial wave analysis of breakup amplitudes}

In a practical calculation on either electron-impact ionization or double photoionization of an atom, we exploit the spherical symmetry of the target in a partial wave analysis. The integral expressions for breakup amplitudes in Section 4 and their corresponding surface integral representations then reduce to combinations of amplitudes computed on an arc in the $\left\{r_{1}, r_{2}\right\}$ plane of radial coordinates. That partial wave analysis is the subject of this section.

\subsection{Electron-impact ionization}

In carrying our numerical computations for ionization by electron impact, we solve the driven Schrödinger equation

$$
(E-H) \Psi^{s c}\left(\mathbf{r}_{1}, \mathbf{r}_{2}\right)=(H-E) \Psi_{0}\left(\mathbf{r}_{1}, \mathbf{r}_{2}\right)
$$

by adopting a convential partial-wave description and expanding both $\Psi_{0}$ and $\Psi^{s c}$ in coupled spherical harmonics $y l_{l_{1} l_{2}}^{L M}$,

$$
y_{l_{1} l_{2}}^{L M}\left(\hat{\mathbf{r}}_{1}, \hat{\mathbf{r}}_{2}\right)=\sum_{m_{1}, m_{2}}\left(l_{1} m_{1} l_{2} m_{2} \mid l_{1} l_{2} L M\right) Y_{l_{1}, m_{1}}\left(\hat{\mathbf{r}}_{1}\right) Y_{l_{2}, m_{2}}\left(\hat{\mathbf{r}}_{2}\right)
$$

using the notation of Edmonds [73] for the vector coupling coefficients. For example, for ionization of the hydrogen atom in its $1 s$ ground-state, we write

$$
\begin{aligned}
\Psi_{0} & =\frac{1}{\sqrt{2}}\left[\Phi_{1 s}\left(\mathbf{r}_{1}\right) e^{i \mathbf{k}_{\mathbf{i}} \cdot \mathbf{r}_{2}}+(-1)^{S} \Phi_{1 s}\left(\mathbf{r}_{2}\right) e^{i \mathbf{k}_{\mathbf{i}} \cdot \mathbf{r}_{1}}\right] \\
& =\sum_{L=0}^{\infty} \frac{i^{L} \sqrt{2 \pi(2 L+1)}}{r_{1} r_{2} k_{i}}\left[\phi_{1 s}\left(r_{1}\right) j_{L}\left(k_{i} r_{2}\right) y_{0 L}^{L 0}\left(\hat{\mathbf{r}}_{1}, \hat{\mathbf{r}}_{2}\right)\right. \\
& \left.+(-1)^{S} \phi_{1 s}\left(r_{2}\right) j_{L}\left(k_{i} r_{1}\right) y_{L 0}^{L 0}\left(\hat{\mathbf{r}}_{1}, \hat{\mathbf{r}}_{2}\right)\right]
\end{aligned}
$$

and

$$
\Psi^{s c}=\sum_{L, l_{1}, l_{2}} \frac{i^{L}}{r_{1} r_{2}} \psi_{l_{1} l_{2}}^{L}\left(r_{1}, r_{2}\right) y_{l_{1} l_{2}}^{L 0}\left(\hat{\mathbf{r}}_{1}, \hat{\mathbf{r}}_{2}\right),
$$

and solve coupled two-dimensional equations for the radial functions $\psi_{l_{1} l_{2}}^{L}\left(r_{1}, r_{2}\right)$. To evaluate the ionization amplitude using the surface integral, Eq.(94), we also need the partial wave expansion of the Coulomb function:

$$
\Phi_{\mathbf{k}}^{-}(\mathbf{r})=\sum_{m=-l}^{l} \frac{i^{l} e^{-i \eta_{l}(k)}}{k r} \varphi_{l}^{(c)}(k, r) Y_{l, m}^{*}(\hat{\mathbf{k}}) Y_{l, m}(\mathbf{r})
$$

where the radial Coulomb function, $\varphi_{k l}^{(c)}(r)$, has the asymptotic normalization

$$
\varphi_{k l}^{(c)}(r) \rightarrow \sin \left(k r+\frac{Z}{k} \ln 2 k r-\frac{\pi l}{2}+\eta_{l}(k)\right),
$$

with

$$
\eta_{l}(k)=\arg \Gamma(l+1-i / k) .
$$

If we substitute Eqs. (105) and (106) into Eq.(94), we get the following expression for the ionization amplitude:

$$
f\left(\mathbf{k}_{1}, \mathbf{k}_{2}\right)=\sum_{L, l_{1}, l_{2}} i^{L-l_{1}-l_{2}} e^{i\left(\eta_{l_{1}}\left(k_{1}\right)+\eta_{l_{2}}\left(k_{2}\right)\right)} f_{l_{1}, l_{2}}^{L}\left(k_{1}, k_{2}\right) y_{l_{1} l_{2}}^{L 0}\left(\hat{\mathbf{k}}_{1}, \hat{\mathbf{k}}_{2}\right),
$$


where

$$
\begin{aligned}
f_{l_{1}, l_{2}}^{L}\left(k_{1}, k_{2} ; \rho_{0}\right)= & \\
& \frac{\rho_{0}}{2 k_{1} k_{2}} \int_{0}^{\pi / 2} d \alpha\left(\varphi_{l_{1}}^{(c)}\left(k_{1}, r_{1}\right) \varphi_{l_{2}}^{(c)}\left(k_{2}, r_{2}\right) \frac{d}{d \rho} \psi_{l_{1}, l_{2}}^{L}-\right. \\
& \left.\psi_{l_{1}, l_{2}}^{L} \frac{d}{d \rho}\left[\varphi_{l_{1}}^{(c)}\left(k_{1}, r_{1}\right) \varphi_{l_{2}}^{(c)}\left(k_{2}, r_{2}\right)\right]\right)\left.\right|_{\rho=\rho_{0}} .
\end{aligned}
$$

The triple differential cross section (TDCS), which is differential in energy sharing and the directions of both outgoing electrons, is obtained directly from $f\left(\mathbf{k}_{1}, \mathbf{k}_{2}\right)$ by the expression

$$
\frac{d^{3} \sigma}{d E_{1} d \Omega_{1} d \Omega_{2}}=\frac{16 \pi^{2} k_{1} k_{2}}{k_{i}^{2}}\left|f\left(\mathbf{k}_{1}, \mathbf{k}_{2}\right)\right|^{2}
$$

where $\Omega_{1}$ and $\Omega_{2}$ are the solid angles associated with $\mathbf{k}_{1}$ and $\mathbf{k}_{2}$. The double differential cross section (DDCS) measures the energy and direction of only one of the ejected electrons and is obtained by integrating TDCS over one set of solid angles. The single differential or energy sharing cross section (SDCS) is defined as the integral of the TDCS over $\Omega_{1}$ and $\Omega_{2}$ and, because of the orthogonality properies of the coupled spherical harmonics, is simply

$$
\frac{d \sigma}{d E_{1}}=\frac{16 \pi^{2} k_{1} k_{2}}{k_{i}^{2}} \sum_{L, l_{1}, l_{2}}\left|f_{l_{1}, l_{2}}^{L}\left(k_{1}, k_{2}\right)\right|^{2}
$$

where the total energy is $E=k_{1}^{2} / 2+k_{2}^{2} / 2=E_{1}+E_{2}$. The total ionization cross section (TICS) is then

$$
\sigma_{\text {ion }}=\int_{0}^{E} \frac{d \sigma}{d E_{1}} d E_{1}
$$

although the TICS is frequently defined as the integral over half this interval, which requires a definition of the single differential cross section as

$$
\frac{d \tilde{\sigma}}{d E_{1}}=2 \frac{d \sigma}{d E_{1}}
$$

so that

$$
\sigma_{i o n}=\int_{0}^{E / 2} \frac{d \tilde{\sigma}}{d E_{1}} d E_{1}
$$

\subsection{Double photoionization}

The decomposition of the double photoionization amplitude into partial-waves can be carried out in complete analogy with the analysis of the electron impact ionization amplitude given in section 5 . We use a representation of the solution of the first-order equation, $\Psi_{1}^{+}$, in Eq.(98) in coupled spherical harmonics:

$$
\Psi_{1}^{+}\left(\mathbf{r}_{1}, \mathbf{r}_{2}\right)=\sum_{l_{1}, l_{2}} \frac{1}{r_{1} r_{2}} \psi_{l_{1} l_{2}}^{L}\left(r_{1}, r_{2}\right) y_{l_{1} l_{2}}^{L M}\left(\hat{\mathbf{r}}_{1}, \hat{\mathbf{r}}_{2}\right)
$$

Note that, in contrast to the electron impact case, we need only retain a one $L$ component in the expansion because the dipole operator that appears on the RHS of Eq.(98) imposes specific selection rules. So, for example, to treat double photoionization of ground-state helium, we need only compute the $L=1$ component 
of the scattered wave. The rest of the analysis proceeds exactly as that given in section 5.1 , so for the ionization amplitude, we obtain the expression

$\mathcal{F}\left(\mathbf{k}_{1}, \mathbf{k}_{2}\right)=\sum_{l_{1}, l_{2}} i^{-l_{1}-l_{2}} e^{i\left(\eta_{l_{1}}\left(k_{1}\right)+\eta_{l_{2}}\left(k_{2}\right)\right)} \mathcal{F}_{l_{1}, l_{2}}^{L}\left(k_{1}, k_{2}\right) y_{l_{1} l_{2}}^{L M}\left(\hat{\mathbf{k}}_{1}, \hat{\mathbf{k}}_{2}\right)$,

with

$\mathcal{F}_{l_{1}, l_{2}}^{L}\left(k_{1}, k_{2} ; \rho_{0}\right)=$

$$
\begin{aligned}
& \frac{\rho_{0}}{\pi k_{1} k_{2}} \int_{0}^{\pi / 2} d \alpha\left(\varphi_{l_{1}}^{(c)}\left(k_{1}, r_{1}\right) \varphi_{l_{2}}^{(c)}\left(k_{2}, r_{2}\right) \frac{d}{d \rho} \psi_{l_{1}, l_{2}}^{L}-\right. \\
& \left.\psi_{l_{1}, l_{2}}^{L} \frac{d}{d \rho}\left[\varphi_{l_{1}}^{(c)}\left(k_{1}, r_{1}\right) \varphi_{l_{2}}^{(c)}\left(k_{2}, r_{2}\right)\right]\right)\left.\right|_{\rho=\rho_{0}} .
\end{aligned}
$$

For the helium case, the $\varphi_{l}^{(c)}$ are partial-wave Coulomb functions with charge $Z=2$.

The TDCS for double photoionization is the given by

$$
\frac{d^{3} \sigma}{d E_{1} d \Omega_{1} d \Omega_{2}}=\frac{4 \pi^{2}}{\omega c} k_{1} k_{2}\left|\mathcal{F}\left(\mathbf{k}_{1}, \mathbf{k}_{2}\right)\right|^{2}
$$

and the corresponding SDCS is

$$
\frac{d \sigma}{d E_{1}}=\frac{4 \pi^{2}}{\omega c} k_{1} k_{2} \sum_{l_{1} l_{2}}\left(\left|\mathcal{F}_{l_{1} l_{2} k_{1} k_{2}}\right|^{2}\right)
$$

If one chooses to use an explicitly symmetrized expansion of the scattered wave, as we did in our study of helium double photoionization using complex-scaled B-splines [58], it may be convenient to decompose the full ionization amplitude into direct and exchange partial wave amplitudes. Such an analysis is carried out in ref. [58].

\section{The overall phase of the breakup amplitude in the formal theory of ionization}

We have remarked at the outset that the formal theory of ionization has not yet provided a practical path for first-principles computation of the full wave function. On the other hand, it is interesting to reverse the argument and to ask whether a wave function obtained from an $a b$ initio calculation can be interrogated in a way to see if it has characteristics demanded by the formal theory. One way to test this is to examine the phase of the computed ionization amplitude. It turns out that this phase can be used to make contact with the formal theory and, in addition, provides a very sensitive test for gauging numerical convergence.

Issues related to the proper definition of the phase of the ionization amplitude have been discussed in several recent formal studies [10, 74]. Moreover, there appears to have been some confusion about the way the phase of the ionization amplitude is handled in the ECS formalism [75], which in turn prompted us to examine the matter in more detail [72].

To illustrate the discussion, it suffices [72] to consider any two-dimensional (ie. zero angular momentum) model of e-H ionization, described by a radial Schrödinger equation:

$$
\left[-\frac{1}{2} \frac{\partial^{2}}{\partial r_{1}^{2}}-\frac{1}{2} \frac{\partial^{2}}{\partial r_{2}^{2}}-\frac{1}{r_{1}}-\frac{1}{r_{2}}+V_{2}\right] \psi=E \psi
$$


where $V_{2}$ is the (model) two-body interaction and $E=K^{2} / 2$ is the total energy. In hyperspherical coordinates $\left(\rho=\sqrt{r_{1}^{2}+r_{2}^{2}}, \tan (\alpha)=r_{2} / r_{1}\right)$, Eq.(121) becomes, with $\psi=\phi / \rho^{1 / 2}$

$$
\left[-\frac{1}{2} \frac{\partial^{2}}{\partial \rho^{2}}-\frac{1}{8 \rho^{2}}-\frac{1}{2 \rho^{2}} \frac{\partial^{2}}{\partial \alpha^{2}}-\frac{\zeta(\alpha)}{\rho}\right] \phi=E \phi,
$$

where $\zeta(\alpha)$ is defined by the full interaction potential:

$$
-\frac{1}{r_{1}}-\frac{1}{r_{2}}+V_{2}=-\frac{\zeta(\alpha)}{\rho} \text {. }
$$

Following Rudge's approach[3] for the full ionization problem, we rearrange the Schrödinger equation as

$$
\left[\frac{K^{2}}{2}+\frac{1}{2} \frac{\partial^{2}}{\partial \rho^{2}}+\frac{1}{8 \rho^{2}}+\frac{\zeta(\alpha)}{\rho}\right] \phi=-\frac{1}{2 \rho^{2}} \frac{\partial^{2}}{\partial \alpha^{2}} \phi,
$$

and formally solve this using the Coulomb Green's function, $G\left(\rho, \rho^{\prime}\right)$, with $\alpha$ as a parameter on the left-hand side. The Green's function for Eq.(124) is given by

$$
G\left(\rho, \rho^{\prime}\right)=-\frac{2}{K} F\left(\zeta, K, \rho_{\zeta}\right) H\left(\zeta, K, \rho_{\rangle}\right),
$$

with

$$
\begin{array}{lr}
F(\zeta, K, \rho) \sim \sin (f(\rho)) & \rho \rightarrow \infty \\
H(\zeta, K, \rho) \sim \exp (i f(\rho)) & \rho \rightarrow \infty
\end{array}
$$

It immediately follows [72] that the asymptotic form of the wave function can be written

$$
\begin{aligned}
\psi\left(r_{1}, r_{2}\right)= & -\frac{1}{\sqrt{\rho}} \int_{0}^{\infty} d \rho^{\prime} G\left(\rho, \rho^{\prime}\right) \frac{1}{2 \rho^{2}} \frac{\partial^{2}}{\partial \alpha^{2}} \phi\left(\rho^{\prime}, \alpha\right) \\
& \sim \frac{A(\alpha)}{\sqrt{\rho}} \exp \left[i\left(K \rho+\frac{\zeta(\alpha)}{K} \ln (2 K \rho)\right)\right]
\end{aligned}
$$

with the ionization amplitude, $A(\alpha)$ identified as

$$
\begin{aligned}
& A(\alpha)=\frac{1}{K} \exp \left[i\left(\frac{\pi}{4}+\arg \Gamma\left(\frac{1}{2}-i \frac{\zeta(\alpha)}{K}\right)\right)\right] \\
& \int_{0}^{\infty} d \rho F(\zeta, K, \rho) \frac{1}{2 \rho^{2}} \frac{\partial^{2}}{\partial \alpha^{2}} \phi(\rho, \alpha) .
\end{aligned}
$$

Peterkop points out that this treatment only defines $A(\alpha)$ to within an $\alpha$ dependent phase, which is a fundamental shortcoming of the hyperspherical approach. Kadyrov et al. [10] have recently shown how to remove this amplitude-phase ambiguity by defining the ionization amplitude in terms of the plane-wave function $\Psi_{k_{1}, k_{2}}^{-}$(see Eq.(6)), ie. using the prior rather than the post definition of the amplitude. We will not concern ourselves further with these distinctions since the phase in question is an overall phase in the amplitude for a specific physical process, and as such does not change any physical observable.

We can now consider an integral expression for the amplitude of the form

$$
I\left(k_{1}, k_{2}\right)=\int \psi(H-E) \psi_{0} d r_{1} d r_{2}
$$


where, as in the formal theory, we choose $\psi_{0}$ to be the product of two radial Coulomb functions with effective charges $z_{1}$ and $z_{2}$ and momenta $k_{1}$ and $k_{2}$ defined as:

$$
\begin{aligned}
& k_{1}=K \cos (\beta) \\
& k_{2}=K \sin (\beta) .
\end{aligned}
$$

With these definitions, we have

$$
\begin{aligned}
& \psi_{0}\left(r_{1}, r_{2}\right)=\phi_{k_{1}}\left(r_{1} ; z_{1}\right) \phi_{k_{2}}\left(r_{2} ; z_{2}\right) \\
& \phi_{k}(r ; z) \sim \sin \left(k r+\frac{z}{k} \ln (2 k r)+\eta_{k}(z)\right), \quad r \rightarrow \infty
\end{aligned}
$$

where

$$
\eta_{k}(z)=\arg \Gamma\left(1-i \frac{z}{k}\right)
$$

To evaluate the integral, $I$, we again switch to hyperspherical coordinates and use Green's theorem to express it as a surface integral:

$$
I=-\frac{1}{2} \lim _{\rho \rightarrow \infty} \int_{0}^{\pi / 2}\left[\psi \frac{\partial \psi_{0}}{\partial \rho}-\psi_{0} \frac{\partial \psi}{\partial \rho}\right] \rho d \alpha
$$

To evaluate the surface integral, we substitute the asymptotic forms for $\psi$ and $\psi^{0}$ given in Eqs. (127) and (131), respectively. The integration can be carried out using the method of stationary phase [76], which is exact in the limit $\rho \rightarrow \infty$. The algebra involved is tedious, but straightforward, and is outlined in ref. [72]. The result is

$$
\begin{aligned}
& I \underset{\lim \rho \rightarrow \infty}{=}-\frac{1}{4} \sqrt{2 \pi K} i A(\beta) \\
& \quad \times \exp \left[-i\left(Q\left(k_{1}, z_{1}\right)+Q\left(k_{2}, z_{2}\right)+\frac{3 \pi}{4}\right)\right] \\
& \quad \times \exp [i \phi \ln (2 K \rho)]+O(1 / \rho),
\end{aligned}
$$

with

$$
Q(k ; z)=\eta_{k}(z)+2 \frac{z}{k} \ln (k / K),
$$

and

$$
\phi=\frac{\zeta(\beta)}{K}-\frac{z_{1}}{k_{1}}-\frac{z_{2}}{k_{2}}
$$

We have remarked above that the hyperspherical approach only defines the ionization amplitude to within an arbitrary phase. Thus the factors in Eq.(134) involving $Q\left(k_{1}, z_{1}\right)$ and $Q\left(k_{2}, z_{2}\right)$ can easily be incorporated into the definition of the ionization amplitude, $A(\beta)$, with no physical consequences. The remaining portion of the phase that depends logarithmically on $\rho$ is more problematic, since it diverges when the volume of integration is taken to infinity, unless the effective charges are chosen to make $\phi$ vanish:

$$
\frac{\zeta(\beta)}{K}=\frac{z_{1}}{k_{1}}+\frac{z_{2}}{k_{2}}
$$

This is the so-called Peterkop condition [1].

We have already emphasized the fact that in numerical implementations of ECS it is essential to maintain orthogonality between the Coulomb distorted waves and the bound hydrogenic states and therefore remove any spurious contributions to the breakup amplitude that arise from discrete two-body excitation channels. This leads 

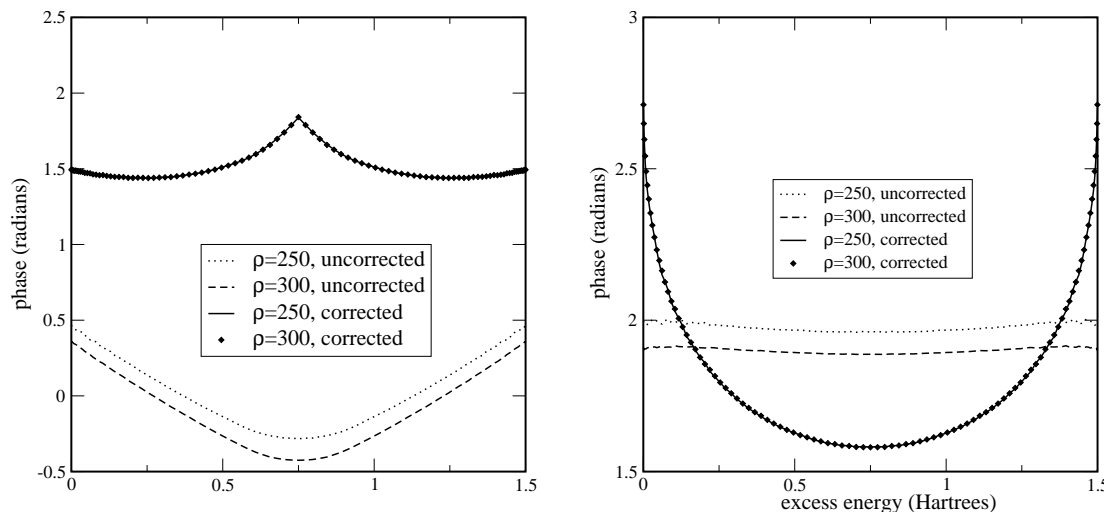

Figure 6. Phase of the computed amplitude for the single differential cross section for breakup at a total energy of 1.5 Hartree for different 2D models of e-H ionization. Left panel: Temkin-Poet model; right panel: collinear model. The curves labeled uncorrected were computed using Eq.(133) at a fixed value of the hyperradius. The corrected curves are obtained by removing the appropriate volume dependent portions of the phase for each model, as discussed in the text.

naturally to the choice $z_{1}=z_{2}=1$, as explained at length in refs. [67] and [68] a choice which generally does not satisfy the Peterkop condition and therefore leads to a divergent phase on an infinite volume. Divergence of the phase of the ionization amplitude is of course not a concern when the integral is carried out on a finite volume. Moreover, for any choice of effective charges, the computed amplitude should carry a predictable $\rho$-dependent "Peterkop phase", $\chi(\rho) \equiv \exp [i \phi \ln (2 K \rho)]$ - a fact that can easily be tested numerically. Such a test was carried out in ref. [72] for two popular 2D models of e-H ionization, the Temkin-Poet (TP) model and the collinear (CO) model, which approximate the electron-electron inteaction as:

$$
\begin{aligned}
V_{2}^{T P}\left(r_{1}, r_{2}\right) & =\frac{1}{\max \left(r_{1}, r_{2}\right)} \\
V_{2}^{C O}\left(r_{1}, r_{2}\right) & =\frac{1}{r_{1}+r_{2}}
\end{aligned}
$$

Using these definitions, along with Eqs. (123) and (136), we find that the choice $z_{1}=z_{2}=1$ produces the following Peterkop phases for these two cases:

$$
\begin{aligned}
& \chi^{T P}(\rho)=-1 / k_{>} \ln (2 K \rho) \\
& \chi^{C O}(\rho)=-1 /\left(k_{1}+k_{2}\right) \ln (2 K \rho) .
\end{aligned}
$$

Figure 6 shows numerical ECS results for the phases of the ionization amplitude from the TP and CO models, before and after removal of the Peterkop phase. Removing the Peterkop phase clearly gives a $\rho$-independent result.

For the two-dimensional models of ionization just considered, any overall phase carried by the breakup amplitude has no physical consequence, since it makes no contribution to the SDCS. For the full six-dimensional e-H problem, however, the above considerations are more than an idle curiosity. In the full problem, one typically decomposes the wave function into total angular momentum $(L)$ components, which are in turn expanded in coupled partial waves. Since the various $L$-components must be combined coherently to produce the ionization amplitude, it is essential 

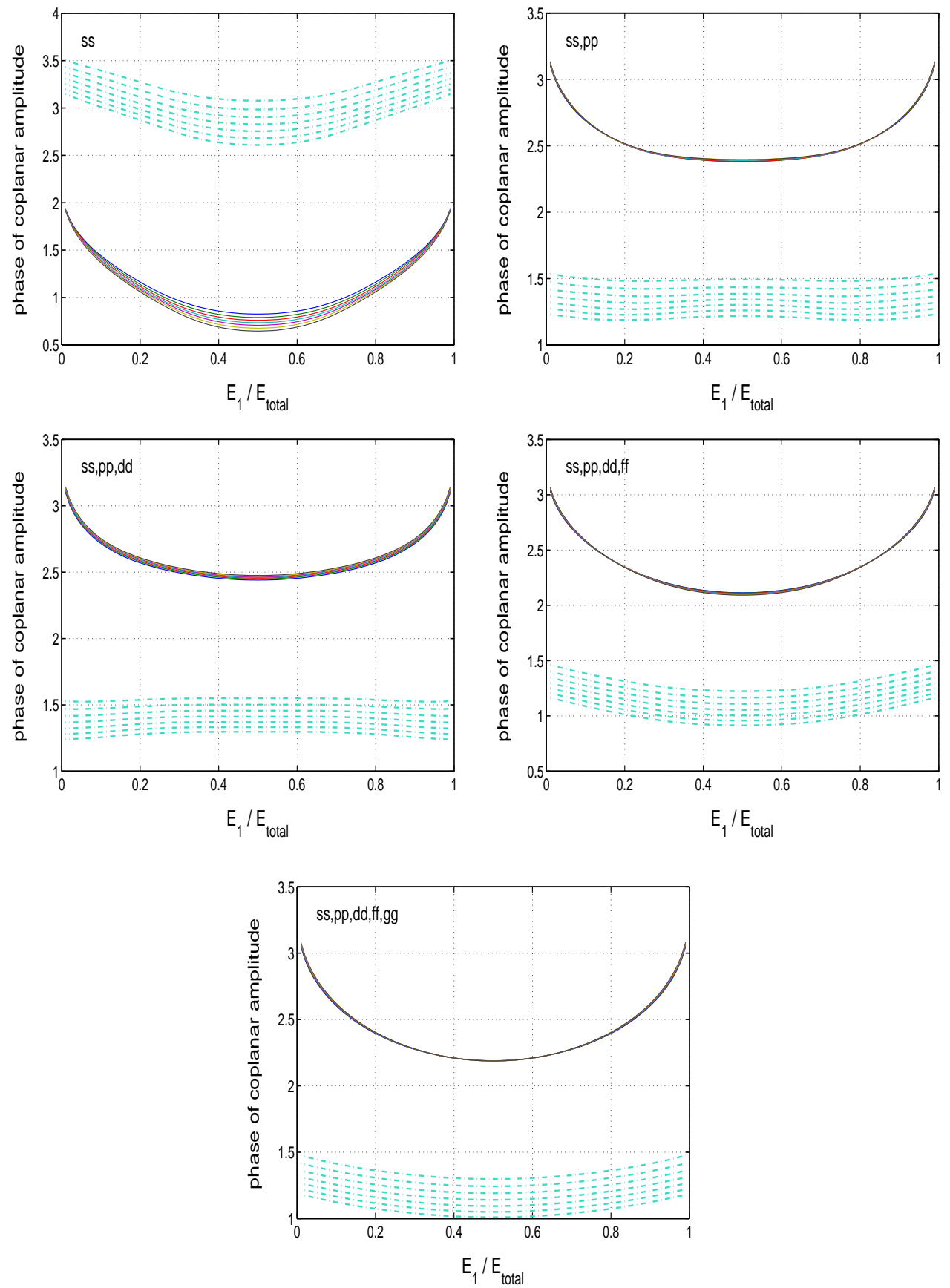

Figure 7. Phase of the $L=0, S=0$ component of the e- $H$ ionization amplitude as a function of the number of coupled partial-wave channels included. The incident energy is $30 \mathrm{eV}$ and the angle between the ejected electrons is 120 degrees. The dashed curves are the raw phases from the amplitudes computed using Eq.(133) for a series of hyperradius values between 102 and $150 \mathrm{bohr}$. The solid curves are obtained by removing the volume dependent Peterkop phase. 
to maintain phase coherence between the various components if one is to compute accurate differential cross sections.

The derivation of the Peterkop phase for the full problem is a straightforward generalization of the line of reasoning used above [3]. For the choice $z_{1}=z_{2}=1$, the Peterkop phase for the full problem,

$$
-1 /\left|\mathbf{k}_{1}-\mathbf{k}_{2}\right| \ln (2 K \rho),
$$

depends on the speed and direction of ejection of both electrons. Each total angular momentum component of the amplitude should carry the same Peterkop phase, provided the calculation is converged in partial waves and provided the same value of hyperradius is employed for each component. When the Peterkop phase is correctly constructed, it factors out of the total amplitude and makes no contribution to any observable cross section [68].

Employing the same wave functions used to compute the triple differential cross sections we previously reported [70], we computed the phases of the ionization amplitudes and applied the Peterkop phase correction. Figure 7 displays results for the $L=0, S=0$ component of the amplitude at a total energy of $30 \mathrm{eV}$ for the case where the angle between the ejected electrons is 120 degrees. The various panels show the raw and corrected phases for seven different values of $\rho$ as more partial waves are included in the calculation. The convergence of the corrected phase toward a $\rho$ independent result provides a sensitive test of the overall quality of the calculation. These tests also provide a stunning demonstration that the computed wave functions do indeed display properties demanded by the formal theory.

\section{Accurate amplitudes for electron impact ionization of the hydrogen atom}

Calculating the scattered wave is by far the most computationally intensive step in this approach. The partial wave expansion of the scattered wave for the e-H problem, given in Eq.(105), when substituted into the driven Schrödinger equation (Eq.(102)), gives a set of coupled two-dimensional equations of the form:

$$
\begin{aligned}
{\left[E+\frac{1}{2} \frac{\partial^{2}}{\partial r_{1}{ }^{2}}+\frac{1}{2} \frac{\partial^{2}}{\partial r_{2}{ }^{2}}+\frac{1}{r_{1}}+\frac{1}{r_{2}}\right] \psi_{l_{1} l_{2}}^{L}\left(r_{1}, r_{2}\right) } & -\sum_{l_{1}^{\prime} l_{2}^{\prime}}\left\langle l_{1} l_{2}|| l_{1}^{\prime} l_{2}^{\prime}\right\rangle \psi_{l_{1}^{\prime} l_{2}^{\prime}}^{L}\left(r_{1}, r_{2}\right) \\
& =\chi_{l_{1} l_{2}}^{L}\left(r_{1}, r_{2}\right)
\end{aligned}
$$

Formulas for the terms $\left\langle l_{1} l_{2}|| l_{1}^{\prime} l_{2}^{\prime}\right\rangle$ and $\chi_{l_{1} l_{2}}^{L}$, which come from the partial wave expanssions of $1 /\left|\mathbf{r}_{1}-\mathbf{r}_{2}\right|$ and the RHS of Eq.(102), respectively, are given in ref.[52]. In the ECS approach, these coupled equations are solved on a complex-scaled grid using one of the methods described in section 3 and the solutions are then used in constructing the cross sections of interest.

\subsection{Numerical methods}

We have calculated and reported results for e- $\mathrm{H}$ ionization using the ECS method at collision energies from $1 \mathrm{eV}$ above threshold up to $54.4 \mathrm{eV}$ [51, 52, 69, 70, 77, 78, 79, 80]. For collision energies ranging from a few $\mathrm{eV}$ above threshold up to $25 \mathrm{eV}$, we typically use grids whose real parts extend to $\sim 130$ bohr and include contributions from total angular momentum values up to $L=9$. In this energy range, as many as thirty 
coupled partial wave channels, depending on the $L$-value considered, are required for convergence. As the energy increases, more angular components are required, but one can use smaller grids. At $54.4 \mathrm{eV}$, which is the highest energy we considered, our $a b$ initio calculations included angular momentum components up to $L=13$ and grids whose real parts extended to $80 \mathrm{bohr}$. In practice, it is very difficult to obtained converged cross sections above $50 \mathrm{eV}$ with our methods because of the large number of contributing partial waves, but fortunately it has been found [78] that $50 \mathrm{eV}$ is just about the energy at which perturbative methods become applicable.

All of our published results on the full e- $\mathrm{H}$ problem were calculated using highorder finite difference. For the largest grids, we typically use $\sim 500$ points in each radial dimension, which requires us to solve linear systems of a few million complex linear equations. The finite element-DVR approach, which is more efficient than finite difference, would allow us to extend the grids to $\sim 200$ bohr with roughly the same number of terms, but we have yet to apply this method to the full $\mathrm{e}-\mathrm{H}$ problem.

It is impractical to solve linear systems of this size directly, so we must employ iterative methods The eigenvalue spectrum of the complex exterior-scaled Hamiltonian is such that no known iterative algorithm will converge to solution without preconditioning. Therefore, finding a suitable pre-conditioner for the coupled equations was a necessity. We found that the set of uncoupled radial equations, obtained by setting $\left\langle l_{1} l_{2}|| l_{1}^{\prime} l_{2}^{\prime}\right\rangle=0$ for all $\left(l_{1}^{\prime}, l_{2}^{\prime}\right) \neq\left(l_{1}, l_{2}\right)$, have numerical properties similar to the coupled equations, but require solving linear systems only as big as the total number of radial grid points $(\sim 250,000)$. The solutions of the uncoupled equations were found to be a suitable preconditioner for the coupled equations, which in turn were solved iteratively using a congugate gradient squared algorithm. Details can be found in ref.[52].

While the first ECS results for e-H ionization were obtained by extrapolating the quantum mechanical flux [51,52], the most accurate results obtained to date have used the underlying integral amplitudes, as outlined above, and those are the results which we will summarize here.

\subsection{Single and double differential cross sections}

The single differential cross section measures the energy sharing between the ejected electrons, irrespective of their angles of ejection. This quantity, which reduces to an incoherent sum of partial-wave contributions, is plotted in Fig. 8 for a range of collision energies. We show the total SDCS along with the individual singlet and triplet contributions. At energies above $25 \mathrm{eV}$, the cross sections become increasingly bowshaped, reflecting the fact that at high energies the tendency is for the fast incident electron to retain much of its energy and to produce a slow secondary electron. As the collision energy decreases, the SDCS begins to flatten. At $17.6 \mathrm{eV}$ and below, the SDCS is essentially flat, in agreement with the predictions of Wannier theory [81], and begins to reveal subtle undulations, also in accord with the predictions of semiclassical theory [82].

The individual contributions to the $25 \mathrm{eV}$ incident energy SDCS for different values of $L$ and $S$ are shown in Fig. 9. Each of these components is symmetric about $E / 2$, reflecting the indistinguishability of the electrons. Note that for both the singlet and triplet cases, $L=0$ does not give the dominant contribution to the cross section.

Further insight about the convergence of the SDCS at low energy is gained by looking at the convergence of a single $L$ component as the number of partial waves 

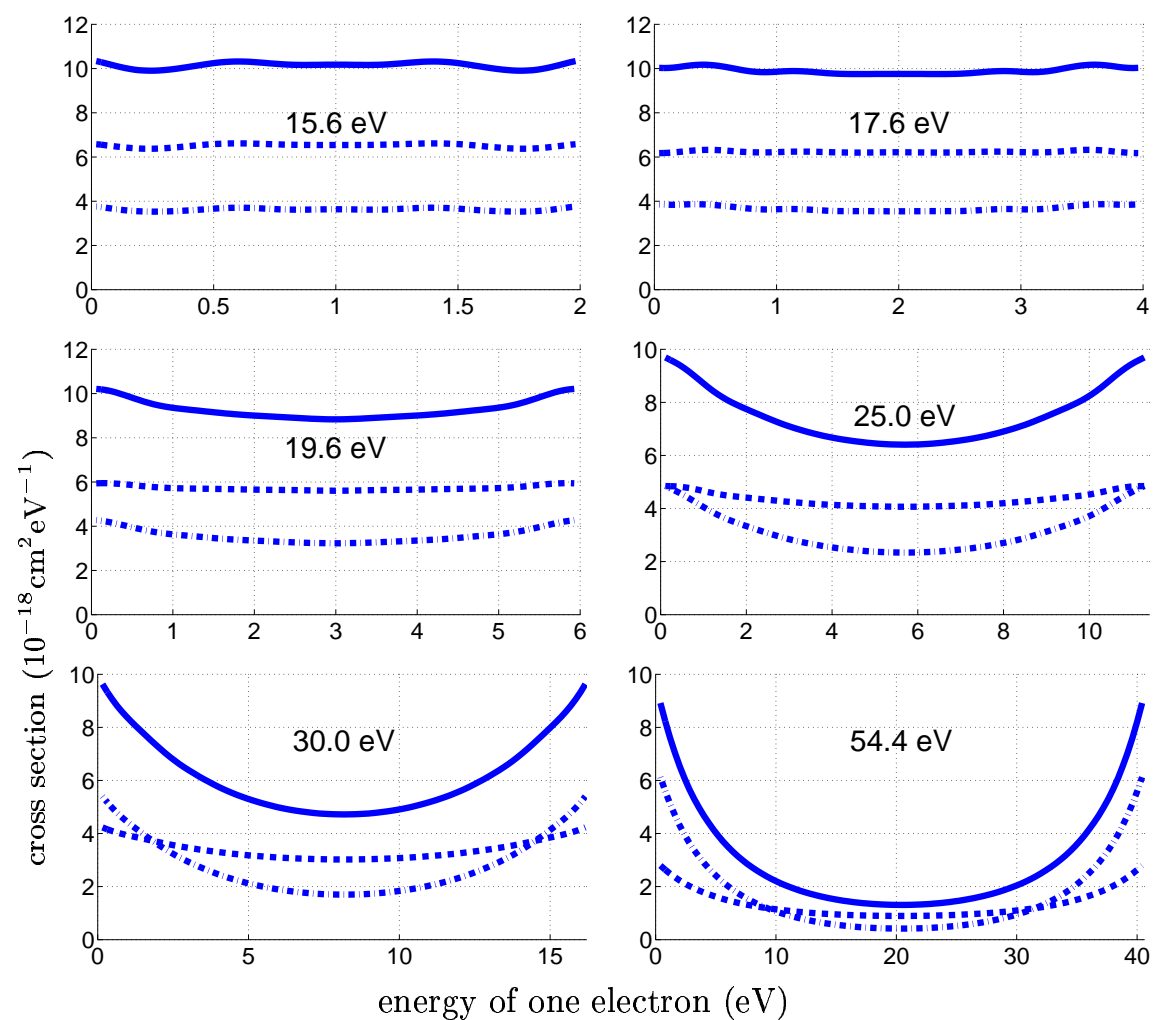

Figure 8. Single differential cross sections for e-H scattering at the collision energies indicated. Individual components for singlet (dashed line) and triplet (dot-dash line) are shown.
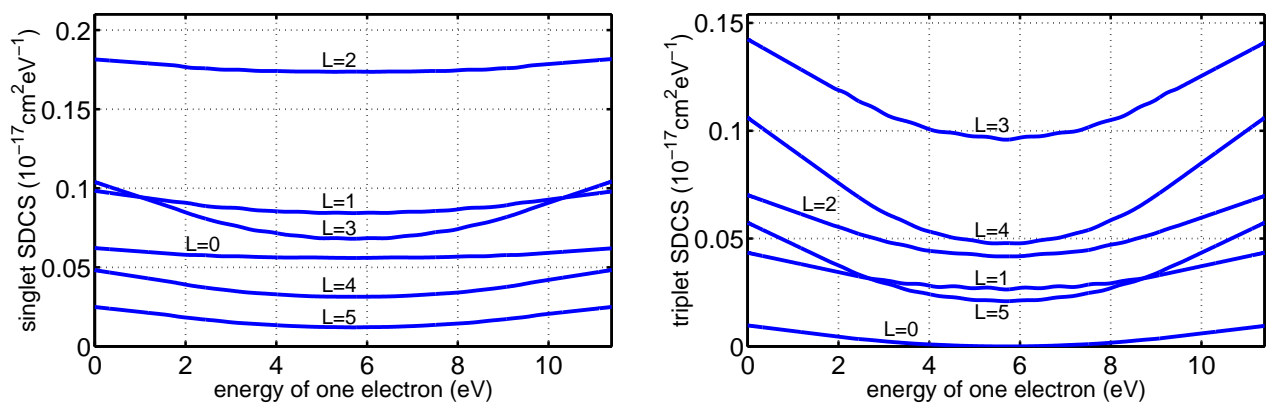

Figure 9. Components of the SDCS (with spin factors included) for electronhydrogen scattering at $25 \mathrm{eV}$ incident energy corresponding to particular total spin and angular momentum quantum numbers. 

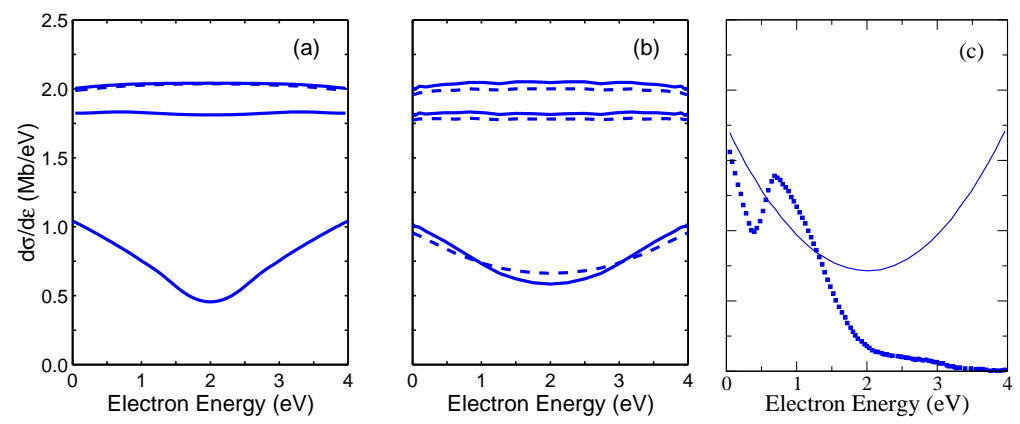

Figure 10. Single differential cross sections for electron-impact ionization of hydrogen at $4 \mathrm{eV}$ above threshold, for $L=0, S=0$. Statistical weights are not included. The lowest curve is the result with only the $s s$ partial wave in the calculations and the middle curve couples $s s+p p$. The upper set includes up to $d d$ (solid) and $f f$ (dashed). case using $s s+p p+d d$ pairs. The results in the left panel were computed using ECS, while those on the right were obtained from a time-dependendent close-coupling calculation [69]. $\left(1.0 \mathrm{Mb}=1.0 \times 10^{-18} \mathrm{~cm}^{2}\right)$.

is increased. Figure 10 shows results for the $L=S=0$ component of the SDCS at $17.6 \mathrm{eV}$. When only s-waves are included, which is the much studied TemkinPoet model, the cross section exhibits a pronounced V-shape. Adding the $p p$ terms in the expansion increases the magnitude of the cross section substantially, showing the critical importance of the dipole coupling in the $L=0$ cross section. The most striking effect of coupling higher angular momentum terms to the ss pair is to flatten the overall differential cross section, an effect, as we have mentioned above, that is consistent with semiclassical and Wannier theory.

The SDCS is not a quantity for which direct measurements exist. The SDCS is generally obtained by integrating the double differential cross section (DDCS) over the angle of the observed electron. There have been two experimental determinations of the e-H DDCS. Shyn [83] reported measurements of the DDCS in 1992 for incident electron energies between 25 and $250 \mathrm{eV}$. The very recent absolute cross section measurements of Childers et al. [80] cover the energy range from just $1 \mathrm{eV}$ above threshold to $40 \mathrm{eV}$ and include measurements of the DDCS for ejected electrons both above and below $E / 2$. Figure 11 shows a representative sampling of DDCS results at an incident electron energy of $25 \mathrm{eV}$. There is evidently excellent agreement between experiment and both the theoretical ECS and CCC results over the entire range of energy sharing. Similar agreement is found at other collision energies. Note that there is no symmetry in the DDCS about $E / 2$, while the integral under the various curves must yield a symmetric SDCS.

\subsection{Triple differential cross sections}

The triple differential cross section (TDCS) offers the most stringent test for judging the fundamental correctness of any method that proposes to properly treat the threebody Coulomb problem from first principles. Unlike the SDCS, the TDCS requires a construction of the complete ionization amplitude so that any phase inconsistencies among the partial wave amplitudes $\left(f_{l_{1}, l_{2}}^{L}\right.$ in Eq.(109)) would adversely affect the calculated TDCS. In other words, the SDCS tests only the magnitudes of the calculated 

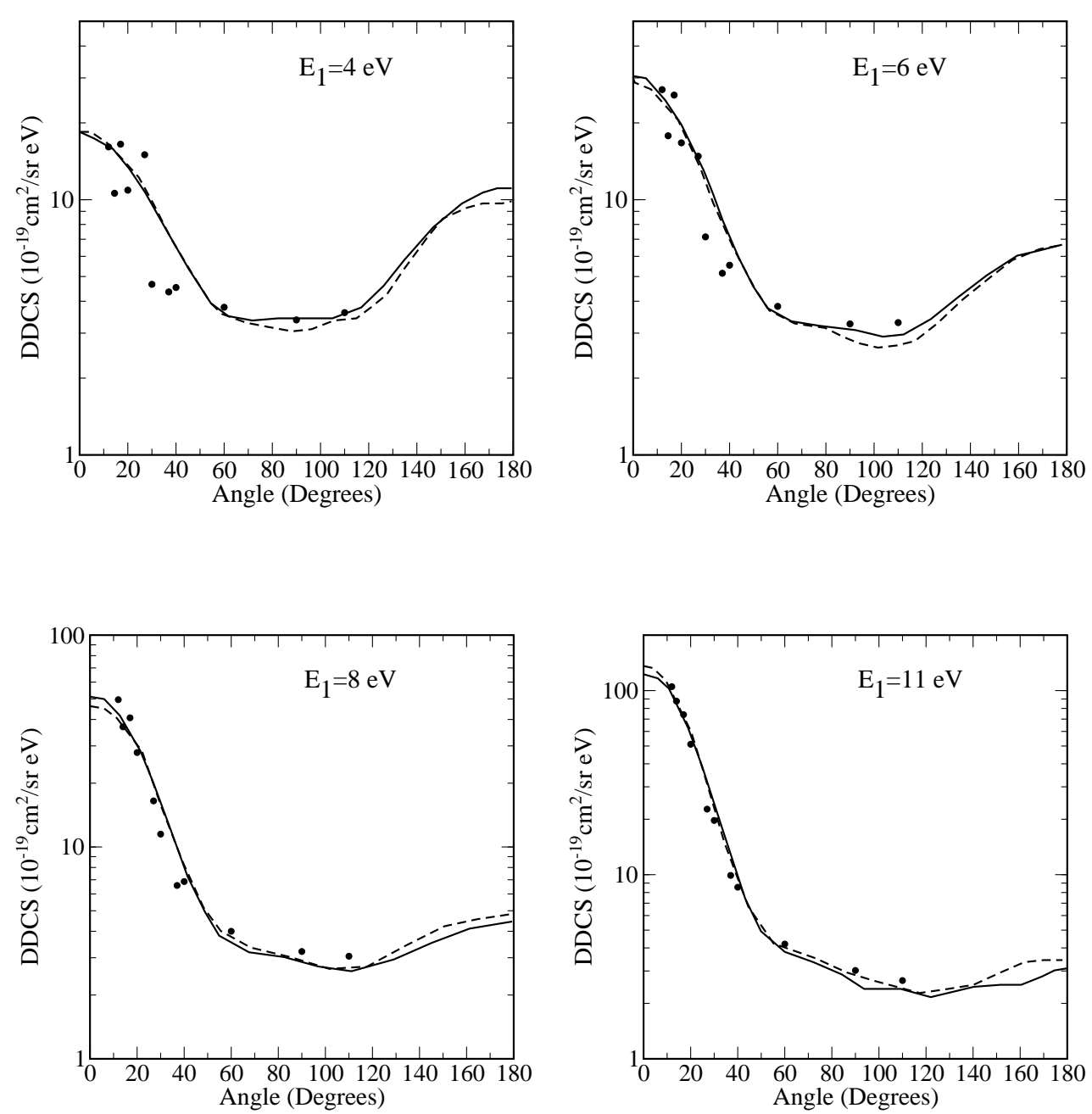

Figure 11. e- $\mathrm{H}$ double differential cross sections at $25 \mathrm{eV}$ incident electron energy from ref. [80]. Ejected electron values of 4, 6, 8 and $11 \mathrm{eV}$. Solid curves: ECS. Dashed curves: CCC. Circles: experiment.

amplitudes but the TDCS also tests the phases.

In 1996 Röder et al. [84] published a set of TDCS for e-H ionization at collision energies between 15.6 and $54.4 \mathrm{eV}$. All of this data was taken in the so-called "symmetric co-planar geometry" where the incident and ejected electrons all lie in the same plane and the two ejected electrons have the same energy. The data was not absolute, but was internormalized at each energy. In 1997 Röder et al. [85] provided a small set of absolute data at 15.6 and $17.6 \mathrm{eV}$, which could then be used to put the earlier data at these two energies on an absolute scale. Much has been written about this "absolute" data over the past few years. Inconsistencies in the original $17.6 \mathrm{eV}$ data were pointed out by Bray [86]. The data was subsequently remeasured [87] and reanalyzed [79] and shown to agree well with the predictions of both ECS and CCC calculations. 

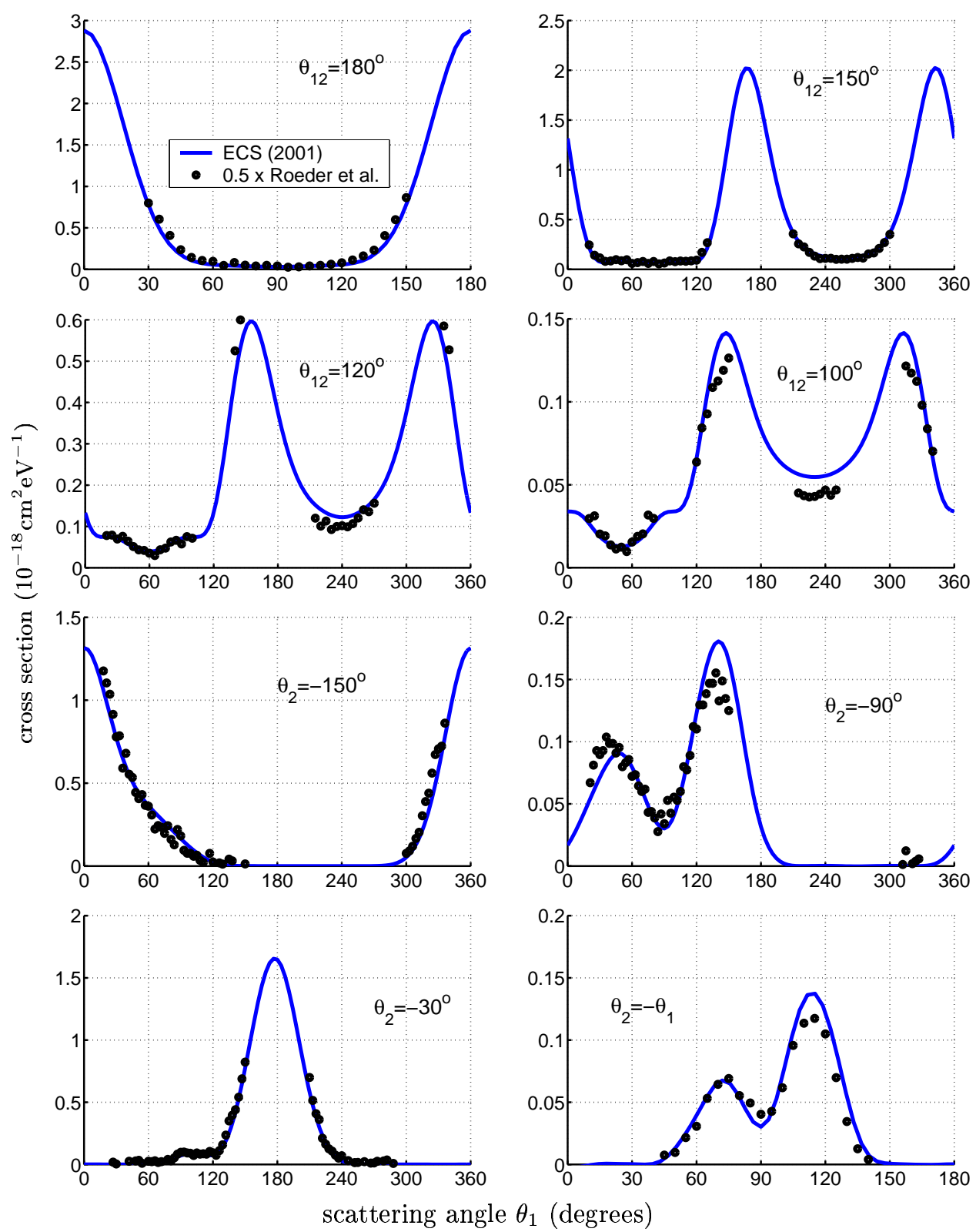

Figure 12. Equal-energy sharing TDCS at $15.6 \mathrm{eV}$ incident energy for various coplanar geometries. Absolute experimental data $[84,85]$ has been multiplied by 0.5 . 

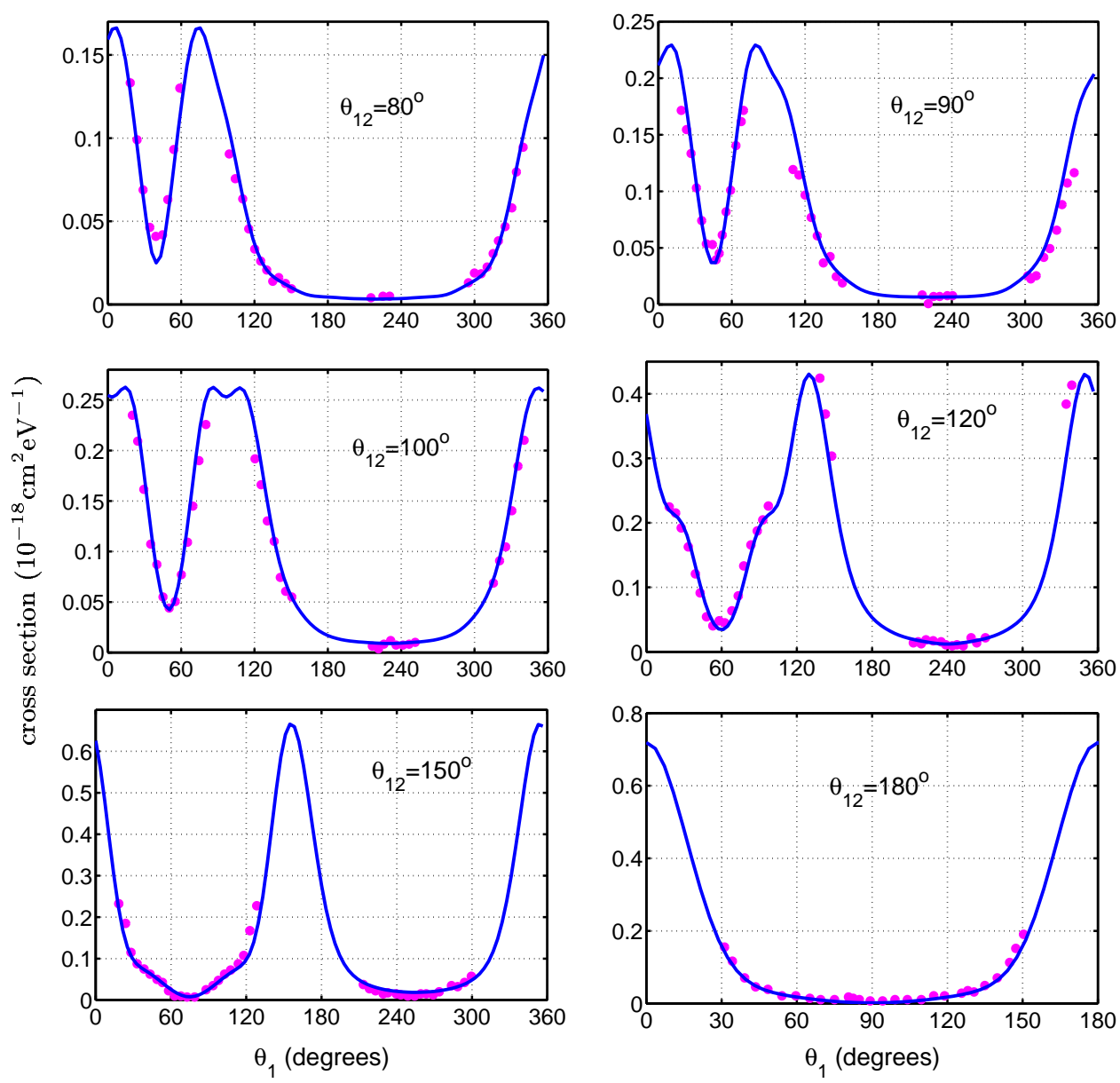

scattering angle $\theta_{1}$ (degrees)

Figure 13. Equal-energy sharing, coplanar TDCS for $25 \mathrm{eV}$ incident energy. Internormalized measurements [84], originally reported in arbitrary units, were multiplied by 0.16 to fit calculated cross section. Solid curves: ECS results [70].

The 15.6 eV TDCS data does not suffer from any internal inconsistencies. However, there is strong evidence that the absolute normalization is incorrect [86]. Figure 12 compares the results of ECS calculations with the experimental data at 15.6 $\mathrm{eV}$. It is clear that reducing the experimental cross sections by a factor of 2 brings them into excellect agreement with theory, both in shape and in magnitude. Other calculations [85, 88] also support this conclusion.

Figure 13 shows TDCS data for $25 \mathrm{eV}$ incident energy. The experimental results for this energy were reported in arbitrary units, but since they are internormalized, we have chosen a single scaling factor for plotting the data. Once again, the agreement between the scaled data and the ECS calculations is excellent. There is similarly good agreement between the ECS results and experiment at other energies as well.

The reduction of this problem to computation means that with accurate 

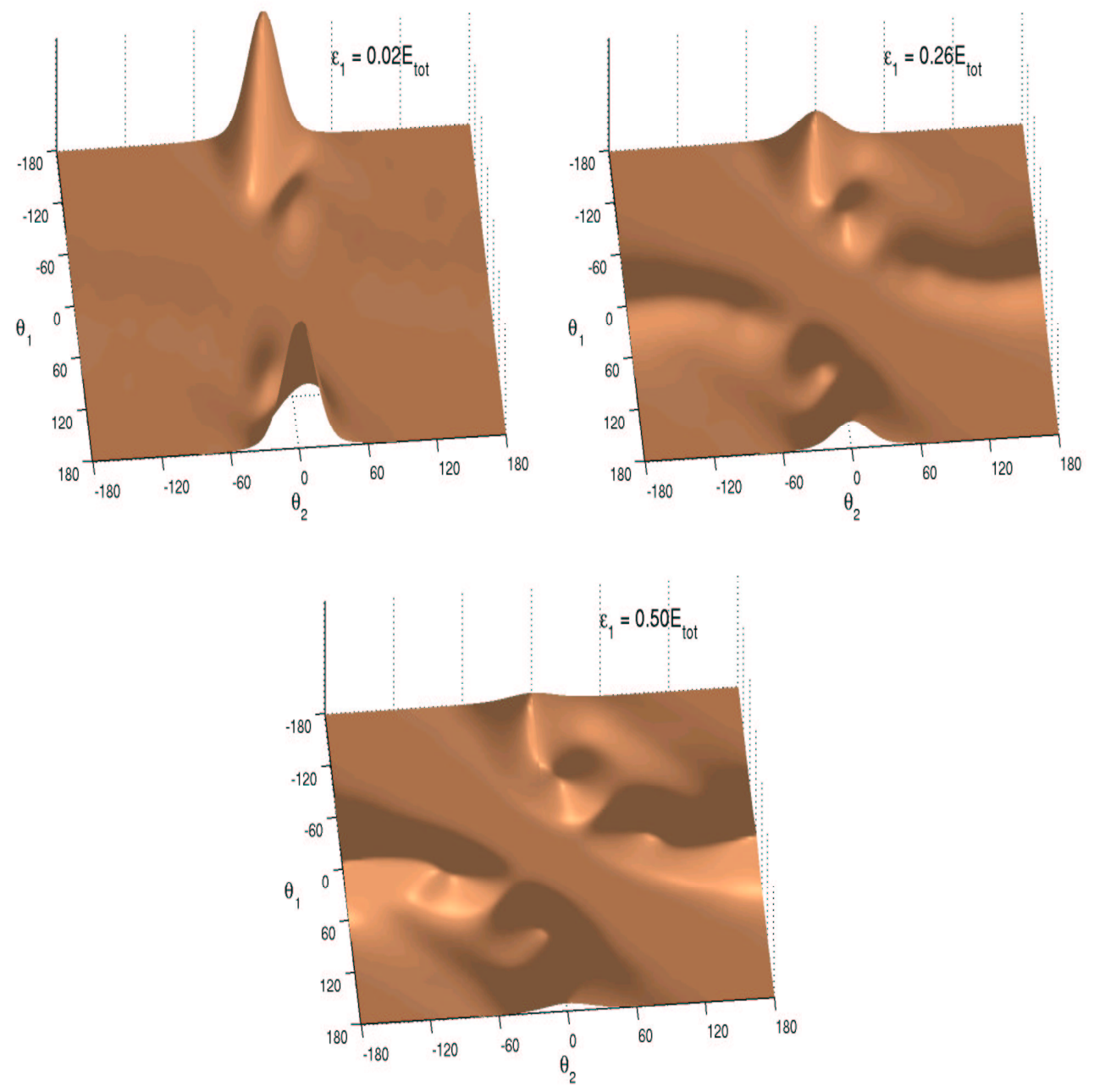

Figure 14. TDCS vs $-\theta_{1}$ and $\theta_{2}$ for different energy sharings at $25 \mathrm{eV}$ incident energy. Top left: $\epsilon_{1}=1 / 50 E$. Top right: $\epsilon_{1}=1 / 4 E$. Bottom: $\epsilon_{1}=1 / 2 E$.

calculations we can now confidently explore the dynamics at geometries that have never been measured. A glimpse of what is now possible is given in Fig. 14, which shows the TDCS at $25 \mathrm{eV}$ for three different energy sharings as a function of the angles of both ejected electrons. The TDCS for nonequal energy sharing demonstrates in detail how the angular distributions change as the fraction of the ejection energy taken by one electron varies from zero to one. For this simple system it will shortly become a routine matter to computationally explore noncoplanar geometries together with with unequal energy sharing only a few volts above threshold. At this stage it would be useful to have a final, absolute, experimental benchmark for one or two such configurations. 


\section{Double photoionization of the helium atom}

Our recent treatment of double photoionization of the ground state of helium [58] is an example of the use of B-splines in a two-electron exterior scaling calculation. In this review we limit ourselves to only a brief description of the calculation and a summary of the comparison of the results with experiment, concentrating on some of the salient aspects of the physics.

The double photoionization of an atom by a single photon is a process that is exquisitely sensitive to the correlation effects of the initial state, which we denoted as $\left|\Gamma_{0}\right\rangle$ in Eq.(98). If we consider the simplest model for this process in the helium atom, we would represent the initial state as a single-determinant Hartree-Fock wave function with the configuration $\left|1 s^{2}\right|$. With only one photon, the double ionization transition, $\left|1 s^{2}\right| \rightarrow\left|k s, k^{\prime} p\right|$, would have zero probability if the continuum orbitals $k s$ and $k^{\prime} p$ were taken to be Hartree-Fock virtual orbitals. Although such an approximation would be an extreme over simplification of the physics, it is nonetheless why it is sometimes said that single-photon double ionization is a "pure correlation" process.

\subsection{Numerical solution of the first-order wave equation}

The first requirement, because of the sensitivity of this double ionization process to correlation effects in the initial state, is an accurate configuration interaction (CI) description of the ground state. For the initial state we used configurations containing orbital angular momenta up to $l=4$ made up of Slater type orbitals with exponents of $2.4,3.6,4.8,6.0$ and 6.8 for $l=0, \ldots, 4$ respectively, to give a total of 115 configurations. These Slater orbitals are expanded in the B-spline basis described below, so that the same underlying basis of B-splines is used for both the initial and final states. The ground state energy given by this calculation is -2.903198 hartree compared to the exact value [89] of -2.903724 hartree and is thus close to the $l=4$ limit for the energy of the initial state.

Calculations for double ionization can generally be performed using smaller values of $R_{0}$, and therefore smaller basis sets, than would be required for the isoelectronic electron-impact ionization process (electron-impaction ionization of hydrogen in this case). The reason is that the Coulomb attraction of the nucleus dominates even at relatively short distances over electron-electron repulsion in the final state except at energies near threshold. For double ionization of the helium ground state, a value of $R_{0}=35 \mathrm{a}_{0}$ suffices for photon energies $20 \mathrm{eV}$ above the double ionization threshold and higher.

A small basis set suffices as well, and using 53 B-splines for each one-electron atomic symmetry is adequate for this calculation. Those B-splines were defined on a computational grid with $47 \mathrm{~B}$-spline knot points over the first $42.0 \mathrm{a}_{0}$, and 6 additional knot points on the remaining complex contour out to $R_{\max }=80 \mathrm{a}_{0}$. Typically in ECS calculations a less dense grid can be used on the complex part of the countour, where the wave function is decaying exponentially. The angular momentum expansion of $\Psi_{1}^{+}$in Eq.(116) included $l$ values up to $l_{\max }=4$, giving us contributions from the $k s k p, k p k d, k d k f$, and $k f k g$ double continua. Using these 53 B-splines and 4 double continua, we have a total of 11,236 configurations in the CI representation of $\Psi_{1}^{+}$. All the results we present below were computed in the velocity gauge, although the results in the length gauge are essentially the same [58]. 


\subsection{Single and triple differential cross sections}

A initial sense of how the double photoionization cross sections reflect the electron correlation in the initial state can be had by examining the contributions to the single differential cross section, defined in Eq.(112), for various partial waves. For example, the contribution of the double continuum corresponding to $k d k f$ arises only from the correlating contributions to the ${ }^{1} S$ ground state of the form $\left|d d^{\prime}\right|$ and $\left|f f^{\prime}\right|$, i.e., configurations with two orbitals of those angular momenta. The various contributions to the SDCS at a photon energy $20 \mathrm{eV}$ above threshold are shown in Fig. 15. The contributions of the double continua $k s k p$ and $k p k d$ are of similar magnitude $\left(\left|p p^{\prime}\right|\right.$ contributes to both), but the contributions of double continua with higher partial waves are smaller reflecting the ever smaller contributions to angular correlation in the initial state of higher angular momenta.

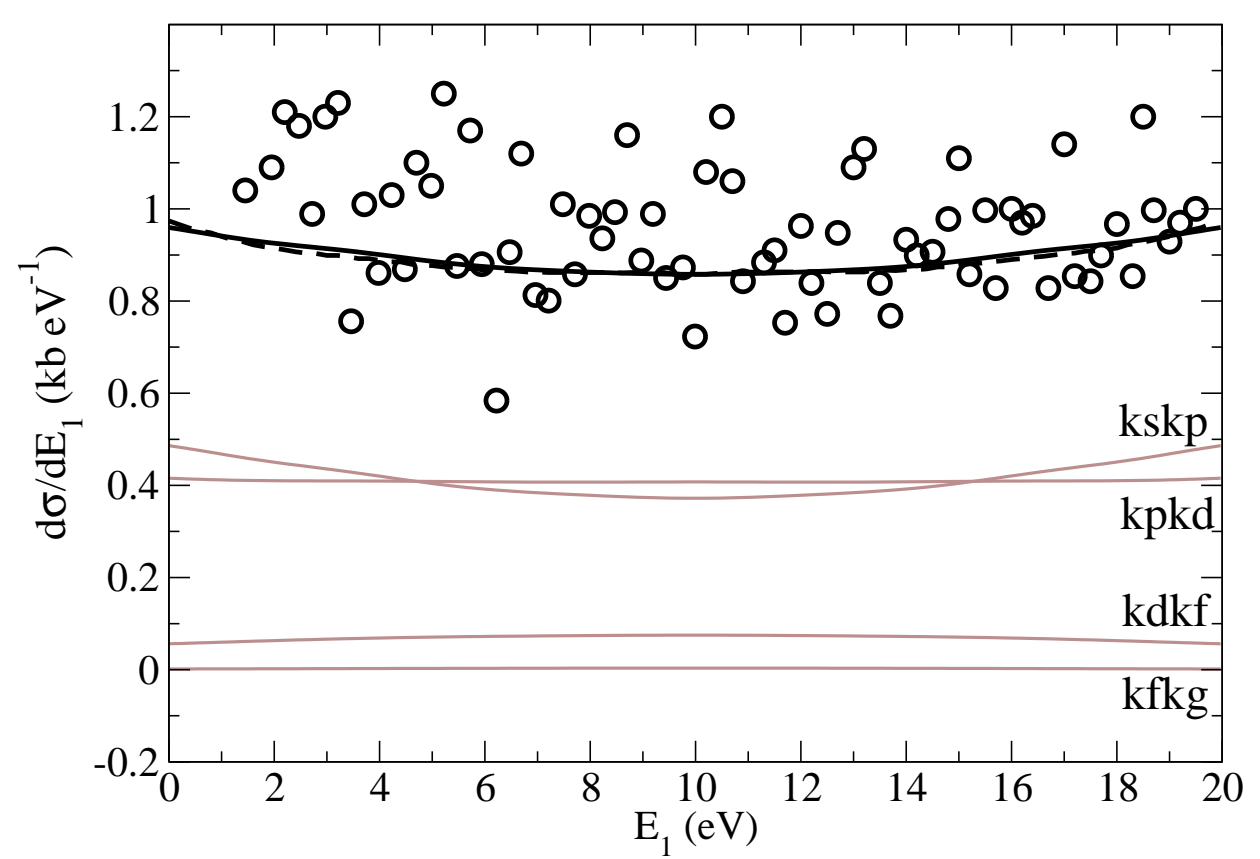

Figure 15. SDCS for photon energy $20 \mathrm{eV}$ above threshold. Circles: experiment by Wehlitz et al. [90]. Dashed curve: TD-CC calculations by Colgan et al. [91]. Thick solid curve: ECS [58]. Lighter solid curves: contributions to SDCS from each noted double continuum.

Triple differential cross sections reflect those correlation effects as well as specific optical selection rules. The left column of Fig. 16 shows some examples, again for a photon energy $20 \mathrm{eV}$ above threshold. In the left column of that figure the direction of one electron is always along the polarization diretion, $\theta_{1}=0^{0}$, and we see in the top panel the selection rule that prevents electrons of the same energy from going out in opposite directions $\left(\theta_{2}=180^{\circ}\right.$ in this case). However the other panels in that column show how for unequal energy sharing no such selection rule exists. The zero (or very small) value of the cross section at $\theta_{2}=0^{0}$ reflects the effects of electron repulsion preventing the two electrons from exiting in the same direction, and in contrast to the selection rules the contribution from the double continua with higher partial waves 
is required to resolve that effect computationally. Also shown in Fig. 16 are the results of the TDCC and HRM-SOW methods, both of which also describe these triple differential cross sections well.
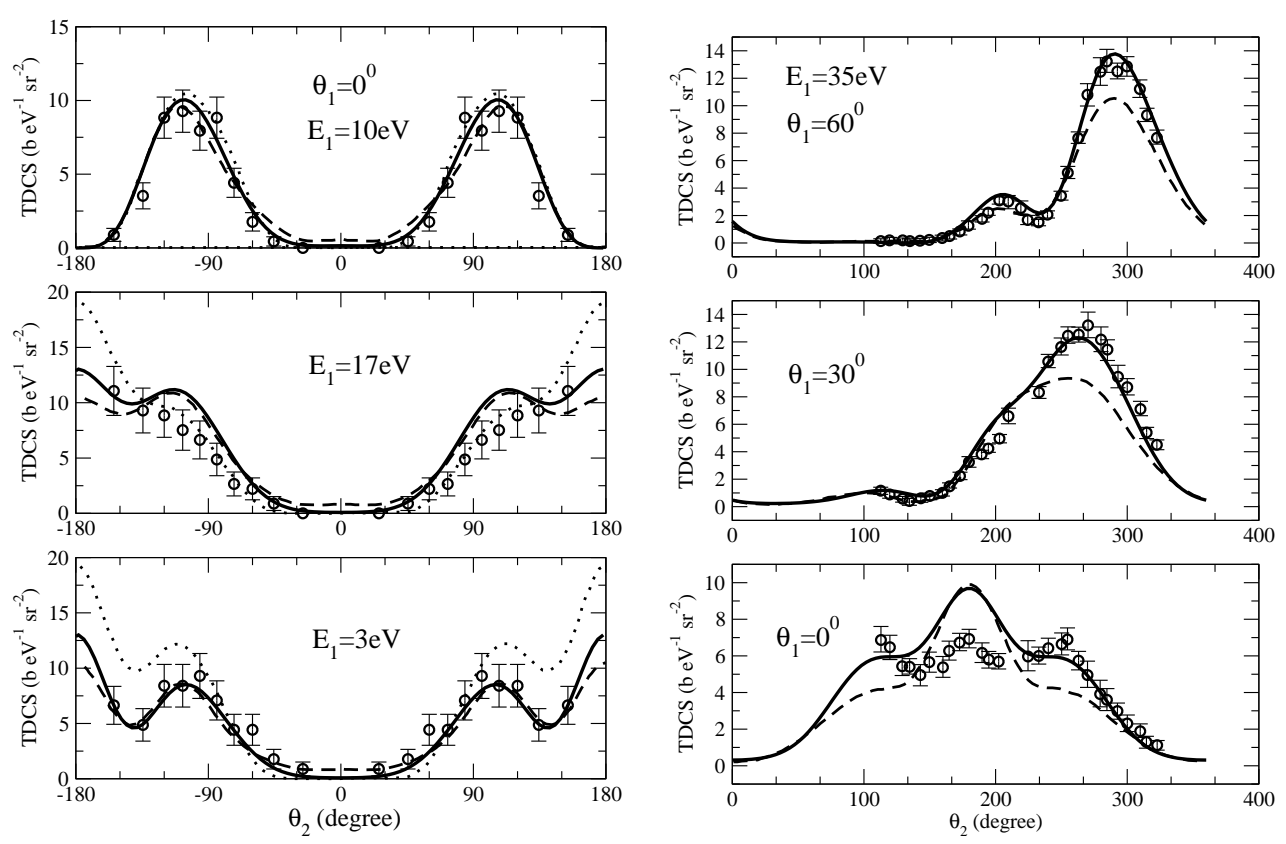

Figure 16. Left column: TDCS for photon energy $20 \mathrm{eV}$ above threshold, at various energy sharings for $\theta_{1}=0^{0}$. Circles: experiment by Braüning et al. [92]. Dashed curve: TD-CC calculations by Colgan et al. [91]. Dotted curve: HRM-SOW calculations by Selles et al. [93]. Thick solid curve: ECS [58]. Right column: TDCS for photon energy $40 \mathrm{eV}$ above threshold, in the unequal energy sharing of $E_{1}=35 \mathrm{eV}$ and $E_{2}=5 \mathrm{eV}$ at various values of $\theta_{1}$ of the $35 \mathrm{eV}$ electron. Circles and dashed curve: experiment and CCC calculation of Ref. [94]. Thick solid curve: ECS [58]

The triple differential cross sections at unequal energy sharing for relatively low energies above threshold remain the most difficult to calculate and there occasionally remain small, but significant differences between various theoretical calculations. In the right column of Fig. 16 we compare with the experimental results of Bolognesi et al. [94] and the results of CCC calculations included in the same reference for an energy sharing $E_{1}=5$ and $E_{2}=35 \mathrm{eV}$, and $\theta_{1}$ varying from $0^{0}$ to $60^{\circ}$. We have normalized the relative experimental cross sections to our computed TDCS at $\theta_{1}=60^{\circ}$ and $\theta_{2}=30^{\circ}$ for this energy sharing, thereby fixing the normalization of the experiment. The results of CCC calculations from reference [94] are also shown in the right column of Fig. 16 with no scaling. Although both theoretical results generally reproduce the shapes of these these and similar TDCS plots [58], it can be seen that there remain significant differences between the theories and between the theories and the experiment. However, it cannot be concluded from these comparisons with experiment that the theoretical results from either of these high-quality calculations is definitively more accurate than the other.

A more complete comparison with experiment at $20 \mathrm{eV}$ involving various energy sharings is shown in Fig.17, where the comparison with experiment shows the excellent 
agreement of theory with absolute experimental measurements that is now the state of the art [58]. Two-dimensional plots of the TDCS versus the angles of the ejected
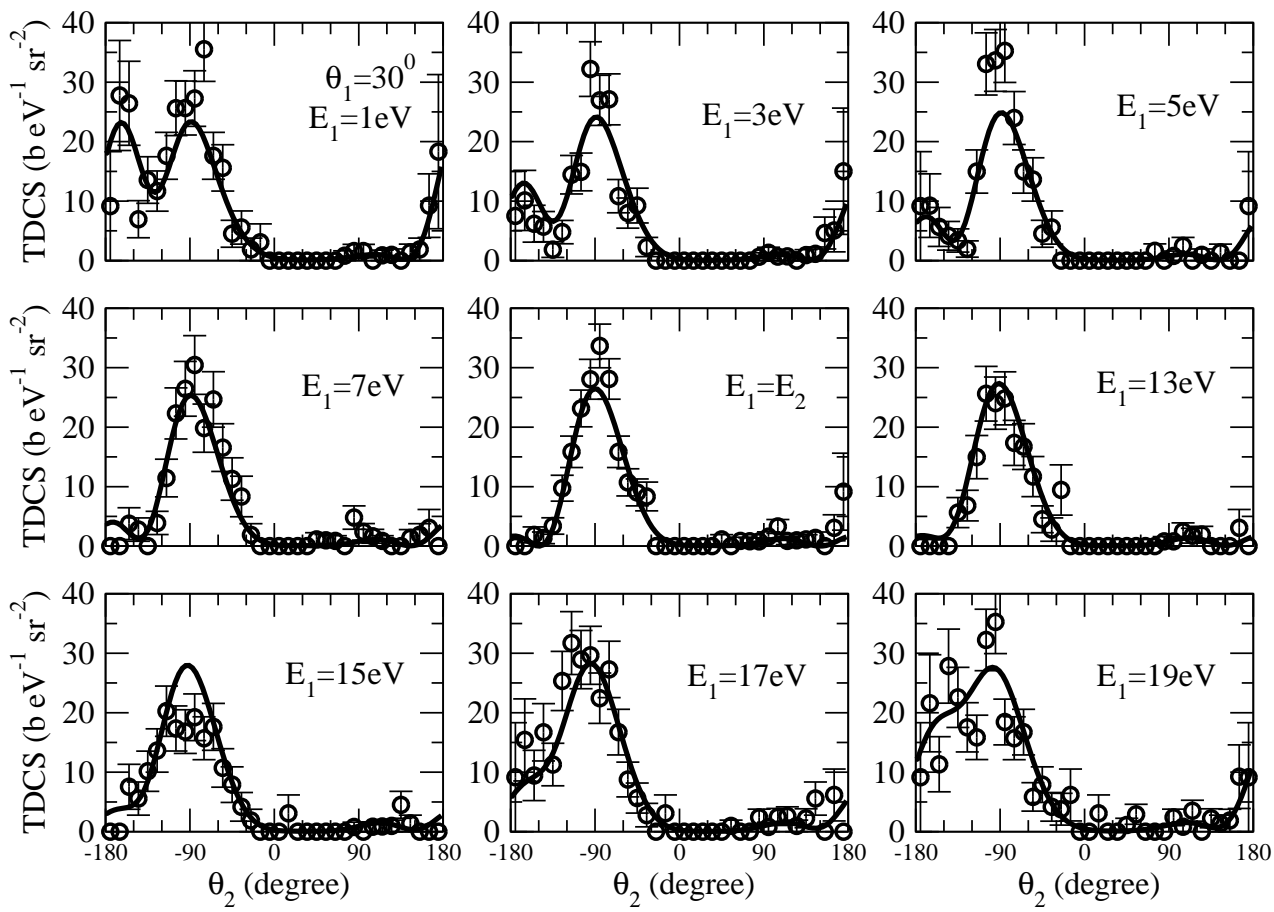

Figure 17. TDCS at $20 \mathrm{eV}$ above threshold for the geometry $\theta_{1}=30^{\circ}, \phi_{1}=\phi_{2}$. The panels show various energy sharings with the energy $E_{1}$ given in each panel. Circles: experiment by Braüning et al. [92]. Solid curve: ECS [58].

electrons like these are essential for conveying an accurate impression of the comparison between experiment and thoery, but it is difficult to glean a clear overall impression of the angular dependences of these cross sections from such graphs. In Fig.18 we plot a three-dimensional view of the TDCS for a photon energy $40 \mathrm{eV}$ above threshold and with the energy sharing $E_{1}=35 \mathrm{eV}$ and $E_{2}=5 \mathrm{eV}$. The dark arrow denotes the direction of ejection of the faster electron, while the surface is a plot of the corresponding TDCS as a function of the ejection angles of the slower electron. This plot immediately conveys the general impression of the dynamics of two electrons tending to exit the interaction region in opposite directions, due to the Coulombic repulsion between them, except when optical selection rules prevent them from doing so. The details of the changes in the cross section with varying $\theta_{1}$ (the direction of the faster electron) suggest the competition between those two effects.

Malegat et al. [95, 96] have derived a useful and compact representation of the TDCS for double photoionization by exploiting particular properties of the coupled spherical harmonics which were first noted by Kono and Hattori [97] and generalized by Malegat et al. in this application. For each energy sharing, the TDCS can be parametrized rigorously by two complex amplitudes, each of which is a function only 

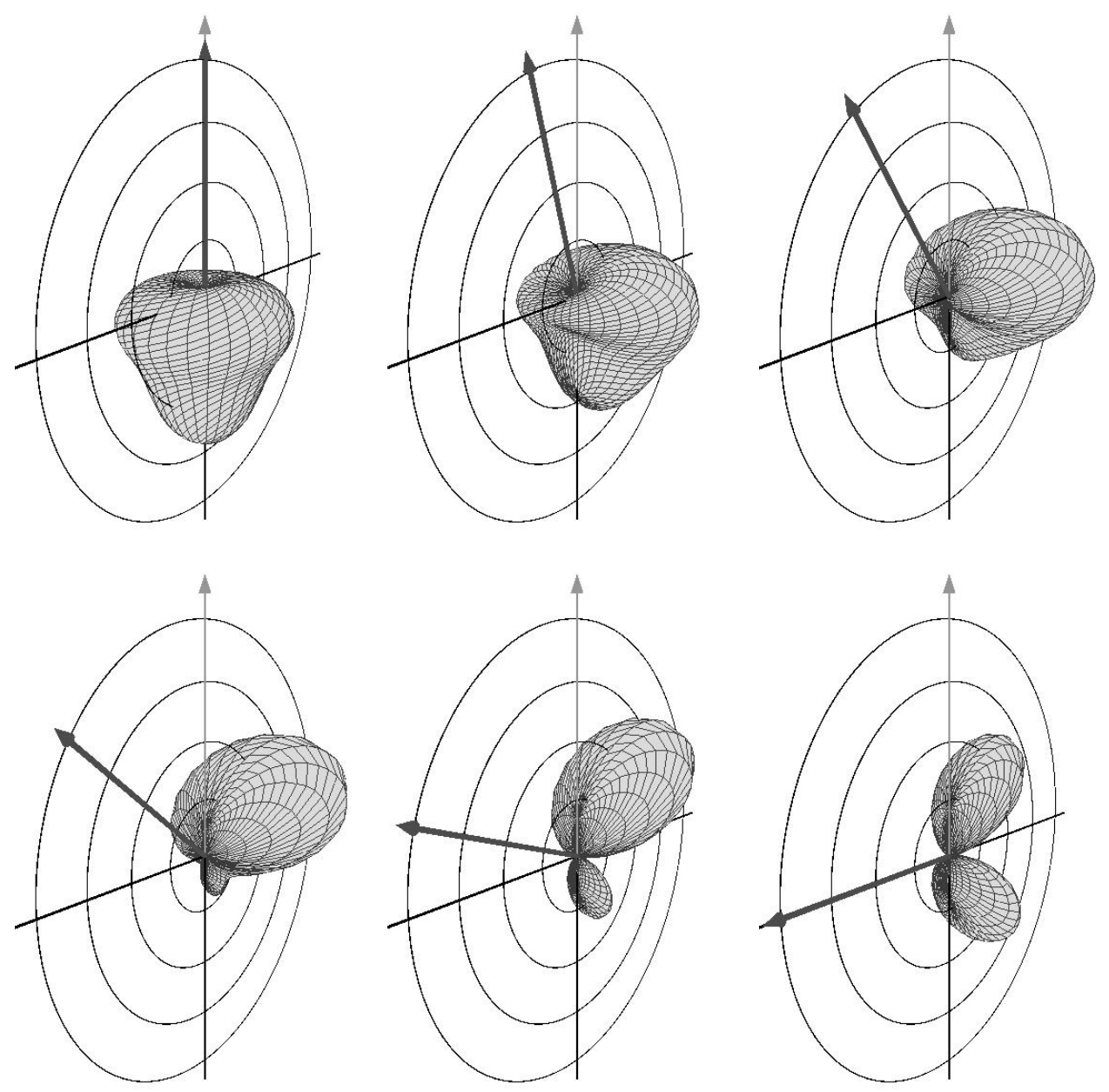

Figure 18. 3D representation of the TDCS for $40 \mathrm{eV}$ excess energy with energy sharing $E_{1}=35 \mathrm{eV}, E_{2}=5 \mathrm{eV}$. Light arrow (upwards on z-axis) is the polarization direction. The darker arrow is the direction of electron $1(35.0 \mathrm{eV})$, varying from $0^{0}$ to $90^{\circ}$ in steps of $18.0^{\circ}$.

of $\theta_{12}$, the angle between the two ejected electrons, and can be written in the form,

$$
\begin{array}{r}
\frac{d^{3} \sigma}{d \alpha d \Omega_{1} d \Omega_{2}}=\mid A_{g}\left(E_{1}, E_{2}, \theta_{12}\right)\left(\cos \theta_{1}+\cos \theta_{2}\right)+ \\
\left.A_{u}\left(E_{1}, E_{2}, \theta_{12}\right)\left(\cos \theta_{1}-\cos \theta_{2}\right)\right|^{2}
\end{array}
$$

The magnitude of each of the amplitudes, $A_{g}\left(E_{1}, E_{2}, \theta_{12}\right)$ and $A_{u}\left(E_{1}, E_{2}, \theta_{12}\right)$, and their relative phases can be determined by fitting experimental determinations of the cross sections, and they can be calculated directly by any of the theoretical methods that have been used successfully to treat the double photoionization process. A comparison of various calculations of these amplitudes for the case of the energy sharing $E_{1}=5 \mathrm{eV}$ and $E_{2}=35 \mathrm{eV}$ is shown in Fig. 19. Although some disagreements between the theoretical methods represented in that figure are noticeable, primarily for geometries at which the ungerade amplitude, $A_{u}$, is very small, current experiments 

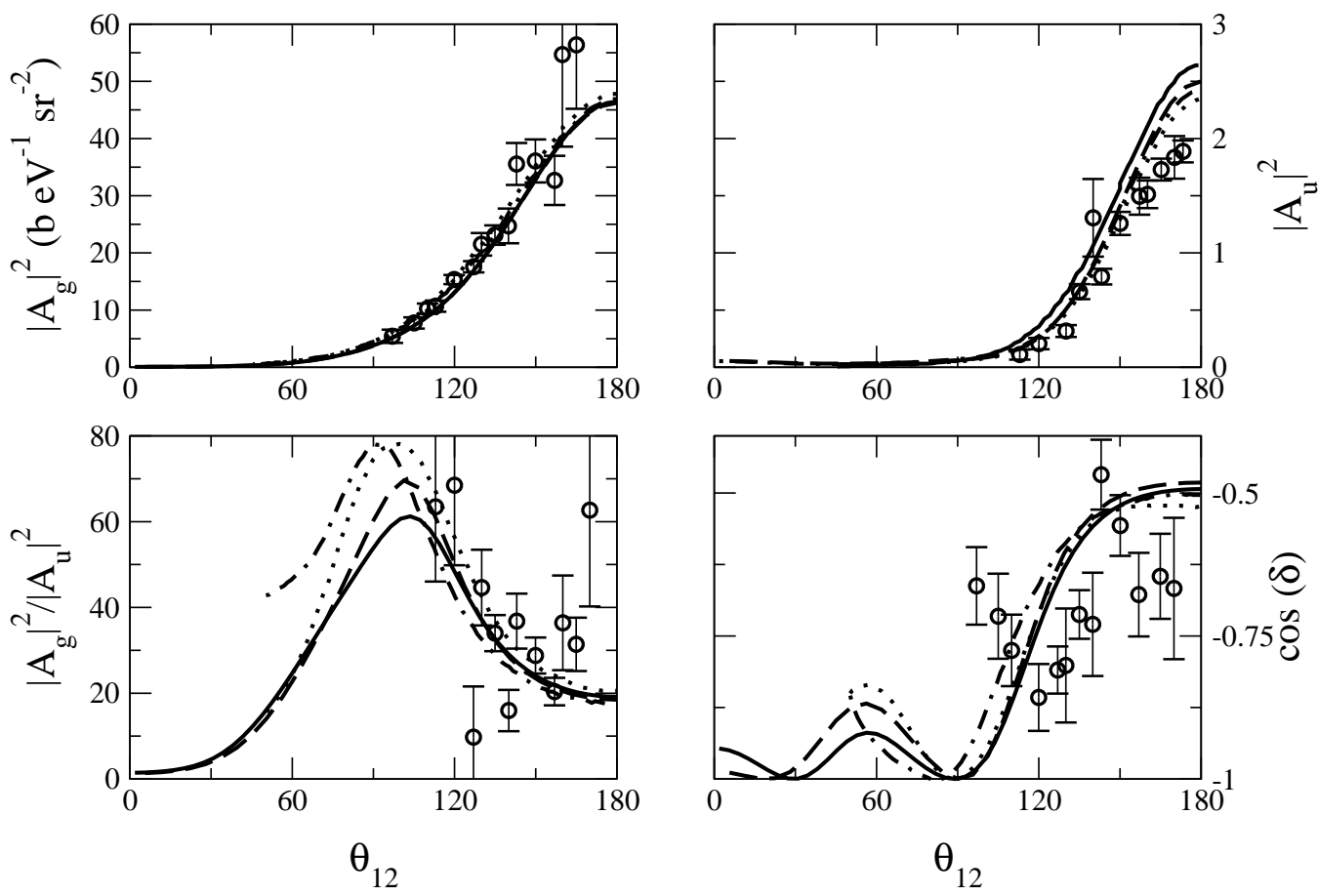

Figure 19. Amplitudes for photon energy $40 \mathrm{eV}$ above threshold with $E_{1}=5 \mathrm{eV}$. Circles: experimental results from [98]. Solid curve: ECS [99], dashed curve: TDCC [99], dotted curve: CCC from [98], dash-dotted curve: HRM-SOW also from [98].

are not sufficient to distinguish the theories. In any case the triple differential cross sections calculated by all of them are generally in good agreement .

\section{Conclusion and outlook}

Theoretical and computational advances over the past few years have made it possible to reduce the simplest (e,2e) and $(\gamma, 2 \mathrm{e})$ problems to practical computation. ECS has played a key role in this development by providing a framework for calculating the exact scattered wave function for the Coulomb three-body system on a finite volume without having to specify its detailed asymptotic form. This approach circumvents the formidable problems posed by trying to numerically implement the formal theory, which to date has not been possible.

At this point, it is logical to ask whether the techniques that have been so successful in solving the Coulomb three-body problem can be extended to look at more complex targets. One can of course treat the problem of electron impact ionization of a multi-electron atom with a simplified frozen-core, single active electron model. At that level of approximation, the problem is reduced to an equivalent 3-body problem and many theoretical studies have been carried out at that level.

The primary motivation for going beyond the frozen-core model is that, even with two active target electrons, there are new ionization processses that present themselves: excitation-ionization, excitation-autoionization and double ionization. In excitation- 
ionization, the atom is singly ionized and the residual ion is left in an excited state. In excitation-autoionization, the target is first excited to an autoionizing state which can then decay into the ionization continuum in a process that competes with direct ionization at the same energy. Double ionization is the (e,3e) process in which there are three free electrons in the final state. To study these kinds of problems, we have to address at minimun a target atom with two active electrons. The ECS method, as originally applied, involves solving large, sparse systems of complex linear equations. Extending this implementation, directly, to a three-electron problem would lead to systems that are extremely large and prohibitivly expensive to solve. To find a viable alternative, it is clearly necessary to seek methods that scale more favorably with the number of electrons.

An implementation of ECS that clearly offers promise in this context is the direct method of Bartlett and Stelbovics, which has now produced highly accurate $(\mathrm{e}, 2 \mathrm{e})$ results for hydrogenic targets [100]. Since their implementation is based on a marching algorithm for computing the scattered wave function, there is good reason to believe that the method can eventually be applied to the four-body Coulomb problem. Time-dependent wavepacket propagation methods also offer considerable promise. Indeed, it has been demonstrated in model calculations by Pindzola, Mitnik and Robicheaux[101] that explicit time propagation methods have scaling properties that allow their application to three-electron systems. In another recent development, we showed that it is possible to combine the idea of time propagation with that of using exterior complex scaling to solve the driven, time-independent Schrödinger equation [71]. The viability of the method was originally demonstrated for a model $3 \mathrm{D}$ breakup process involving only short-range interactions, but we have now used this approach to study e-He ionization in the three-dimensional S-wave model [102].

For the three-body Coulomb problem, the time-independent approaches have the distinct advantage that the analysis of the computed wave function to calculate ionization amplitudes is relatively straightforward. However, with a multi-electron target there are additional complications. The extraction of ionization cross sections from a numerical representation of the scattered wave on a finite grid is substantially more difficult with a multi-electron target than with a one-electron target. If one attempts to compute the ionization cross sections from an integral expression for the breakup amplitudes on a finite grid, one finds that, just as in the e- $\mathrm{H}$ case, the results can be poisoned by the presence of discrete two-body channel terms in the scattered wave. However, in contrast to the e- $\mathrm{H}$ case, the simple expediant of using Coulomb distorted waves does not eliminate the problem, since there is no orthogonality relationship between such continuum functions and the bound states of a multi-electron target. One partial, and not entirely satisfactory, remedy for this problem is what we refer to as "asymptotic subtraction" [71, 103], in which elastic and discrete excitation scattering amplitudes are computed and used to remove two-body terms from the scattered wave. This technique was used in computing differential ionization cross sections for the S-wave e-He problem [102].

For collisional ionization of multi-electron atoms, there are still many details to be worked out and there are still open questions about what will ultimately prove to be the best way to extract ionization cross sections from the wave functions once they are available. Despite the challenges that remain, we are confident that benchmark calculations on the electron-helium system, similar to those that now exist for the electron collisions with hydrogenic targets, will appear in the next few years. 


\section{Acknowledgments}

This work was supported by the U.S. DOE Office of Basic Energy Sciences, Division of Chemical Sciences, and at the University of California Lawrence Berkeley National Laboratory was performed under Contract DE-AC03-76SF00098. CWM gratefully acknowldges a grant from the Ministerio de Educación, Cultura y Deporte (Spain) for a sabbatical research visit to the Universidad Autónoma de Madrid.

\section{References}

[1] Peterkop R K 1962, Opt. Spectrosc. 1387

[2] Rudge M R H and Seaton M J 1965 Proc. Roy. Phys. Soc. 283262

[3] Rudge M R H 1968 Rev. Mod. Phys. 40564

[4] Redmond P J (unpublished), quoted in Rosenberg L 1973 Phys. Rev D 81833

[5] Brauner M, Briggs J S and Klar H 1989 J. Phys. B: At. Mol. Opt. Phys. 222265

[6] Lucey S P, Rasch J and Whelan C T 1999 Proc. Roy. Soc. Lond. A 455349

[7] Jones S and Madison D 2000 Phys. Rev. A 62042701

[8] Kim Y E and Zubarev A L 1997 Phys. Rev. A 56521

[9] Alt E O and Mukhamedzhanov A M 1993 Phys. Rev. A 472004

[10] Kadyrov A S, Mukhamedzhanov A M and Stelbovics A T 2003 Phys. Rev. A 67024702

[11] Mukhamedzhanov A M and Lieber M 1996 Phys. Rev. A 543078

[12] Malegat L, Selles P and Kazansky A K 2000 Phys. Rev. Lett. 854450

[13] Pindzola M S and Schultz D R 1996 Phys. Rev. A $\mathbf{5 3} 1525$

[14] Pindzola M S and Robicheaux F 1996 Phys. Rev. A 542142

[15] Burke P G, Gallaher D F and Geltman S 1969 J. Phys. B: At. Mol. Phys. 21142

[16] Bray I and Stelbovics A T 1992 Phys. Rev. A 466995

[17] Scott M P, Burke P G, Bartschat K and Bray I 1997 J. Phys. B: At. Mol. Opt. Phys. 30 L309

[18] Konovalov D A, Bray I and McCarthy I E 1994 J. Phys. B: At. Mol. Phys. 22 L413

[19] Bray I and Fursa D V 1996 Phys. Rev. A 542991

[20] Bartschat K and Bray I 1996 Phys. Re. A 54 R1002

[21] Bray I 1997 Phys. Rev. Lett. 784721

[22] Stelbovics A T 1999 Phys. Rev. Lett. 831570

[23] Rescigno T N, McCurdy C W, Isaacs W A and Baertschy M 1999 Phys. Rev. A 603740

[24] Pindzola M S, Mitnik D and Robicheaux F 2000 Phys. Rev. A 62062718

[25] Bray I 2000 Aust. J. Phys 53355

[26] Scott M P, Stitt T, Scott N S and Burke P G 2002 J. Phys. B: At. Mol. Opt. Phys. 35 L323

[27] Regge T 1959 Nuovo Cimento 14951

[28] Regge T 1960 Nuovo Cimento 14951

[29] Bottino A, Longoni A M and Regge T 1962 Nuovo Cimento 23954

[30] Balslev E and Combes J M 1971 Commun. Math. Phys. 22280

[31] Simon B 1972 Commun. Math. Phys. 271

[32] Aguilar J and Combes J M 1971 Commun. Math. Phys. 22269

[33] Doolen G D, Nuttal J and Stagat R W 1974 Phys.Rev. A 101612

[34] Reinhardt W 1982 Ann. Rev. Phys. Chem. 33223

[35] Ho Y K 1991 Z. Phys. D 21 191-196

[36] Ho Y K 1991 Phys. Rev. A 44 4154-4161

[37] Nuttall J and Cohen H L 1969 Phys. Rev. 1881542

[38] Simon B 1979 Phys. Lett. A $\mathbf{7 1} 211$

[39] Nicolaides C A and Beck D R 1978 Phys. Lett. A 6511

[40] Rescigno T N, Baertschy M, Byrum D and McCurdy C W 1997 Phys. Rev. A 554253

[41] Kurasov P B, Scrinzi A and Elander N 1994 Phys. Rev. A 495095

[42] Rescigno T N and McCurdy C W 2000 Phys. Rev. A 62032706

[43] Telnov D A and Chu S I 1999 Phys. Rev. A 59 2864-2874

[44] Rom N, Engdahl E and Moiseyev N 1990 J. Chem. Phys. 933413

[45] Elander N and Yarevsky E 1998 Phys. Rev. A $\mathbf{5 7} 3119$

[46] Karlsson H O 1998 J. Chem. Phys. 1083849

[47] Newton R G 1982 Scattering Theory of Waves and Particles Springer-Verlag New York

[48] McCurdy C W and Martín F 2004 J. Phys. B: At. Mol. Opt. Phys. 37917 
[49] Abramowitz M and Stegun I A 1964 Handbook of Mathematical Functions National Bureau of Standards, U. S. Government Printing Office Washington, D. C. p. 878

[50] Baertschy M, Rescigno T N, Isaacs W A and McCurdy C W 1999 Phys. Rev. A 60 R13

[51] Rescigno T N, Baertschy M, Isaacs W A and McCurdy C W 1999 Science 2862474

[52] Baertschy M, Rescigno T N, Isaacs W A, Li X and McCurdy C W 2001 Phys. Rev. A 63 022712

[53] Lill J V, Parker G A and Light J C 1982 Chem. Phys. Lett. 89483

[54] McCurdy C W, Rescigno T N and Byrum D 1997 Phys. Rev. A 561958

[55] McCurdy C W and Rescigno T N 1997 Phys. Rev. A 56 R4369

[56] Manolopoulos D E and Wyatt R E 1988 Chem. Phys. Lett. 15223

[57] Jackson J D 1975 Classical Electrodynamics John Wiley and Sons New York p. 110ff

[58] McCurdy C W, Horner D A, Rescigno T N and Martín F 2004 Phys. Rev. A 69032707

[59] de Boor C 1978 A Practical Guide to Splines Springer, New York

[60] Bachau H, Cormier E, Decleva P, Hansen J E and Martín F 2001 Reports on Progress in Physics 64 1815-1942

[61] Turner J and McCurdy C W 1982 Chem. Phys. 71127

[62] Bartlett P L and Stelbovics A T 2004 Phys. Rev. A 69022703

[63] Bartlett P L and Stelbovics A T 2004 Phys. Rev. A 69040701

[64] Bartlett P L, Stelbovics A T and Bray I 2004 J. Phys. B: At. Mol. Opt. Phys 37 L69

[65] Poet R 1980 J. Phys B: At. Mol. Phys. 132995

[66] Jones S and Stelbovics A T 2002 Phys. Rev. A 66032717

[67] McCurdy C W and Rescigno T N 2000 Phys. Rev. A 62032712

[68] McCurdy C W, Horner D A and Rescigno T N 2001 Phys. Rev. A 63022711

[69] Baertschy M, Rescigno T N, McCurdy C W, Colgan J and Pindzola M S 2001 Phys. Rev. A 63050701

[70] Baertschy M, Rescigno T N and McCurdy C W 2001 Phys. Rev. A 64022709

[71] McCurdy C W, Horner D A and Rescigno T N 2002 Phys. Rev. A 65042714

[72] Rescigno T N, Baertschy M and McCurdy C W 2003 Phys. Rev. A 68020701

[73] Edmonds A R 1957 Angular Momentum in Quantum Mechanics Princeton University Press Princeton p. $37 \mathrm{ff}$

[74] Kadyrov A S, Mukhamedzhanov A M, Stelbovics A T, Bray I and Pirlepesov F 2003 Phys. Rev. A $\mathbf{6 8} 060704$

[75] Bartlett P L, Bray I, Jones S, Stelbovics A T, Kadyrov A S, Bartschat K, Steeg G L V, Scott M P and Burke P G 2003 Phys. Rev. A 68020702

[76] Copson E T 1967 Asymptotic Expansions Cambridge University Press Cambridge, England

[77] Isaacs W A, Baertschy M, McCurdy C W and Rescigno T N 2001 Phys. Rev. A 630307204

[78] Jones J, Madison D H and Baertschy M 2003 Phys. Rev. A 67012703

[79] Röder J, Baertschy M and Bray I 2003 Phys. Rev. A 67010702

[80] Childers J G, James K E, Bray I, Baertschy M and Khakoo M 2003 Phys. Rev. A 68022709

[81] Wannier G 1953 Phys. Rev. 90817

[82] Rost J M 1994 Phys. Rev. Lett. 721998

[83] Shyn T W 1992 Phys. Rev. A 452951

[84] Röder J, Rasch J, Jung K, Whelan C T, Ehrhardt H, Allan R and Walters H 1996 Phys. Rev. A $\mathbf{5 3} 225$

[85] Röder J, Ehrhardt H, Pan C, Starace A F, Bray I and Fursa D 1997 Phys. Rev. Lett. 791666

[86] Bray I 2000 J. Phys. B: At. Mol. Phys. 33581

[87] Röder J 1996 Ph.D. thesis University of Kaiserslautern Germany (unpublished)

[88] Pan C and Starace A F 1991 Phys. Rev. Lett. 67185

[89] Drake G W F, Cassar M M and Nistor R A 2002 Phys. Rev. A 65054501

[90] Wehlitz R, Heiser F, Hemmers O, Langer B, Menzel A and Becker U 1991 Phys. Rev. Lett. 673764

[91] J.Colgan, Pindzola M S and Robicheaux F 2001 J. Phys. B: At. Mol. Opt. Phys. 34 L457-L466

[92] Braüning M, Dörner R, Cocke C L, Prior M H, Kriässig B, Kheifets A S, Bray I, BraüningDemian A, Carnes K, Dreuil S, Mergel V, Richard P, Ulrich J and Schmidt-Böcking H 1988 J. Phys. B: At. Mol. Opt. Phys. 315149

[93] Selles P, Malegat L and Kazansky A K 2002 Phys. Rev. A 65032711

[94] Bolognesi P, Camillion R, Coreo M, Turri G, Berakdar J, Kheifets A S and Avaldi L $2001 \mathrm{~J}$. Phys. B: At. Mol. Opt. Phys. 343193

[95] Malegat L, Selles P and Huetz A 1997 J. Phys. B: At. Mol. Opt. Phys. 30251

[96] Malegat L, Selles P, Lablanquie P, Mazeau J and Huetz A 1997 J. Phys. B: At. Mol. Opt. Phys. 30263 
[97] Kono A and Hattori S 1984 Phys. Rev. A 292981

[98] Bolognesi P, Kheifets A S, Bray I, Malegat L, Selles P, Kazansky A K and Avaldi L $2003 \mathrm{~J}$. Phys. B: At. Mol. and Opt. Phys. 36 L241

[99] Horner D A, Colgan J, Martín F, McCurdy C W, Pindzola M S and Rescigno T N 2004 Phys. Rev. A XX yyyy submitted

[100] Bartlett P L and Stelbovics A T 2004 Phys. Rev. A 69040701

[101] Pindzola M S, Mitnik D and Robicheaux F 1999 Phys. Rev. A 59 4390-4398

[102] Horner D A, McCurdy C W and Rescigno T N 2004 Phys. Rev. A XX xxxx

[103] Rescigno T N and McCurdy C W In Many-Particle Quantum Dynamics in Atomic and Molecular Fragmentation, Ullrich J and Shevelko V P, editors Springer Berlin 2003 